\title{
Stellar Migration and Chemical Enrichment in the Milky Way Disc: A Hybrid Model
}

\author{
James W. Johnson, ${ }^{1 \star}$ David H. Weinberg, ${ }^{1,2,3}$ Fiorenzo Vincenzo, ${ }^{1,2}$ Jonathan C. Bird, ${ }^{4}$ \\ Sarah R. Loebman, ${ }^{5}$ Alyson M. Brooks, ${ }^{6}$ Thomas R. Quinn, ${ }^{7}$ Charlotte R. Christensen, ${ }^{8}$ \\ and Emily J. Griffith ${ }^{1}$ \\ ${ }^{1}$ Department of Astronomy, The Ohio State University, 140 W. 18th Ave., Columbus, OH, 43210, USA \\ 2 Center for Cosmology and Astroparticle Physics (CCAPP), The Ohio State University, 191 W. Woodruff Ave., Columbus, OH, 43210, USA \\ ${ }^{3}$ Institute for Advanced Study, 1 Einstein Dr., Princeton, NJ, 08540, USA \\ ${ }^{4}$ Department of Physics \& Astronomy, Vanderbilt University, 2301 Vanderbilt Place, Nashville, TN, 37235, USA \\ ${ }^{5}$ Department of Physics, University of California Merced, 5200 North Lake Rd., Merced, CA, 95343, USA \\ ${ }^{6}$ Department of Physics \& Astronomy, Rutgers University, 136 Frelinghuysen Rd., Piscataway, NJ, 08854, USA \\ ${ }^{7}$ Department of Astronomy, University of Washington, Box 351580, Seattle, WA, 98195, USA \\ ${ }^{8}$ Department of Physics, Grinnell College, 1116 8th Ave., Grinnell, IA, 50112, USA
}

Accepted XXX; Received YYY; in original form ZZZ

\begin{abstract}
We develop a hybrid model of galactic chemical evolution that combines a multi-ring computation of chemical enrichment with a prescription for stellar migration and the vertical distribution of stellar populations informed by a cosmological hydrodynamic disc galaxy simulation. Our fiducial model adopts empirically motivated forms of the star formation law and star formation history, with a gradient in outflow mass loading tuned to reproduce the observed metallicity gradient. With this approach, the model reproduces many of the striking qualitative features of the Milky Way disc's abundance structure: (i) the dependence of the $[\mathrm{O} / \mathrm{Fe}]-[\mathrm{Fe} / \mathrm{H}]$ distribution on radius $R_{\text {gal }}$ and midplane distance $|z|$; (ii) the changing shapes of the $[\mathrm{O} / \mathrm{H}]$ and $[\mathrm{Fe} / \mathrm{H}]$ distributions with $R_{\mathrm{gal}}$ and $|z|$; (iii) a broad distribution of [O/Fe] at sub-solar metallicity and changes in the [O/Fe] distribution with $R_{\text {gal }},|z|$, and $[\mathrm{Fe} / \mathrm{H}]$; (iv) a tight correlation between $[\mathrm{O} / \mathrm{Fe}]$ and stellar age for $[\mathrm{O} / \mathrm{Fe}]>0.1$; (v) a population of young and intermediate-age $\alpha$-enhanced stars caused by migration-induced variability in the Type Ia supernova rate; (vi) non-monotonic age $-[\mathrm{O} / \mathrm{H}]$ and age- $[\mathrm{Fe} / \mathrm{H}]$ relations, with large scatter and a median age of $\sim 4 \mathrm{Gyr}$ near solar metallicity. Observationally motivated models with an enhanced star formation rate $\sim 2 \mathrm{Gyr}$ ago improve agreement with the observed age-[Fe/H] and age$[\mathrm{O} / \mathrm{H}]$ relations, but worsen agreement with the observed age- $[\mathrm{O} / \mathrm{Fe}]$ relation. None of our models predict an $[\mathrm{O} / \mathrm{Fe}]$ distribution with the distinct bimodality seen in the observations, suggesting that more dramatic evolutionary pathways are required. All code and tables used for our models are publicly available through the Versatile Integrator for Chemical Evolution(VICE; https://pypi.org/project/vice).
\end{abstract}

Key words: methods: numerical - galaxies: abundances, evolution, star formation, stellar content

\section{INTRODUCTION}

The orbits of stars are not fixed. The considerable intrinsic scatter in age-abundance relations of local disc stars (Edvardsson et al. 1993) and the high metallicity of the sun relative to nearby stars of similar age (Wielen, Fuchs \& Dettbarn 1996) provided early evidence that stars in the Milky Way disc can migrate several kiloparsecs from the Galactocentric radius at which they formed. Interest in radial migration as an important element of galactic chemical evolution (GCE) grew further with the demonstration by Sellwood \& Binney (2002) that resonant interactions with transient spiral perturbations could change stars' orbital guiding centre radii without increasing orbital eccentricity, and with subsequent studies showing ubiquitous radial migration in numerical simulations of disc galaxies (e.g. Roškar et al. 2008a,b; Loebman et al. 2011; Minchev et al. 2011; Bird, Kazantzidis \& Weinberg 2012; Bird et al. 2013; Grand, Kawata \& Cropper 2012a,b; Kubryk, Prantzos \& Athanassoula 2013). Schönrich \& Binney (2009a,b) developed the first detailed GCE models incorporating radial migration, describing it with a flexible, dynamically motivated parameterization constrained simultaneously with other GCE

^ Contanct e-mail: johnson.7419@osu.edu parameters when fitting to observations. A number of subsequent studies have incorporated radial migration using similar analytic or parameterized models (e.g. Bilitewski \& Schönrich 2012; Hayden et al. 2015; Kubryk, Prantzos \& Athanassoula 2015a,b; Feuillet et al. 2018; Sharma, Hayden \& Bland-Hawthorn 2020), and Frankel et al. $(2018,2019,2020)$ have used stellar abundances, ages, and kinematics to constrain radial migration empirically.

In this paper we construct evolutionary models for the Milky Way disc that combine a classic multi-ring GCE approach (e.g., Matteucci \& Francois 1989; Wyse \& Silk 1989; Prantzos \& Aubert 1995) with stellar migration predicted by a hydrodynamic simulation of disc galaxy formation from cosmological initial conditions. Our methodology is similar to that of Minchev, Chiappini \& Martig (2013, 2014) and has similar motivations. The use of a cosmological simulation that agrees with many observed properties of the Milky Way assures that our stellar migration scenario is physically plausible, including any correlations of migration in time and space that might be difficult to capture in a parameterized description. Most hydrodynamic cosmological simulations include metal enrichment, and direct comparison between the predicted and observed abundance patterns can provide valuable insights into the accuracy of the simulations and the possible origin of the observed element structure (e.g., Mackereth 
et al. 2017; Grand et al. 2018; Buck 2020; Vincenzo \& Kobayashi 2020; Buck et al. 2021).

However, many ingredients of the simulations' enrichment recipes are uncertain, and metal transport and mixing within the interstellar medium (ISM) are sensitive to numerical resolution and to details of the hydrodynamics and star formation algorithms. Our hybrid approach allows us to consider many choices of uncertain GCE parameters, tuning them to reproduce some observations while leaving others as independent empirical tests. This flexible approach also allows us to isolate the impact of different GCE model ingredients and to zero-in on the ways that stellar migration influences the predicted chemical evolution. In exchange for this exploratory freedom, the hybrid model is not fully self-consistent, instead adopting its own accretion, star formation, and outflow histories rather than the simulation's. Although our methodology can be applied with any given choice of cosmological simulation, we make use of only one in the present paper. One could however apply the same method to multiple simulations to predict a statistical distribution of outcomes.

We focus our predictions and observational comparisons on oxygen, a representative $\alpha$-element producted almost exclusively by core collapse supernovae (CCSN), and iron, which at solar abundances has roughly equal contributions from CCSN and Type Ia supernovae (SN Ia). We will consider other elements with other nucleosynthetic sources in future work, but observed trends of $[\mathrm{Fe} / \mathrm{H}]$ and $[\alpha / \mathrm{Fe}]^{1}$ in the Milky Way disc already display a number of striking features, including:

- At sub-solar $[\mathrm{Fe} / \mathrm{H}]$, the distribution of $[\alpha / \mathrm{Fe}]$ is bimodal, with "high- $\alpha$ " and "low- $\alpha$ " sequences typically separated by $0.1-$ 0.4 dex (e.g., Fuhrmann 1998; Bensby, Feltzing \& Lundström 2003; Adibekyan et al. 2012; Vincenzo et al. 2021a).

- The location of the high- $\alpha$ and low- $\alpha$ sequences is nearly independent of position in the disc, but the relative number of stars in these sequences and the distributions of those stars in $[\mathrm{Fe} / \mathrm{H}]$ changes systematically with Galactocentric radius $R_{\text {gal }}$ and midplane distance $|z|$ (Nidever et al. 2014; Hayden et al. 2015; Weinberg et al. 2019).

- In addition to an overall radial gradient, the shape of the $[\mathrm{Fe} / \mathrm{H}]$ distribution for stars with $|z|<0.5 \mathrm{kpc}$ changes from negatively skewed in the inner disc to roughly symmetric at the solar neighbourhood to positively skewed in the outer disc (Hayden et al. 2015; Weinberg et al. 2019).

- With increasing $|z|$, [Fe/H] distributions become more symmetric and less dependent on $R_{\text {gal }}$ (Hayden et al. 2015).

- The age-metallicity relation (AMR) is broad, with a wide range of $[\mathrm{Fe} / \mathrm{H}]$ at fixed stellar age and vice versa in the solar neighbourhood (Edvardsson et al. 1993) and beyond (Feuillet et al. 2019). The trend of median age with $[\mathrm{Fe} / \mathrm{H}]$ or $[\mathrm{O} / \mathrm{H}]$ is non-monotonic, with solar metallicity stars being younger on average than both metal-poor and metal-rich stars (Feuillet et al. 2018, 2019; Lu et al. 2021).

- The trend of stellar age with $[\alpha / \mathrm{Fe}]$ is much tighter than the trend with $[\mathrm{Fe} / \mathrm{H}]$, becoming broad near $[\alpha / \mathrm{Fe}] \approx 0$ (Feuillet et al. 2018, 2019). Although most stars with $[\alpha / \mathrm{Fe}] \geq 0.1$ are old, observations have revealed a significant population of $\alpha$-rich stars that appear to be young or intermediate age (Chiappini et al. 2015; Martig et al. 2015, 2016; Warfield et al. 2021). Some of these stars may have been "rejuvinated" by stellar mergers or mass-transfer events (Izzard et al. 2018; Silva Aguirre et al. 2018), and the question of what fraction are truly much younger than the median age- $[\alpha / \mathrm{Fe}]$ relation remains open (Hekker \& Johnson 2019).

Many of these results have emerged most clearly from the Apache Point Observatory Galaxy Evolution Experiment (APOGEE; Majewski et al. 2017) of the Sloan Digital Sky Survey (SDSS-III: Eisenstein

1 We follow standard notation where $[X / Y]=\log _{10}(X / Y)-\log _{10}(X / Y)_{\odot}$. Different observational studies use different $\alpha$-elements (or combinations thereof) in abundance ratios, and we will generally use $[\mathrm{O} / \mathrm{Fe}]$ and $[\alpha / \mathrm{Fe}]$ synonymously. et al. 2011; SDSS-IV: Blanton et al. 2017), sometimes confirming and extending trends suggested by earlier observational data. We will assess the degree to which models with fairly conventional GCE assumptions coupled to simulation-based radial and vertical migration of stars can explain, or fail to explain, these observations.

Relative to Minchev et al. (2013, 2014), our base GCE model has many differences of detail, the most important being our inclusion of outflows, which is in turn connected to our different choice of oxygen and iron yields. Our simulation, the galaxy h277 from the Christensen et al. (2012) suite evolved with the N-body+SPH code GASOLINE (Wadsley et al. 2004), is fully cosmological, while the simulation used by Minchev et al. $(2013,2014)$ has a more idealized geometry with merger and accretion history drawn from a larger cosmological volume (Martig et al. 2012). The Minchev et al. (2013, 2014) simulation has a fairly strong, long-lived bar while h277 has only a weak, transient bar, and this difference could have some impact on radial migration. Another methodological difference, which turns out to be important for some observables, is that we track enrichment from stellar populations as they migrate (see $\S 3.4$ below), while Minchev et al. $(2013,2014)$ assume that populations enrich only the radial zone in which they were born.

Previous studies have shown that h277 and other disc galaxies evolved with similar physics have realistic rotation curves (Governato et al. 2012; Christensen et al. 2014a,b), stellar mass (Munshi et al. 2013), metallicity (Christensen et al. 2016), dwarf satellite populations (Zolotov et al. 2012; Brooks \& Zolotov 2014), and HI properties (Brooks et al. 2017). Most directly relevant to this study, Bird et al. (2020) demonstrate that h277 accurately reproduces the observed relation between stellar age and vertical velocity dispersion $\sigma_{z}$. This relation arises as a consequence of "upside-down" disc formation in which the star-forming gas layer becomes thinner with time as well as the dynamical heating of stars as they age (Bournaud \& Elmegreen 2009; Bournaud, Elmegreen \& Martig 2009; Forbes, Krumholz \& Burkert 2012; Bird et al. 2013; Vincenzo, Kobayashi \& Yuan 2019; Yu et al. 2021). Schönrich \& Binney (2009a) distinguish between the radial mixing caused by "blurring" of stars on moderately eccentric orbits and "churning" that changes the guiding centre radii of their orbits. Both phenomena occur in our simulation and we do not attempt to separate them, simply using the terms "migration" or "mixing" to refer to the combined effect. In addition to radial migration, we use the $\mathrm{h} 277$ predictions for the vertical locations (i.e., midplane distances $|z|$ ) of stars at the present day. Our GCE model assumes that the gas disc is vertically well mixed, so a stellar population's birth abundances depend only on $R_{\text {gal }}$ and time. Vertical gradients arise because older populations have larger $\sigma_{z}$ and thus larger average $|z|$, and also because radial migration is coupled to changes in $\sigma_{z}$ (Solway, Sellwood \& Schönrich 2012). The good match to the observed age-velocity relation found by Bird et al. (2020) allows us to use vertical trends of abundance ditributions as a further test of our chemical evolution model.

We describe the h277simulation further in $\S 2.1$ and our implementation of radial migration in $\S 2.2$. We describe the base GCE model in $\S \S 2.5-2.8$. Distinctive features of our GCE model are the use of a radially dependent outflow mass loading $\eta\left(R_{\text {gal }}\right)$ to tune the metallicity gradient, our implementation of a star formation law motivated by spatially resolved studies of nearby galaxies and highredshift studies of its time-dependence, and our use of mean radial age trends of disc galaxies to set the radial dependence of the star formation history (SFH). Our fiducial model adopts a smooth SFH with an "inside-out" radial trend in which star formation proceeds more rapidly in the inner Galaxy. Motivated by the observational analyses of Isern (2019) and Mor et al. (2019), we also consider models with a burst of star formation centred $\approx 2 \mathrm{Gyr}$ in the past, similar to the one-zone models investigated by Johnson \& Weinberg (2020). Other authors have suggested multiple bursts in the Milky Way's SFH (e.g., Lian et al., 2020a,b; Ruiz-Lara et al. 2020; Sysoliatina \& Just 2021), perhaps triggered by satellite interactions, while others have advocated a two-phase SFH to explain the $[\alpha / \mathrm{Fe}]$ dichotomy (e.g., Chiappini, Matteucci \& Gratton 1997; Haywood et al. 2016; Spitoni 
et al. 2019; Buck et al. 2020; Khoperskov et al. 2021). We do not investigate these more complex SFHs here, but we plan to do so in future work.

\section{METHODS}

To fulfill the goals of this paper, we develop and make use of newly released features within the Versatile Integrator for Chemical Evolution (VICE; Johnson \& Weinberg 2020), an opensource python package available for Unix system architectures (for further details, see Appendix C). These features are designed to handle models such as these with a wide range of flexibility. We reserve a description of VICE's migration algorithm and our simulation parameters for $\S 2.2$, first describing our sample of star particles from the hydrodynamic simulation.

Although we make use of a hydrodynamic simulation to drive stellar migration, we do not make use of its SFH. While our study employs similar techniques as Minchev et al. (2013), ours differs slightly in that they take a single SFH which is similar, but not identical to that of their N-body+SPH simulation; in this paper we present a handful of assumptions about the SFH, which do not necessarily resemble that of $h 277$. An alternative to modeling radial migration based on a hydrodynamical simulation is to invoke dynamical arguments; Schönrich \& Binney (2009a) and Sharma et al. (2020) take such an approach. This method, however, introduces free parameters which then require fitting to data. An advantage of our technique is that there are no free parameters describing radial migration introduced to the model; it is unclear how much fitting radial migration parameters could bias the model into agreement with parts of the data not involved in the fitting process. Instead we adopt a physically motivated migration model evolved from cosmological initial conditions. We rely on a single realization of this numerical model and are thus subject to the differences between the dynamical history of this simulated galaxy and that of the Milky Way. However, one could compare the predictions made by our chemical evolution models when applied to different hydrodynamical simulations, a direction we plan to pursue in future work. We emphasize that the hydrodynamical simulation only informs the mixing processes in our models, and there is no N-body integration involved in our models aside from that which was used to run the h277 simulation in the first place.

\subsection{The Hydrodynamical Simulation}

In this paper we make use of star particles from the h277 simulation (Christensen et al. 2012; Zolotov et al. 2012; Loebman et al. 2012, 2014; Brooks \& Zolotov 2014). Recently employed to study the stellar age-velocity relationship, a synopsis of its detailed simulation parameters and cosmological model can be found in $\S 2$ of Bird et al. (2020). We do not repeat these details here, instead focusing on how we vet the sample of star particles for use in our chemical evolution models.

The parameters of stars that we use in our analysis are the birth and final radii and the final midplane distance. $\mathrm{h} 277$ did not record the exact birth radius of each star particle; however, each star particle does have an accurate age at each snapshot. The orbital radii of stars that are sufficiently young in their first snapshot should be good approximations of their birth radii. We therefore restrict our sample to those star particles with an age at first snapshot of $\leq 150 \mathrm{Myr}$, and we adopt their Galactocentric radius at that time as their birth radius. We have repeated our analysis with a maximum age at first snapshot of $50 \mathrm{Myr}$ and found similar results, indicating that these time intervals are short enough to not impact our conclusions. We adopt the $150 \mathrm{Myr}$ interval because it provides a larger number of star particles to sample from. Of the star particles that remain after imposing this cut, the oldest has an age of $13.23 \mathrm{Gyr}$ at the present day (i.e. at the simulation's final output). Our GCE model can only apply on timescales as long as or shorter than the full range of ages of the sample of star particles; we therefore subtract $0.5 \mathrm{Gyr}$ from the formation times of all star particles, allowing $T=0$ in our models to correspond to $T=0.5 \mathrm{Gyr}$ in $\mathrm{h} 277$. As a consequence, our disc models trace the chemical evolution of the Galaxy out to a lookback time of $\sim 13.2 \mathrm{Gyr}$, or a redshift of $z \approx 9$, placing the onset of star formation at that time.

We further restrict our sample of star particles to only those with both formation and final radii of $R_{\mathrm{gal}} \leq 20 \mathrm{kpc}$, and to have formed within $|z| \leq 3 \mathrm{kpc}$ of the disc midplane. These criteria are intended to restrict our sample to star particles that formed within the disc and can therefore be described by a disc GCE model. While it is possible that some star particles formed in a dwarf galaxy happen to satisfy our geometrical cuts at the star's formation time, these particles are few in number, and are only relevant at large $R_{\text {gal }}$ and high ages: a region of parameter space where few stars form anyway.

Based on a kinematic decomposition performed on the presentday phase space distribution of the h277 star particles conducted with the analysis.decomp routine within the pynbody package (Pontzen et al. 2013), we classify each star particle as having thin disc, thick disc, bulge, pseudobulge, or halo-like kinematics. Details on the decomposition process can be found in Brook et al. (2012) and Bird et al. (2013). We include the entire sample in VICE's public code base, but only make use of those with disc-like kinematics in this paper. Our geometric selection yields 3,152,211 star particles in total, 1,751,765 of which have disc-like kinematics and are included in our sample.

h277 had a weak and transient bar during its evolution, but it does not have one at $z=0$. This is a noteworthy difference between our model and that of Minchev et al. (2013), and by extension the Minchev et al. $(2014,2017)$ studies as well, because they selected a hydrodynamic simulation of a galaxy specifically so that it would have a strong bar at $z=0$. This could mean that the dynamical history of our model Galaxy differs from that of Minchev et al. (2013) and perhaps the Milky Way itself. However, the difference is likely within the uncertainties of the current understanding of the Milky Way's dynamical history. Although an investigation of the impact of bar evolution on stellar migration and thus chemical evolution is outside the scope of this paper, it is an interesting question that can be probed by simply swapping the h277 data within VICE for another simulation, then rerunning our numerical models and comparing the results.

In the top row of Fig. 1, we plot the distributions of final radius in bins of birth radius and age for our sample of star particles. Conversely, the bottom row shows distributions of birth radii in bins of final radius and age. Focusing on the top row of panels, we note that for star particles born at any radius and time, the distribution of final radius is still peaked near the birth radius, but the peak moves slightly inward with increasing age. The tails of the distributions toward larger $R_{\text {gal }}$ are nearly age-independent, while the tails toward smaller $R_{\text {gal }}$ are not. This suggests that radial migration inward and outward occur on different timescales in h277, specifically that inward migration is slower than outward migration. By extension, this suggests that the two may be tied to different physical processes. Alternatively, it may simply be that stars that migrate to the outer Galaxy are no longer subject to strong dynamical perturbations while stars that move inward can still experience strong orbital disturbances.

Focusing on the bottom row of panels in Fig. 1, we note that the modes of the birth radius distributions show a much stronger dependence on age than the modes of the final radius distributions. At any Galactocentric radius at the present day, the youngest stars are overwhelmingly born at comparable radii, while the oldest stars are overwhelmingly born at smaller radii. This trend is most noticeable at large $R_{\mathrm{gal}}$. The differences between the final radius and birth radius distributions can be understood by considering the radial gradient of stellar surface density: there are more stars at small radius to move outward than vice versa, so roughly symmetric evolution of $R_{\text {final }}$ produces strongly asymmetric evolution of $R_{\text {birth }}$.

Taking $\left|\Delta R_{\text {gal }}\right| \geq 500$ pc between birth and final radii as the criterion for migration inward or outward, we find as global percentages in our sample that $27 \%$ of star particles migrated inward, $29 \%$ migrated outward, and the remaining $44 \%$ stayed near their birth radius. 


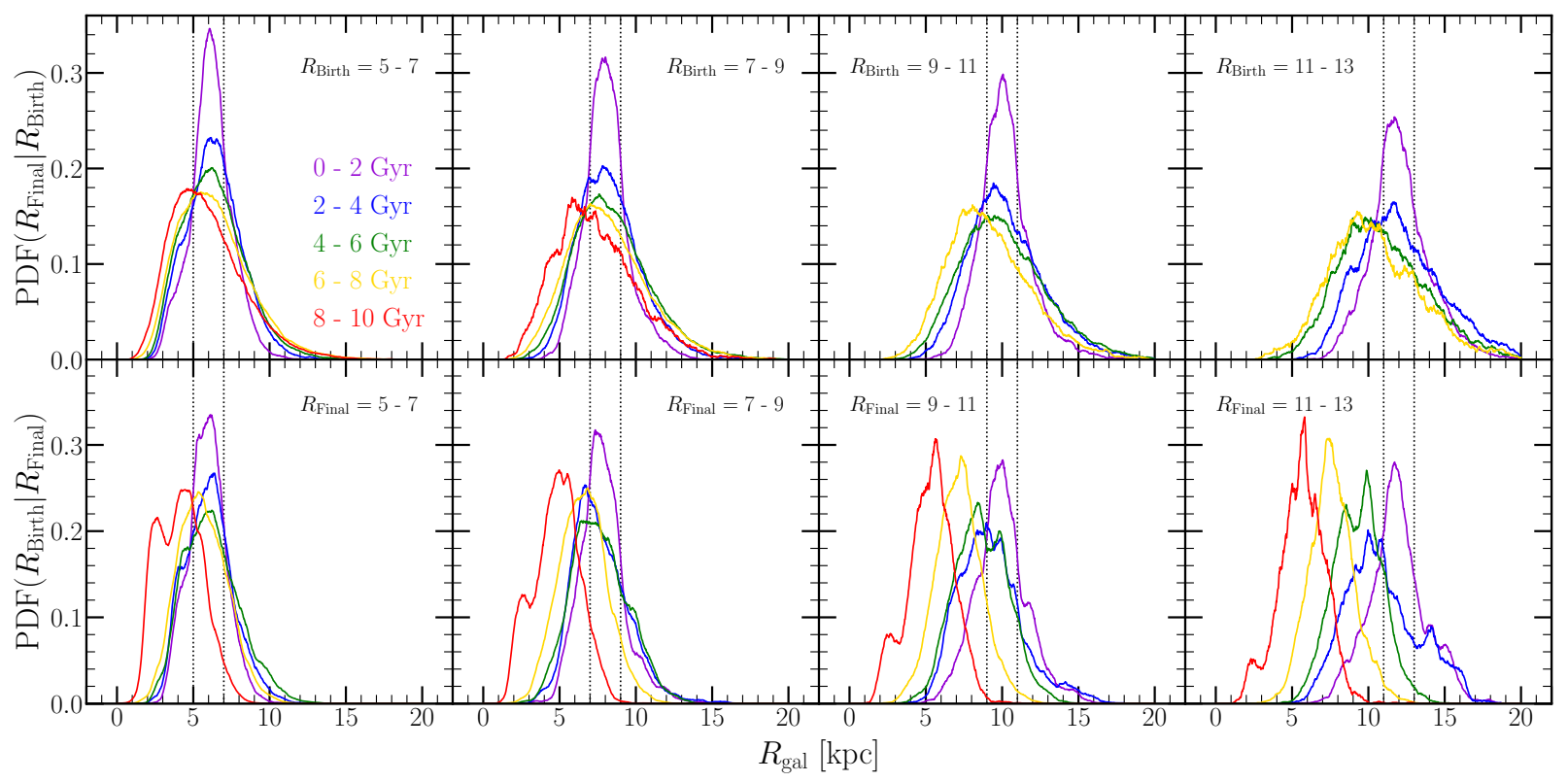

Figure 1. Radial distributions of our sample of star particles from h277. In the top row, we show distributions of final radius in bins of birth radius and age, and in the bottom row, we show distributions of birth radius in bins of final radius and age. Each bin in Galactocentric radius is shown in its own panel, denoted in text at the top of each panel and by vertical black dashed lines. We colour-code the distributions according to the age of the star particles, denoted by the legend in the upper left panel. We smooth all distributions with a box-car width of $0.5 \mathrm{kpc}$ to improve visual clarity. We omit the distributions for 8 - 10 Gyr old stars born in the 9 - 11 and $11-13 \mathrm{kpc}$ bins due to an insufficient number of star particles with which to calculate the distribution.

As one can see from the top panels of Fig. 1, a large fraction of migration away from birth radius has already occurred by the time stars are $\sim 2$ Gyr old. If the SN Ia delay-time distribution (DTD) is a $t^{-1.1} \approx t^{-1}$ power-law as suggested by observational results (e.g. Maoz \& Mannucci 2012; Maoz \& Graur 2017), then we expect similar numbers of SN Ia events to occur with delay times between 0.1 $1 \mathrm{Gyr}$ and $1-10 \mathrm{Gyr}$. With an extended DTD and the timescales for migration implied by Fig. 1, SN Ia progenitors can migrate significant distances before exploding. This effect has largely been neglected by GCE studies to date on the grounds that radial migration is a slow process, and thus the majority of nucleosynthesis should occur near a star's birth radius (e.g. as assumed in Minchev et al. 2013, and the application of the Weinberg, Andrews \& Freudenburg 2017 analytic models in Feuillet et al. 2018). However, we show below that radial migration within the timescale of the SN Ia DTD can have an important impact on some aspects of chemical evolution.

\subsection{Radial Migration}

As in previous studies (e.g. Matteucci \& Francois 1989; Schönrich \& Binney 2009a; Minchev et al. 2013; Sharma et al. 2020), in this paper we model the Milky Way as a series of concentric rings ${ }^{1}$ with a uniform width $\Delta R_{\text {gal }}$. To run numerical simulations of these models, we develop and make use of VICE's milkyway object, designed specifically for such an approach. The milkyway object is a subclass of a more general object named multizone; at its core a multizone object is an array of singlezone objects, which are designed to handle one-zone models of GCE and were the focus of Johnson \& Weinberg (2020), VICE's initial release paper. The multizone object affords users full control over which zone any individual stellar population is in at all timesteps following its formation as well as the ability to move gas between any two zones with any time dependence. In principle this should allow for arbitrarily complex zone configurations and migration prescriptions. The milkyway object is a user-friendly extension of the multizone base class, which enforces an annular zone configuration as we take here. As defaults, it adopts

${ }^{1}$ For clarity, we use the term "ring" to refer to a computational zone of our calculation (100 pc in radial range) and the term "annulus" to refer to a larger radial range (typically $2 \mathrm{kpc}$ ). the stellar migration model detailed in this section, our star formation law discussed in $\S 2.6$, and the scaling of the outflow mass loading factor $\eta$ with radius $R_{\text {gal }}$ parameterized in $\S 2.4$.

As in hydrodynamical simulations, star particles in VICE are standins for entire stellar populations. They are said to be in a given zone if their radius is between the inner and outer edges of the ring. At all times, their nucleosynthetic products and returned envelopes are placed in the ISM of the ring that they are in at that time. VICE forms a fixed number of stellar populations per zone per timestep, and it allows their masses to vary to account for variations in the SFR. The total mass of stars formed in a given zone and timestep is divided evenly among the corresponding stellar populations, which can then experience different stellar migration histories.

The final radius of a stellar population is then determined based on the birth and final radii of star particles in the hydrodynamical simulation. Describing the Galaxy as a series of concentric rings, VICE's milkyway object assumes stellar populations are born at the centres of each ring. For a stellar population born at a time $T$ and Galactocentric radius $R_{\text {gal }}$, it first searches for star particles from $\mathrm{h} 277$ that formed at $T \pm 250 \mathrm{Myr}$ and $R_{\mathrm{gal}} \pm 250 \mathrm{pc}$. It then randomly selects a star particle from this subsample to act as an analogue. This stellar population then adopts the change in orbital radius $\Delta R_{\text {gal }}$ of its analogue, and moves from its birth radius to the implied final radius at $T=13.2 \mathrm{Gyr}$ with an assumed time dependence (see below). If no candidate analogues are found, VICE widens the search to $T \pm 500$ Myr and $R_{\text {gal }} \pm 500 \mathrm{pc}$. If still no analogue is found, then it finds the star particle with the smallest difference in birth radius still within a birth time of $T \pm 500 \mathrm{Myr}$, and assigns it as the analogue. While this prescription allows stellar populations to be assigned analogues with significantly different birth radii, this is only an issue for small $T$ and large $R_{\text {gal }}$ where there are few star particles from $\mathrm{h} 277$, and where few stars form in nature anyway due to the inside-out growth of galaxies (e.g. Bird et al. 2013). Furthermore, due to the similarity of the histograms in the top row of Fig. 1, we expect taking $\Delta R_{\text {gal }}$ from a star particle that formed at a similar time but different birth radius in these instances to be accurate enough for our purposes. When an h277 star particle is assigned as an analogue, it is not thrown out of the sample of candidate analogues, in theory allowing a star particle to act as an analogue for multiple stellar populations. Because these 


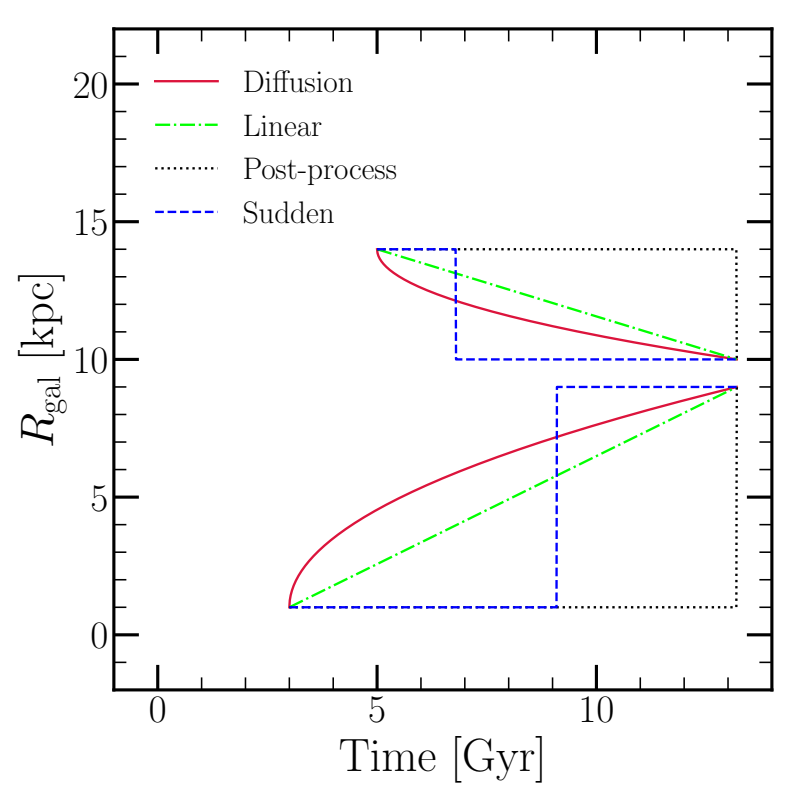

Figure 2. A diagram illustrating how Galactocentric radius changes with time for two stellar populations under our migration schema: diffusion (crimson, solid), linear (lime, dot-dashed), post-process (black, dotted), and sudden (blue, dashed). Here the initial and final radii and birth times are chosen at random for illustrative purposes. With the initial and final Galactocentric radii of a stellar population, its birth time, and one of these assumptions regarding the time-dependence of radial migration, the Galactocentric radius at all times is known. Diffusion is our default model.

populations will have similar $R_{\text {form }}$ and $T_{\text {form }}$, they will have similar but not identical abundances.

We neglect radial gas flows in the present paper (Bilitewski \& Schönrich 2012; Lacey \& Fall 1985), instead focusing our comparisons on the time-dependence with which stars migrate. We consider four models describing the evolution between a star particle's birth and final radius:

- Post-Processing: Stars stay where they are born until the final timestep, at which point they instantly migrate to their final radius. This retains the assumption that stars do not contribute to nucleosynthesis beyond their birth radius as employed in previous studies (e.g. Minchev et al. 2013). In this scenario, the ISM of each ring is treated as a one-zone model independent of all other zones. We illustrate this case with a black dotted line in Fig. 2.

- Sudden: A random number is drawn from a uniform distribution between a stellar population's time of birth and the present day. That time is taken to be the time of instantaneous migration to the presentday annulus. This emulates a scenario in which a single dynamical interaction rapidly changes a star's orbital radius, and it can be thought of mathematically as a generalization of the post-processing scenario. We illustrate this case with a blue dashed line in Fig. 2.

- Diffusion: Stars move to their final radii in a continuous, timedependent manner, with displacement $\propto \sqrt{\text { age. This scenario corre- }}$ sponds to diffusion of angular momentum by a random walk, similar to the assumption used by Frankel et al. $(2018,2020)$. We illustrate this case with a red solid line in Fig. 2.

- Linear: A simple variation of the diffusion model in which the migration to the final radius scales linearly with age rather than with $\sqrt{\text { age }}$. We illustrate this case with a green dot-dashed line in Fig. 2.

The diffusion model has the clearest physical motivation, and it is our default assumption used in all cases unless otherwise noted. The other models provide illustrative contrasts and allow us to investigate our model's sensitivity to the details of radial migration. We do not distinguish between "blurring" and "churning" (Schönrich \& Binney 2009a), terms frequenly used to refer to a star's epicyclic motions and changes in the guiding centre of its orbit, respectively. Both effects, induced by a wide variety of underlying causes such as molecular cloud scattering (Mihalas \& Binney 1981; Jenkins \& Binney 1990;
Jenkins 1992), orbital resonances with spiral arms or bars (Sellwood \& Binney 2002; Minchev et al. 2011), and satellite perturbations (Bird et al. 2012), are present in the h277 simulation.

Our GCE model assumes that the star-forming ISM is vertically and azimuthally mixed within each radial annulus. The abundances assigned to a stellar population depend on its birth radius and time, but they do not depend on its distance from the plane. Nonetheless, as shown below, the abundance patterns of our simulation exhibit clear vertical gradients because older stellar populations have thicker vertical distributions (Bird et al. 2020). Radial migration is also coupled to vertical dynamics in complex ways (Solway et al. 2012; Minchev et al. 2012). We will see that these effects already suffice to explain many of the observed vertical trends of Milky Way disc abundances.

\subsection{Nucleosynthetic Yields}

We focus our analysis on alpha and iron-peak elements, taking oxygen $(\mathrm{O})$ and iron $(\mathrm{Fe})$ as the representative cases. The dominant enrichment channels of interest in our models are thus CCSN and SN Ia (Johnson 2019). We would expect similar results for other alpha (e.g. $\mathrm{Ne}, \mathrm{Mg}, \mathrm{Si}$ ) and iron-peak elements (e.g., $\mathrm{Cr}, \mathrm{Ni}$ ), with quantitative differences reflective of their relative yields. Odd- $Z$ iron-peak elements (e.g., V, Mn, Co) could behave somewhat distinctly because of metallicity-dependent yields.

CCSN enrichment happens immediately following the formation of progenitor stars in VICE. This is an adequate approximation, because the lifetimes of massive stars are short compared to the relevant timescales for galaxy evolution. For the most massive stars, the lifetimes are comparable to the timestep size we adopt in our numerical integrations. This assumption implies a linear relationship between the CCSN enrichment rate and the SFR:

$$
\dot{M}_{x}^{\mathrm{CC}}=y_{x}^{\mathrm{CC}} \dot{M}_{\star}
$$

where $y_{x}^{\mathrm{CC}}$ is the CCSN yield of some element $x$. Physically, this quantity represents the mass of an element $x$ ejected to the ISM from all CCSN events associated with a stellar population in units of the stellar population's initial mass. For example, if $y_{x}^{\mathrm{CC}}=0.01$, a hypothetical $100 \mathrm{M}_{\odot}$ stellar population would add $1 \mathrm{M}_{\odot}$ of $x$ to the ISM immediately. In this paper, we adopt $y_{\mathrm{O}}^{\mathrm{CC}}=0.015$ and $y_{\mathrm{Fe}}^{\mathrm{CC}}=$ 0.0012 from Johnson \& Weinberg (2020), who in turn adopt these values from Weinberg et al. (2017).

SN Ia nucleosynthesis products are injected according to a $t^{-1.1}$ DTD with a minimum delay time of $t_{\mathrm{D}}=150 \mathrm{Myr}$. This is the default DTD in VICE, which was also adopted by Johnson \& Weinberg (2020), and is suggested by recent observational results comparing the cosmic SN Ia rate to the cosmic SFH (Maoz \& Mannucci 2012; Maoz \& Graur 2017). In a one-zone model at times $t>t_{\mathrm{D}}$, the enrichment rate of some element $x$ can be expressed as the product of some yield $y_{x}^{\text {Ia }}$ and the SFH weighted by the DTD:

$$
\begin{aligned}
\dot{M}_{x}^{\mathrm{Ia}} & =y_{x}^{\mathrm{Ia}}\left\langle\dot{M}_{\star}\right\rangle_{\mathrm{Ia}} \\
& =y_{x}^{\mathrm{Ia}} \frac{\int_{0}^{t} \dot{M}_{\star}\left(t^{\prime}\right) R_{\mathrm{Ia}}\left(t-t^{\prime}\right) d t^{\prime}}{\int_{t_{\mathrm{D}}}^{t_{\mathrm{max}}} R_{\mathrm{Ia}}\left(t^{\prime}\right) d t^{\prime}}
\end{aligned}
$$

where $R_{\mathrm{Ia}}$ is the DTD itself, which has units of $M_{\odot}^{-1} \mathrm{Gyr}^{-1}$. Like the CCSN yield, $y_{x}^{\text {Ia }}$ is the mass of some element $x$ produced by SNe Ia over the time interval $t_{\mathrm{D}}-t_{\mathrm{max}}$, in units of the stellar population's initial mass. It can also be expressed as an integral over the DTD:

$y_{x}^{\mathrm{Ia}}=m_{x}^{\mathrm{Ia}} \int_{t_{\mathrm{D}}}^{t_{\mathrm{max}}} R_{\mathrm{Ia}}\left(t^{\prime}\right) d t^{\prime}=m_{x}^{\mathrm{Ia}} \frac{N_{\mathrm{Ia}}}{M_{\star}}$

where $m_{x}^{\text {Ia }}$ is the average mass of the element $x$ produced in a single SN Ia event and the integral evaluates to the mean number of SN Ia events $N_{\text {Ia }}$ per mass of stars formed $M_{\star}$. VICE forces $t_{\max }=15$ Gyr always, though provided one is consistent with equations (2b) and (3), the results are independent of $t_{\max }$ because the integrals 
cancel. Extending this formalism to multi-zone models is simple; rather than an integral over the star formation history of a given annulus, the rate becomes a summation over all stellar populations that are in a given zone at some time:

$\dot{M}_{x}^{\mathrm{Ia}}=y_{x}^{\mathrm{Ia}} \frac{\sum_{i} M_{i} R_{\mathrm{Ia}}\left(\tau_{i}\right)}{\int_{t_{\mathrm{D}}}^{t_{\mathrm{max}}} R_{\mathrm{Ia}}\left(t^{\prime}\right) d t^{\prime}}$

where $M_{i}$ and $\tau_{i}$ are the mass and age of the $i$ 'th stellar population, respectively.

Initially, we adopted $y_{\mathrm{O}}^{\mathrm{Ia}}=0$ and $y_{\mathrm{Fe}}^{\mathrm{Ia}}=0.0017$ from Johnson \& Weinberg (2020), who in turn adopt these values from Weinberg et al. (2017). However, we found that the e-folding timescales of star formation in our models are sufficiently long (see discussion in $\S 2.5$ ) that our fiducial, inside-out $\mathrm{SFH}$ model predicted $[\mathrm{O} / \mathrm{Fe}] \approx+0.05$ for young stars. We therefore multiply $y_{\mathrm{Fe}}^{\mathrm{Ia}}$ by $10^{0.1}$, adopting $y_{\mathrm{Fe}}^{\mathrm{Ia}}=$ 0.00214 so that our fiducial model predicts a late-time $[\mathrm{O} / \mathrm{Fe}]$ ratio in better agreement with observations. Changes at this level are within the uncertainties of SN Ia rates and yields, so we consider it reasonable to adjust the yields empirically to reproduce observed abundances.

Our IMF-averaged O and Fe yields are based on a Kroupa (2001) IMF combined with supernova nucleosynthesis models in which most $M>8 \mathrm{M}_{\odot}$ stars explode as a CCSN (e.g. Chieffi \& Limongi 2004, 2013). Recent studies have strongly suggested that many high mass stars instead collapse directly to a black hole (see theoretical discussion by, e.g., Pejcha \& Thompson 2015; Sukhbold et al. 2016; Ertl et al. 2016, and observational evidence from Gerke, Kochanek \& Stanek 2015; Adams et al. 2017; Basinger et al. 2020). Our yields would be lower in a scenario with extensive black hole formation and/or a steeper high mass IMF (Griffith et al. 2021). These effects could also introduce a metallicity dependence if the landscape of black hole formation changes with metallicity. The strong increase of the specific SN Ia rate seen at low galaxy masses (Brown et al. 2019) provides circumstantial evidence of a higher $R_{\text {Ia }}$ normalization at low metallicity; the stellar close binary fraction also depends on metallicity (Badenes et al. 2018; Moe, Kratter \& Badenes 2019). However, there is presently no solid empirical basis for adopting metallicity dependent $\mathrm{O}$ and $\mathrm{Fe}$ yields over the range relevant to this paper (roughly $-0.8 \leq[\mathrm{Fe} / \mathrm{H}] \leq 0.4$ ), and some empirical evidence that any metallicity trends in this range are weak (Weinberg et al. 2019). VICE has capabilities to compute IMF-averaged yields for a flexible description of the massive star explodability landscape, as described by Griffith et al. (2021) and the VICE documentation, but we do not use this methodology here. We expect that most of our results would be largely unchanged if we were to lower all three yields $\left(y_{\mathrm{O}}^{\mathrm{CC}}, y_{\mathrm{Fe}}^{\mathrm{CC}}, y_{\mathrm{Fe}}^{\mathrm{Ia}}\right)$ by the same factor and adjust our adopted outflow mass loading prescription to compensate (see below).

Both AGB star enrichment and the recycling of previously produced metals in this paper proceed as they did in Johnson \& Weinberg (2020), with the caveat that the mass is added to the ring that a stellar population is in at a given time, which may or may not be the ring it was born in. Recycling proceeds according to the Kalirai et al. (2008) initial-remnant mass relation assuming a Kroupa (2001) IMF and a mass-lifetime relationship of $\tau=1.1 \tau_{\odot}\left(M / M_{\odot}\right)^{-3.5}$, where $\tau_{\odot}=$ $10 \mathrm{Gyr}$ is the main sequence lifetime of the sun and the factor of 1.1 accounts for the post-main sequence lifetime. VICE includes AGB enrichment in all models; here we adopt the net yields sampled on a table of stellar initial mass and metallicity from the FRUITY database (Cristallo et al. 2011). However, the AGB star yields of $\mathrm{O}$ and $\mathrm{Fe}$ are tiny compared to their supernova yields, so they have negligible impact on the results presented in this paper.

\subsection{Outflows}

Weinberg et al. (2017) demonstrate that, to first order, the nucleosynthetic yields of a given element and the strength of outflowing winds determine the late-time equilibrium abundance in the ISM, with a secondary dependence on the SFH. We retain their characterization of outflows here, in terms of a mass-loading factor $\eta$ describing the ratio of the mass outflow to the SFR:

$\eta \equiv \frac{\dot{M}_{\text {out }}}{\dot{M}_{\star}}$

We adopt a scaling of $\eta$ with $R_{\text {gal }}$ such that the late-time equilibrium abundance as a function of radius describes a metallicity gradient in agreement with observations. For a constant SFR, the equilibrium abundance of an $\alpha$-element, produced by CCSNe with a metallicityindependent yield, is given by

$Z_{\alpha, \mathrm{eq}}=\frac{y_{\alpha}^{\mathrm{CC}}}{1+\eta\left(R_{\mathrm{gal}}\right)-r}$,

where $r$ is the recycling parameter $(\approx 0.4$ for the sake of this scaling with a Kroupa 2001 IMF; see discussion in $\S 2.2$ of Weinberg et al. 2017). Solving for $\eta\left(R_{\text {gal }}\right)$ yields

$\eta\left(R_{\mathrm{gal}}\right)=\frac{y_{\alpha}^{\mathrm{CC}}}{Z_{\alpha, \mathrm{eq}}}+r-1=\frac{y_{\alpha}^{\mathrm{CC}}}{Z_{\alpha, \odot}} 10^{-\operatorname{mode}([\alpha / \mathrm{H}])\left(R_{\mathrm{gal}}\right)}+r-1$,

where mode $([\alpha / \mathrm{H}])\left(R_{\text {gal }}\right)$ denotes the mode of the stellar $[\alpha / \mathrm{H}]$ distribution at a radius $R_{\text {gal }}$, which we assume to correspond to the equilibrium abundance at that radius. Recent studies of the disc metallicity gradient with APOGEE find values of $-0.09 \mathrm{dex} / \mathrm{kpc}$ to -0.06 dex/kpc (e.g. Frinchaboy et al. 2013; Hayden et al. 2014; Weinberg et al. 2019), consistent with earlier studies. Here we adopt a slope of $-0.08 \mathrm{dex} / \mathrm{kpc}$ and set $\operatorname{mode}([\alpha / \mathrm{H}])$ to be $\sim+0.3$ at $R_{\mathrm{gal}}=4 \mathrm{kpc}$, producing $\operatorname{mode}([\alpha / \mathrm{H}]) \approx 0$ at $R_{\mathrm{gal}}=7-9 \mathrm{kpc}$. This results in the following form for $\eta$ as a function of Galactocentric radius:

$\eta\left(R_{\mathrm{gal}}\right)=\frac{y_{\alpha}^{\mathrm{CC}}}{Z_{\alpha, \odot}} 10^{\left(-0.08 \mathrm{kpc}^{-1}\right)\left(R_{\mathrm{gal}}-4 \mathrm{kpc}\right)+0.3}+r-1$,

where we adopt our CCSN yield of $\mathrm{O}$ for $y_{\alpha}^{\mathrm{CC}}$ and the solar photospheric abundance of $\mathrm{O}$ of $Z_{\mathrm{O}, \odot}=0.00572$ based on Asplund et al. (2009). We plot this adopted scaling in the top panel of Fig. 3, highlighting a value of $\sim 2.15$ for the solar circle with a red dotted line. In Fig. 9 below, we show that our full model including a time-dependent SFH and radial migration produces stellar and gas phase gradients similar but not identical to those of equation (8). In Figs. 10 and 11 below, we show that the model achieves qualitative agreement with the metallicity gradient observed by APOGEE.

\subsection{Star Formation Histories}

VICE computes models in either "infall", "star formation", or "gas" mode, referring to which component of the evolutionary history the user has specified. The starburst models of Johnson \& Weinberg (2020) ran in infall mode, meaning that the gas infall rate is specified and the SFR follows from the gas surface density and adopted star formation law. Here we run VICE in star formation mode so that we achieve a specified form of the SFHs in our models. In Appendix B, we explain how we normalize the parameters of our SFHs to produce a realistic model Galaxy at the present day. In short, we take a unitless description of the time-dependence of the SFH at a given Galactocentric radius, denoted $f\left(t \mid R_{\text {gal }}\right)$, and a unitless description of the present day stellar surface density gradient, denoted $g\left(R_{\text {gal }}\right)$. We integrate $f\left(t \mid R_{\text {gal }}\right)$ with time for each annulus, assuming $R_{\text {gal }}$ to correspond to the centre of the zone, and attach a prefactor to $f\left(t \mid R_{\text {gal }}\right)$ in each annulus such that the desired gradient is achieved with a total stellar mass similar to the Milky Way. This procedure neglects the impact of radial migration, assuming that it does not significantly alter the form of $g\left(R_{\mathrm{gal}}\right)$. We demonstrate that this assumption holds in $\S 2.7$, in which we also detail our adopted form of $g\left(R_{\mathrm{gal}}\right)$. The equation derived in Appendix B can be used to calculate these prefactors for alternative models of Milky Way-like galaxies.

In the present paper, we consider four forms of the SFH, which we dub "constant", "inside-out", "late-burst", and "outer-burst". They are defined as follows: 


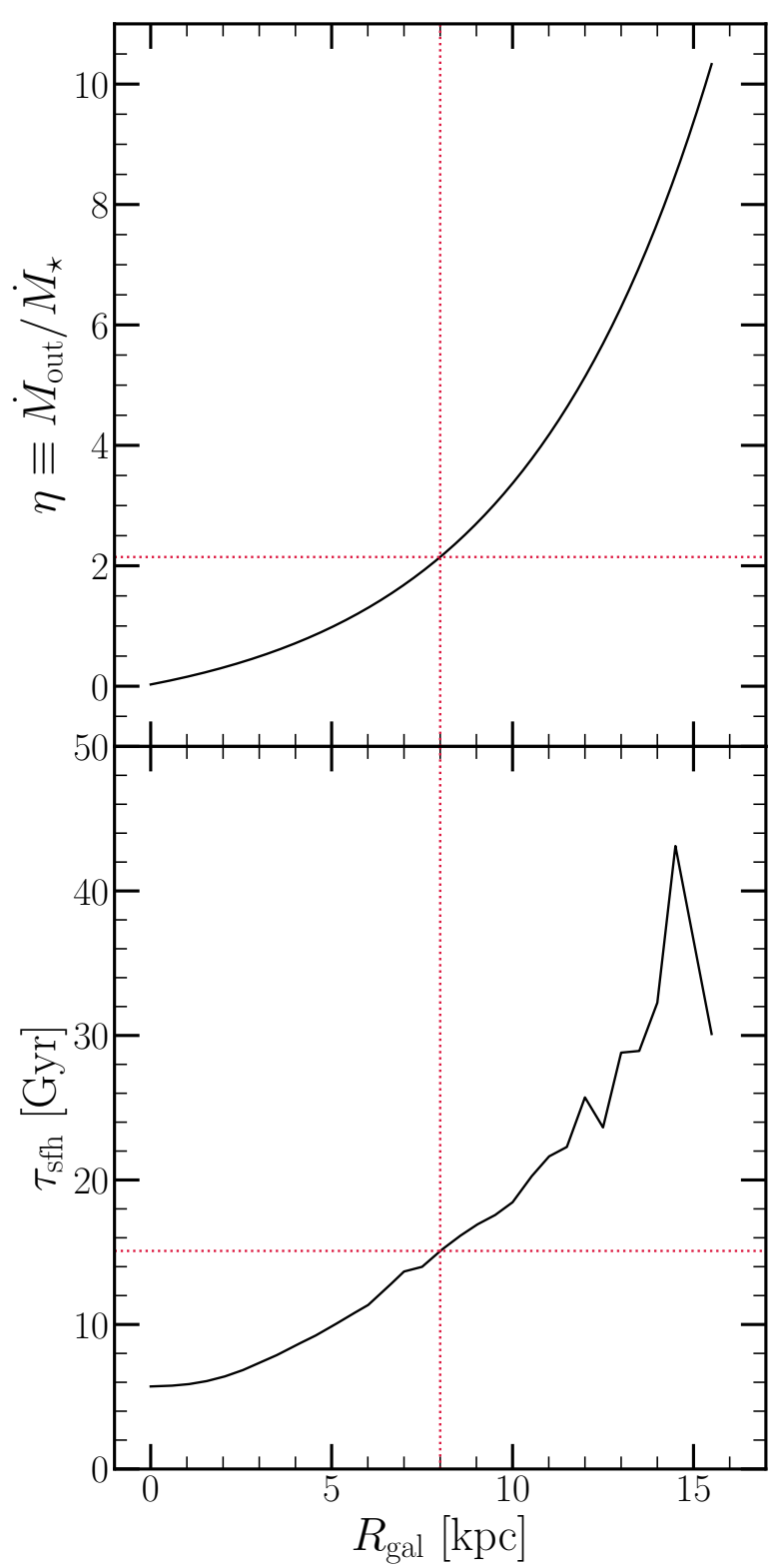

Figure 3. Top: Our implemented scaling of the mass loading factor $\eta$ with Galactocentric radius (black) as defined by equation (8). Bottom: The efolding timescales of the star formation histories of our model galaxies (black). These values come from a fit to the $\Sigma_{\star}$-age relation in bins of $R / R_{\mathrm{e}}$ for $10^{10.5}$ $10^{11} \mathrm{M}_{\odot} \mathrm{Sa} / \mathrm{Sb}$ Hubble type spiral galaxies as reported by Sánchez (2020, see discussion in $\$ 2.5$ ). The horizontal and vertical red dashed lines in both panels highlight a mass loading factor of $\eta \approx 2.15$ and a star formation timescale of $\tau_{\mathrm{sfh}} \approx 15 \mathrm{Gyr}$ at an assumed radius of the sun of $R_{\odot}=8 \mathrm{kpc}$.

- Constant: The SFH at a given radius is time-independent,

$f_{\mathrm{C}}\left(t \mid R_{\mathrm{gal}}\right)=1$.

This case is of theoretical interest because it quantifies the effect of stellar migration while removing the impact of a time-dependent SFH.

- Inside-Out: This is our fiducial SFH,

$f_{\mathrm{IO}}\left(t \mid R_{\mathrm{gal}}\right)=\left(1-e^{-t / \tau_{\text {rise }}}\right) e^{-t / \tau_{\mathrm{sfh}}}$.

We adopt this mathematical form over the somewhat more common $t e^{-t / \tau_{\text {sfh }}}$ because it allows separate control over the rising and falling phase of the SFH. Equation (10) has a maximum near $\tau_{\text {rise }}$, which in this paper we set to $2 \mathrm{Gyr}$ at all radii. This form produces a peak in star formation at lookback times of $\sim 11 \mathrm{Gyr}$, roughly corresponding to a redsfhit of $z \approx 2.5$. We discuss the choice of $\tau_{\text {sfh }}$ below.

- Late-Burst: In this model, the inside-out SFH is modified to exhibit a recent, slow "burst" in star formation described by a Gaussian in time:

$f_{\mathrm{LB}}\left(t \mid R_{\mathrm{gal}}\right)=f_{\mathrm{IO}}\left(t \mid R_{\mathrm{gal}}\right)\left(1+A_{b} e^{-\left(t-t_{b}\right)^{2} / 2 \sigma_{b}^{2}}\right)$

where $A_{b}$ is a dimensionless parameter describing the strength of the starburst, $t_{b}$ is the time of the local maximum in the SFH during the burst, and $\sigma_{b}$ is the width of the Gaussian describing it. To approximate the findings of Mor et al. (2019) and Isern (2019), we adopt $A_{b}=1.5, t_{b}=11.2 \mathrm{Gyr}$, and $\sigma_{b}=1 \mathrm{Gyr}$, finding that $A_{b}=1.5$ with a declining $f_{\mathrm{IO}}\left(t \mid R_{\mathrm{gal}}\right)$ produces a local maximum SFR that is a factor of $\sim 2$ larger than the preceding local minimum.

- Outer-Burst: A variation of the late-burst model in which only zones at $R_{\text {gal }} \geq 6 \mathrm{kpc}$ experience the starburst, with inner regions following the inside-out SFH. Because the empirical evidence for elevated recent star formation comes from local observations, it is useful to investigate the case where it is confined to the outer Galaxy. In their hydrodynamical simulation of a Milky Way-like galaxy, Vincenzo \& Kobayashi (2020) find that satellite perturbations enhance gas accretion preferentially in the outer regions.

The inside-out model is our fiducial choice, and it is the model shown in figures unless otherwise specified. The constant SFR model allows us to investigate the impact of migration when the time-dependence of the SFH is removed, and the two burst models allow us to explore the consequences of elevated recent star formation supported by some recent data. More complex scenarios with multiple bursts induced by repeated satellite passages (e.g. Lian et al. 2020a,b; Ruiz-Lara et al. 2020; Sysoliatina \& Just 2021) can be modeled easily in VICE, but we do not explore them here.

We derive the $\tau_{\text {sfh }}-R_{\text {gal }}$ relation from the data of Sánchez (2020), who presents the stellar surface density $\Sigma_{\star}$ as a function of age in bins of $R / R_{\mathrm{e}}$ for MaNGA galaxies (Bundy et al. 2015), where $R_{\mathrm{e}}$ is the half-light radius. Here we take the $M_{\star}=10^{10.5}-10^{11} \mathrm{M}_{\odot}$ bin for $\mathrm{Sa} / \mathrm{Sb}$ spirals and simultaneously fit the normalization and efolding timescale $\tau_{\text {sfh }}$ of our $f_{\mathrm{IO}}\left(t \mid R_{\text {gal }}\right)$ form to the data. Although the normalization is irrelevant to our models and determined via the method outlined in Appendix B, we adopt the resulting $\tau_{\mathrm{sfh}}-R_{\mathrm{gal}}$ relation in our models. Our adopted stellar surface density gradient (see $§ 2.7$ ) implies a present-day half-mass radius near $4 \mathrm{kpc}$. The findings of García-Benito et al. (2017) and González Delgado et al. (2014) suggest that half-light radii are marginally larger than halfmass radii. Based on equation (4) of González Delgado et al. (2014) relating the two for circular apertures, we expect our model Galaxy to have a half-light radius near $5 \mathrm{kpc}$. We therefore adopt $R_{\mathrm{e}}=5 \mathrm{kpc}$ to convert the $\tau_{\mathrm{sfh}}-R_{\mathrm{gal}} / R_{\mathrm{e}}$ relation resulting from our fit to the Sánchez (2020) data into a $\tau_{\mathrm{sfh}}-R_{\mathrm{gal}}$ relation.

We illustrate this relationship in the bottom panel of Fig. 3. The resulting timescales are long, particularly for the outer Galaxy. With a red dotted line, we highlight a value of $\tau_{\text {sfh }} \approx 15 \mathrm{Gyr}$ at an assumed orbital radius of the sun of $R_{\odot}=8 \mathrm{kpc}$. The long timescales reflect the fact that the $\Sigma_{\star}\left(\tau_{\text {lookback }}\right)$ profiles in Fig. 11 of Sánchez (2020) are fairly flat. For comparison, we have also considered the assumption that the Galactic SFH may have resembled the cosmic SFH by running models with e-folding timescales of a $\sim$ few Gyr (e.g. Madau \& Dickinson 2014). We find similar results in these cases, suggesting that our qualitative conclusions are not sensitive to the exact values of $\tau_{\text {sfh. }}$.

We plot the resulting SFHs of our models in the top row of Fig. 4 for a handful of radii. Because of the long $\tau_{\mathrm{sfh}}$, the SFR at most radii has fallen only modestly from its $2 \mathrm{Gyr}$ peak in the inside-out model. At all timesteps, the gas supply is known via the star formation efficiency timescale $\tau_{\star}$ (see $\S 2.6$ ), which is illustrated in the bottom row of Fig. 4. VICE automatically calculates the implied infall rate by comparing the amount of gas lost to outflows and star formation in a given timestep to that which is required to sustain the user-specified level of star formation at the next timestep; we assume the infalling gas to be of zero metallicity at all times. This quantity is shown in the middle row of Fig. 4. 


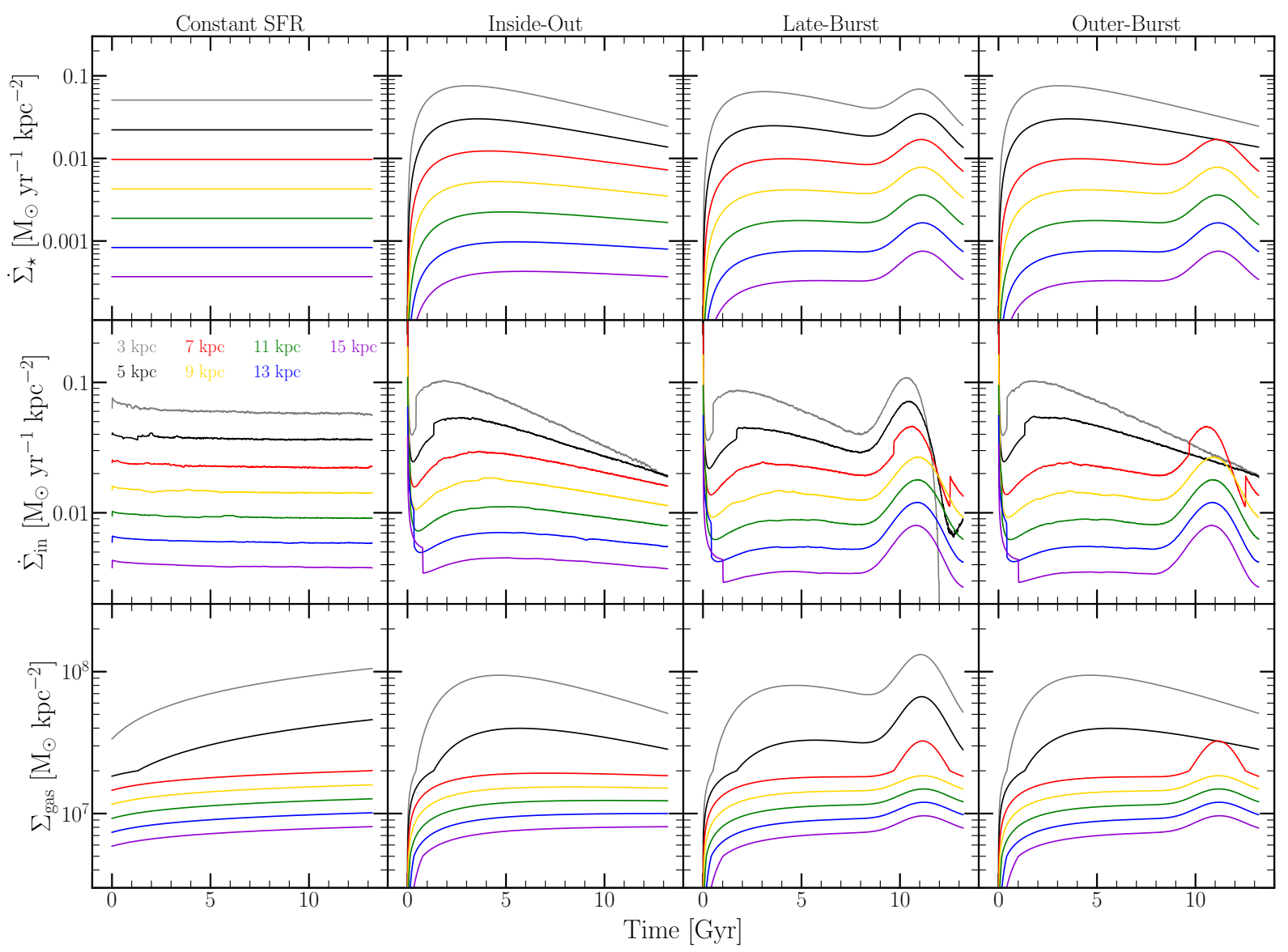

Figure 4. The surface densities of star formation $\dot{\Sigma}_{\star}$ (top row), infall $\dot{\Sigma}_{\text {in }}$ (middle row), and gas $\Sigma_{\mathrm{g}}$ (bottom row) as functions of simulation time for our four fiducial SFHs: constant (far left), inside-out (left middle), late-burst (right middle), and outer-burst (far right). We plot curves for the rings whose inner radii are $3 \mathrm{kpc}$ (grey), $5 \mathrm{kpc}$ (black), $7 \mathrm{kpc}$ (red), $9 \mathrm{kpc}$ (yellow), $11 \mathrm{kpc}$ (green), $13 \mathrm{kpc}$ (blue), and $15 \mathrm{kpc}$ (purple) (see equations (9), (10), and (11) for the mathematical definition of each SFH).

\subsection{Star Formation Efficiency}

The term "star formation efficiency" (SFE) is somewhat overloaded in the literature. In the star formation and feedback community, it usually refers to the fraction of a molecular cloud's mass that will eventually be converted into stars. In the chemical evolution literature, however, it typically refers to the inverse timescale relating the SFR within some star forming reservoir to the mass of gas in that region: $\tau_{\star} \equiv \Sigma_{\mathrm{g}} / \dot{\Sigma}_{\star}$. High (Low) values of $\tau_{\star}$ indicate slow (fast) conversion of gas and thus low (high) SFE; when we refer to SFE here, we mean the definition based on this timescale. In the star formation and feedback literature, $\tau_{\star}$ is sometimes referred to as the "depletion time", though here we follow the terminology of Weinberg et al. (2017) who call it the "star formation efficiency timescale."

Based on the findings of Kennicutt (1998), it is common practice in the chemical evolution literature to adopt a single power-law describing the relationship between the surface densities of gas and star formation $\Sigma_{\mathrm{g}}$ and $\dot{\Sigma}_{\star}$, often referred to as the star formation law or the Kennicutt-Schmidt relation:

$\dot{\Sigma}_{\star} \propto \Sigma_{\mathrm{g}}^{N}$.

Kennicutt (1998) finds $N=1.4 \pm 0.15$ relating the total $\dot{\Sigma}_{\star}$ and $\Sigma_{\mathrm{g}}$ within the disc across a sample of quiescent spiral galaxies and infrared and circumnuclear starbursts. However, recent studies have found evidence that much of the observed scatter in this relation is physical in origin (de los Reyes \& Kennicutt 2019) and that there are significant breaks in both the power-law index and zero-points (Kennicutt \& de los Reyes 2021). Some of the uncertainty surrounding the details of the star formation law is a consequence of the ongoing debate about the CO-to- $\mathrm{H}_{2}$ conversion factor (Kennicutt \& Evans 2012; Liu, Gao \& Greve 2015). Although Ellison et al. (2021) demonstrate that there are significant galaxy-to-galaxy variations in the star formation law, de los Reyes \& Kennicutt (2019) argue that the mean trend is still a reasonable recipe for Galaxy evolution models. However, for our purposes we also need the dependence of $\dot{\Sigma}_{\star}$ on $\Sigma_{g}$ within a galaxy.

Krumholz et al. (2018) compare theoretically motivated star formation laws to the observations of Bigiel et al. (2010) and Leroy et al. (2013) (see their Fig. 2). We find that the following by-eye fit to the power-law index $N$ is a reasonable description of the aggregate data:

$$
N= \begin{cases}1.0 & \left(\Sigma_{\mathrm{g}} \geq \Sigma_{\mathrm{g}, 2}\right), \\ 3.6 & \left(\Sigma_{\mathrm{g}, 1} \leq \Sigma_{\mathrm{g}} \leq \Sigma_{\mathrm{g}, 2}\right), \\ 1.7 & \left(\Sigma_{\mathrm{g}} \leq \Sigma_{\mathrm{g}, 1}\right)\end{cases}
$$

where $\Sigma_{\mathrm{g}, 1}=5 \times 10^{6} \mathrm{M}_{\odot} \mathrm{kpc}^{-2}$ and $\Sigma_{\mathrm{g}, 2}=2 \times 10^{7} \mathrm{M}_{\odot} \mathrm{kpc}^{-2}$. The apparent linearity of the relationship above $\sim 2 \times 10^{7} \mathrm{M}_{\odot} \mathrm{kpc}^{-2}$ suggests that in this regime, star formation proceeds at the highest efficiency, and that $\tau_{\star} \equiv \Sigma_{\mathrm{g}} / \dot{\Sigma}_{\star}=$ constant. The observational results of Leroy et al. (2013) and Kennicutt \& de los Reyes (2021) would suggest that these are the surface densities at which the molecular fraction $f_{\text {mol }}=M_{\mathrm{H}_{2}} /\left(M_{\mathrm{H}_{2}}+M_{\mathrm{HI}}\right) \approx 1$. We therefore adopt the assumption that above $\Sigma_{\mathrm{g}}=2 \times 10^{7} \mathrm{M}_{\odot} \mathrm{kpc}^{-2}, \tau_{\star}$ reaches its minimum value, and increases with decreasing $f_{\text {mol }}$. We denote this value as $\tau_{\text {mol }}$, the value of $\tau_{\star}$ for a gas reservoir with $f_{\text {mol }}=1$. This identification, combined with our three-component power-law index $N$ results in the following final form for our adopted star formation law:

$$
\dot{\Sigma}_{\star}= \begin{cases}\Sigma_{\mathrm{g}} \tau_{\mathrm{mol}}^{-1} & \left(\Sigma_{\mathrm{g}} \geq \Sigma_{\mathrm{g}, 2}\right), \\ \Sigma_{\mathrm{g}} \tau_{\mathrm{mol}}^{-1}\left(\frac{\Sigma_{\mathrm{g}}}{\Sigma_{\mathrm{g}, 2}}\right)^{2.6} & \left(\Sigma_{\mathrm{g}, 1} \leq \Sigma_{\mathrm{g}} \leq \Sigma_{\mathrm{g}, 2}\right), \\ \Sigma_{\mathrm{g}} \tau_{\text {mol }}^{-1}\left(\frac{\Sigma_{\mathrm{g}, 1}}{\Sigma_{\mathrm{g}, 2}}\right)^{2.6}\left(\frac{\Sigma_{\mathrm{g}}}{\Sigma_{\mathrm{g}, 1}}\right)^{0.7} & \left(\Sigma_{\mathrm{g}} \leq \Sigma_{\mathrm{g}, 1}\right) .\end{cases}
$$

We choose the power-law indices such that this formalism is consis- 


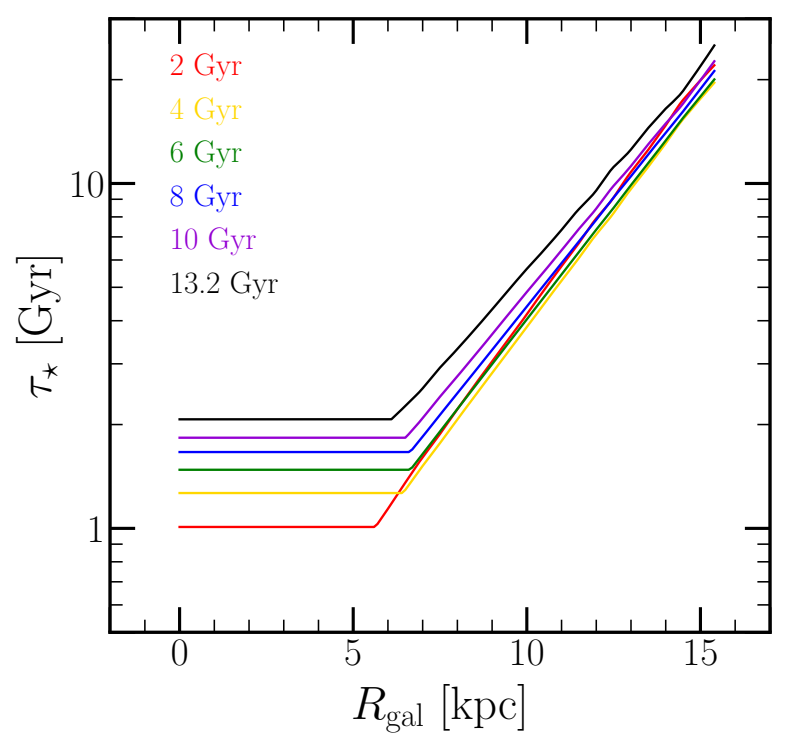

Figure 5. The star formation efficiency timescale $\tau_{\star}$ as a function of Galactocentric radius at simulation times of 2 Gyr (red), 4 Gyr (yellow), 6 Gyr (green), 8 Gyr (blue), 10 Gyr (purple), and 13.2 Gyr (the present day, black) predicted by our fiducial model.

tent with equation (13), and prefactors are added to ensure piece-wise continuity. In implementation, VICE requires the $\tau_{\star}-\dot{\Sigma}_{\star}$ relation when running in star formation mode and the $\tau_{\star}-\Sigma_{\mathrm{g}}$ relation when running in infall and gas modes. Both follow algebraically from this relationship given the substitution $\tau_{\star} \equiv \Sigma_{\mathrm{g}} / \dot{\Sigma}_{\star}$.

Based on the observed Kennicutt-Schmidt relation at different redshifts, Tacconi et al. (2018) suggest that $\tau_{\text {mol }}$ should scale with redshift $z$ and with the deviation from the star forming main sequence $\delta \mathrm{MS}$ via $\tau_{\mathrm{mol}} \propto(1+z)^{-0.6} \delta \mathrm{MS}^{-0.44}$. We do not account for the effect of $\delta \mathrm{MS}$ in our models, but we do incorporate the redshift dependence. For redshifts of $z \lesssim 3$, encompassing most of the timesteps in our models, a reasonable approximation to the $t-z$ relation assuming typical $\Lambda \mathrm{CDM}$ cosmology is given by:

$\frac{t}{t_{0}} \approx(1+z)^{-5 / 4}$,

where $t_{0}$ is the present-day age of the universe, and $t$ is not simulation time but the age of the universe. Plugging this relation into the Tacconi et al. (2018) scaling yields

$\tau_{\mathrm{mol}}=\tau_{\mathrm{mol}, 0}\left(t / t_{0}\right)^{12 / 25} \approx \tau_{\mathrm{mol}, 0}\left(t / t_{0}\right)^{1 / 2}$,

where $\tau_{\mathrm{mol}, 0}$ is simply $\tau_{\mathrm{mol}}$ at the present day. We generalize this formula to the following form:

$\tau_{\text {mol }}=\tau_{\text {mol, } 0}\left(t / t_{0}\right)^{\gamma}$

In this paper we present models which adopt $\tau_{\text {mol, } 0}=2$ Gyr (Leroy et al. 2008, 2013; Tacconi et al. 2018) and $\gamma=1 / 2$ based on this argument. We have also run simulations witht $\tau_{\mathrm{mol}, 0}=1 \mathrm{Gyr}$ and with $\gamma$ $=0$ (a time-independent $\tau_{\text {mol }}$ ), as well as combinations of the two, and found similar results. In all timesteps and annnuli, VICE infers $\Sigma_{\mathrm{g}}$ from $\dot{\Sigma}_{\star}$ given our equations (14) and (17).

In Fig. 5, we plot $\tau_{\star}$ as a function of $R_{\text {gal }}$ at six different time stamps predicted by our fiducial, inside-out SFH model. At $R_{\text {gal }} \lesssim 6$ $\mathrm{kpc}, \tau_{\star}$ is near $\tau_{\mathrm{mol}}$ at all times, implying a molecular fraction of unity at these radii. Although this prediction is likely unrealistic because $21-\mathrm{cm}$ line observations suggest the presence of neutral hydrogen as close to the Galactic centre as several hundred pc (Kalberla \& Kerp 2009), we find in practice that changing our assumptions about the star formation law does not impact our conclusions. In exploratory work for this paper, we investigated purely linear, purely power-law, and broken power-law characterizations, finding similar predictions in all cases. In general we find that the detailed form of the SFH, and to some extent the time-dependence of radial migration, exert much

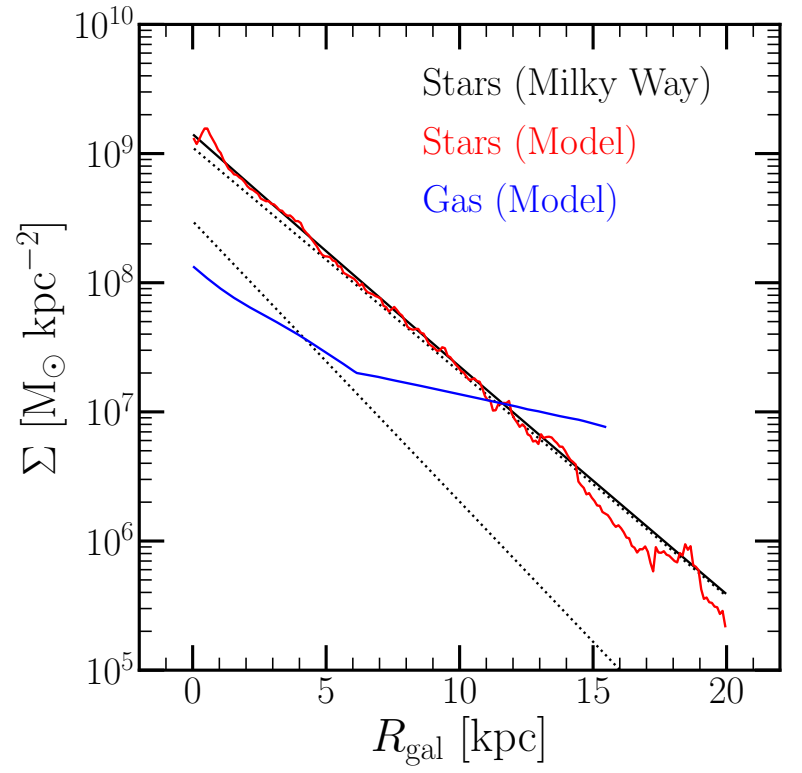

Figure 6. The surface density of gas (blue) and stars (red) as predicted by our fiducial model. The dotted black lines denote thin and thick disc profiles with scale lengths of $R_{t}=2.5 \mathrm{kpc}$ and $R_{T}=2.0 \mathrm{kpc}$, respectively, with a ratio of $\Sigma_{T} / \Sigma_{t}=0.27$ at $R_{\text {gal }}=0$ (i.e. the thin disc profile has the higher normalization). The solid black line denotes the sum of the two; this is the stellar surface density gradient of the Milky Way as reported by Bland-Hawthorn \& Gerhard (2016), renormalized according to our adopted total stellar mass of $(5.17 \pm 1.11) \times 10^{10} \mathrm{M}_{\odot}($ Licquia \& Newman 2015).

greater power in establishing the model predictions than does the star formation law. Nonetheless it is an interesting puzzle that a $\dot{\Sigma}_{\star}-\Sigma_{\mathrm{g}}$ relation informed by the observed population-averaged trends and the normalization of SFHs implied by the stellar mass of the Milky Way predicts results in tension with the observed $\mathrm{HI}$ distribution. Because the star formation law has minimal impact on our results, we do not pursue this question further here.

\subsection{Surface Density Gradient}

As discussed in $\S 2.5$, Appendix B presents the recipe by which we select a unitless function describing the stellar surface density gradient $g\left(R_{\text {gal }}\right)$. In setting the normalization, our model ensures that the integral of $g\left(R_{\mathrm{gal}}\right)$ over the surface area of the disc predicts a total stellar mass in agreement with that observed for the Milky Way. For this value, we adopt $M_{\star}^{\mathrm{MW}}=5.17 \times 10^{10} \mathrm{M}_{\odot}$ from Licquia \& New$\operatorname{man}\left(2015, \pm 1.11 \times 10^{10} \mathrm{M}_{\odot}\right)$. This is the total disc mass only; when the bulge is included, the total becomes $(6.08 \pm 1.17) \times 10^{10} \mathrm{M}_{\odot}$. Since we are modeling only the disc populations here, we omit the contribution from the bulge to the total mass budget.

We adopt a double exponential form for $g\left(R_{\mathrm{gal}}\right)$, describing the thin and thick disc components of the Galaxy:

$g\left(R_{\mathrm{gal}}\right)=e^{-R_{\mathrm{gal}} / R_{t}}+\frac{\Sigma_{T}}{\Sigma_{t}} e^{-R_{\mathrm{gal}} / R_{T}}$,

where $R_{t}$ and $R_{T}$ are the scale radii of the thin and thick discs, respectively, and $\Sigma_{T} / \Sigma_{t}$ is the ratio of their surface densities at $R_{\mathrm{gal}}=$ 0 . We adopt $R_{t}=2.5 \mathrm{kpc}, R_{T}=2.0 \mathrm{kpc}$, and $\Sigma_{T} / \Sigma_{t}=0.27$ based on the findings of Bland-Hawthorn \& Gerhard (2016). We plot the single exponential forms of each disc component as dotted black lines in Fig. 6, with the solid black line denoting the sum of the two.

We plot the resultant surface density gradients from our fiducial, inside-out SFH model in Fig. 6 as well, with red denoting the stellar gradient and the gas in blue. The stellar gradient very nearly follows our target gradient (equation 18) denoted by the solid black line. Stellar migration has not altered the overall form of the gradient, simply introducing scatter around the adopted trend. Although there are slight enhancements at small $R_{\text {gal }}$ and deficits at large $R_{\text {gal }}$, the agreement is excellent in the regions of the Galaxy where the gradient 
is best constrained observationally. The $R_{\mathrm{gal}}>15.5 \mathrm{kpc}$ populations are composed entirely of stars that migrated there, since that is the radius at which we shut off star formation. The gas gradient shows a break in the scale radius near $R_{\text {gal }} \approx 6 \mathrm{kpc}$. This is a consequence of our adopted star formation law; at $\Sigma_{\mathrm{g}}=2 \times 10^{7} \mathrm{M}_{\odot} \mathrm{kpc}^{-2}$ the relation changes from linear at higher densities to $\dot{\Sigma}_{\star} \sim \Sigma_{\mathrm{g}}^{3.6}$ at lower densities (see discussion in $§ 2.6$ ).

Although we adopt the Licquia \& Newman (2015) disc stellar mass of the Milky Way here, we find similar results when taking a value which differs even by an order of magnitude. If we used a linear star formation law, then our chemical evolution would be independent of the mass normalization as it is in corresponding one-zone models (Spitoni, Vincenzo \& Matteucci 2017; Weinberg et al. 2017; Belfiore et al. 2019). The mass normalization enters our calculation because it affects the transition between the linear and non-linear regimes of our star formation law (equation 14), but the impact on abundance evolution is minimal.

\subsection{Summary}

In summary, our fiducial model has an inside-out SFH with e-folding timescales derived from the observations of Sánchez (2020, see discussion in $\S 2.5$ ). Radial migration proceeds in a manner in which our model stellar populations have a change in radius $\Delta R_{\text {gal }}$ informed from the h277 hydrodynamical simulation (Christensen et al. 2012; Zolotov et al. 2012; Loebman et al. 2012, 2014; Brooks \& Zolotov 2014 , see discussion in $\S 2.1$ ). In the baseline model, stars move to their final radius with a $\sqrt{\text { age }}$ dependence (Frankel et al. 2018, 2020, see discussion in 2.2). Using VICE to calculate abundances for $\mathrm{O}$ and $\mathrm{Fe}$ in this paper, our supernova yields are adopted from Johnson \& Weinberg (2020), who in turn take these values from Weinberg et al. (2017) (see $\S 2.3$ ). Outflows are characterized such that the equilibrium abundance of oxygen under a constant SFH follows an abundance gradient in agreement with observational results in the Milky Way (see $\S 2.4$ ). Our star formation law is based on the Bigiel et al. (2010) and Leroy et al. (2013) data presented in comparison with theoretical models in Krumholz et al. (2018, see $§ 2.6)$. To describe the stellar surface density gradient, we adopt the two-exponential form describing the thin and thick discs from Bland-Hawthorn \& Gerhard (2016, see $\S 2.7$ ). We adopt the Kroupa (2001) IMF throughout this paper.

Our selection of star particles from h277 yields a sample of $1,751,765$ candidate analogues with disc-like kinematics at the present day (see discussion in $\S 2.1$ ). We take $\Delta R_{\text {gal }}=100 \mathrm{pc}$ as the width of each annulus from $R_{\text {gal }}=0$ to $20 \mathrm{kpc}$ and a timestep size of $\Delta T=10 \mathrm{Myr}$ from $T=0$ to $13.2 \mathrm{Gyr}$. With the resulting 200 zones and 1,321 timesteps (one extra so that age $=0$ stars are included), we let VICE form $n=8$ stellar populations per zone per timestep, resulting in 2,113,600 total stellar populations with predicted masses and abundances. We set the SFR to zero beyond $R_{\text {gal }}=15.5 \mathrm{kpc}$; stellar populations do form beyond this radius and are part of the computational overhead, but they have zero mass and thus do not contribute to the chemical evolution in our models. This results in 1,627,472 stellar populations with non-zero masses and abundances, comparable to the total number of disc particles in our sample from h277. These simulations run in $\sim 5$ hours on a single core with a $3 \mathrm{GHz}$ processor and take up $\sim 235 \mathrm{MB}$ of disc space per output, including the extra data that we record for each stellar population's analogue star particle. We have also run variations with $n=2$ stellar populations per zone per timestep, finding similar results in all cases.

We have run VICE for all four of our SFHs, all four migration models, and all four variations in $\tau_{\text {mol }}$ noted in $\S 2.6$ - a total of 64 simulations, as well as a variety of other test cases. For many of our results, only the SFH variations have a substantial impact. We discuss the impact of migration or $\tau_{\text {mol }}$ variations where they are relevant.

\section{COMPARISON TO OBSERVATIONS}

We begin the comparison of our model predictions to observational data with the distribution of stellar populations in the $[\mathrm{O} / \mathrm{Fe}]-[\mathrm{Fe} / \mathrm{H}]$ plane. We separate stars into bins based on their present-day Galactic regions defined by five bins in $R_{\text {gal }}(3-5,5-7,7-9,9-11$, and $11-13 \mathrm{kpc})$ and three bins in $|z|(0-0.5,0.5-1$, and $1-2 \mathrm{kpc})$. Within each of the resulting 15 regions, we sample 10,000 stars at random from our baseline inside-out SFH model. Since stars in VICE are stand-ins for entire stellar populations, we let the probability of sampling one of them be proportional to its present day mass. We plot the results of this procedure in Fig. 7, colour-coding each stellar population by its birth radius; for visual reference, we also plot the gas-phase track which resulted from the $R_{\text {gal }}=8 \mathrm{kpc}$ annulus with the post-processing migration model in a black solid line in all panels.

In Fig. 7, we note that high- $\alpha$ sequence stars are predicted to be the dominant population at small $R_{\mathrm{gal}}$ and high $|z|$; conversely, the low$\alpha$ population dominates the statistics at large $R_{\text {gal }}$ and low $|z|$. This is consistent with the observational results of Hayden et al. (2015), who present a density map in the $[\alpha / \mathrm{M}]-[\mathrm{M} / \mathrm{H}]$ plane for the same Galactic regions (see their Fig. 4). ${ }^{1}$ Furthermore, the locus of the low- $\alpha$ sequence shifts from super-solar $[\mathrm{Fe} / \mathrm{H}]$ to sub-solar $[\mathrm{Fe} / \mathrm{H}]$ with increasing $R_{\mathrm{gal}}$, a shift which is expected given the abundance gradient that we have built into our models (see discussion in $\S 2.4$ ). The colour-coding of the points shows that the width of the low$\alpha$ sequence arises out of stellar migration: low- $\alpha$ stars with high $[\mathrm{Fe} / \mathrm{H}]$ formed in the inner Galaxy, and those with low $[\mathrm{Fe} / \mathrm{H}]$ formed in the outer Galaxy. The low- $\alpha$ locus thus represents a superposition of populations achieved by radial migration rather than an evolutionary sequence, the interpretation proposed by, e.g., Schönrich \& Binney (2009a) and Nidever et al. (2014).

In Fig. 7 one can clearly see the imprint of evolutionary tracks from different radii, appearing in the same present-day $R_{\mathrm{gal}}$ bin because of radial mixing. Though this is to some extent a consequence of the discretization of the Galaxy disc in our model, it also arises out of correlated fluctuations in the SN Ia rate (see discussion in $\S 3.1$ ). Observational scatter would wash out the appearance of distinct tracks, and intrinsic scatter might blur them if our chemical evolution model was less idealized. Our model reproduces several of the qualitative trends found by Hayden et al. (2015), but the distribution is less obviously bimodal. We quantify this point in $\S 3.3$ below. If we remake Fig. 7 for any of our other SFHs, or for our alternative migration or star formation efficiency prescriptions, the appearance is qualitatively similar. There are significant quantitative differences in some cases, which we discuss in subsequent subsections.

\subsection{Abundance Gradients}

The left panel of Fig. 8 shows gas phase $[\mathrm{O} / \mathrm{Fe}]-[\mathrm{Fe} / \mathrm{H}]$ evolutionary tracks at several radii assuming the inside-out SFH, using either our post-processing (dotted) or diffusion (solid) migration prescriptions (see $\S 2.2$ and Fig. 2). For the post-processing model the tracks are smooth and simply follow the predictions of one-zone GCE models with the parameters appropriate to each radius - with postprocessing, radial migration has no impact on the gas-phase evolution. However, some of the tracks in the diffusion model are notably different, especially at early times and large radii. These differences arise because radial migration can transport stars significantly over the timescale of SN Ia enrichment, as discussed in §2.1.

To demonstrate this point, the right panel of Fig. 8 plots a proxy

1 In Hayden et al. (2015), [M/H] represents an overall scaling of elements with solar mixture, and $[\alpha / \mathrm{M}]$ represents a scaling of $\alpha$-elements with respect to others. To a good approximation they are proxies for $[\mathrm{Fe} / \mathrm{H}]$ and $[\mathrm{O} / \mathrm{Fe}]$, respectively, and we will treat them as such in this paper. 


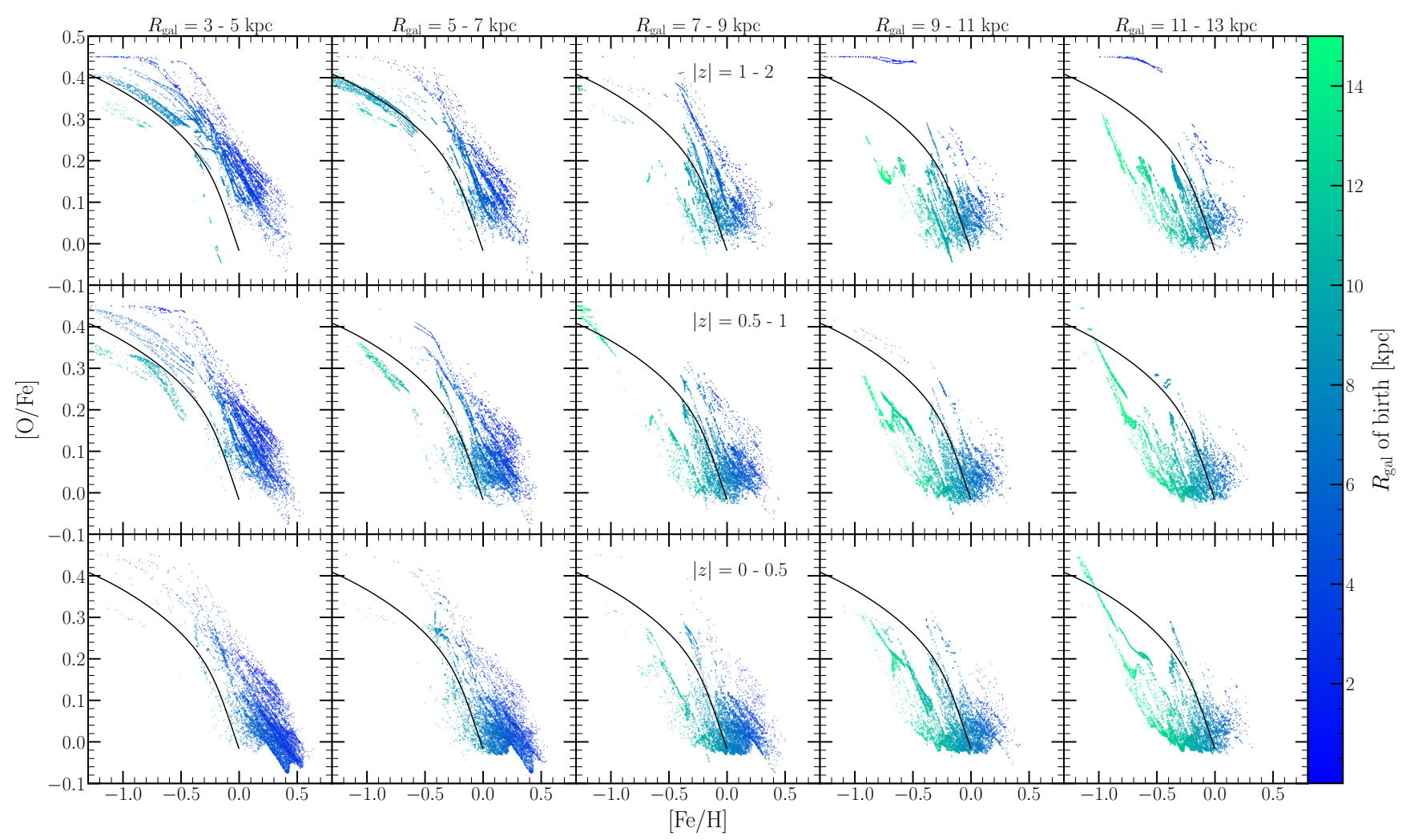

Figure 7. $[\mathrm{O} / \mathrm{Fe}]-[\mathrm{Fe} / \mathrm{H}]$ diagrams for 15 Galactic regions spanning five bins in $\boldsymbol{R}_{\text {gal }}$ and three in $|z|$. Each region has its own panel, with radial bins shown in columns denoted at the top, and with bins in $|z|$ shown in rows denoted in text in the middle column. For each region, we plot $N=10,000$ points sampled from our simulated stellar populations predicted by our fiducial model, where the probability of sampling is proportional to the present-day mass of each stellar population. In all panels, points are colour-coded according to the Galactocentric radius of birth of the stellar population. For reference, we plot in a solid black line in all panels the gas-phase [O/Fe]-[Fe/H] track predicted by the same $\mathrm{SFH}$ in the $R_{\mathrm{gal}}=8 \mathrm{kpc}$ annulus, but with the post-processing migration model; this curve is the same in all panels.

of the SN Ia rate ${ }^{2}$ as a function of time for the same $0.1 \mathrm{kpc}$ rings plotted in the left panel. At large radii the sources for the diffusion model exhibit large fluctuations relative to the smooth predictions of the underlying one-zone models, as migration boosts or depletes the predicted number of SN Ia progenitors. The deficits or excesses in SN Ia rate in turn drive upward or downward deviations in $[\mathrm{O} / \mathrm{Fe}]$ relative to the smooth model tracks in the left panel. As an extreme example, in the $15 \mathrm{kpc}$ annulus the SN Ia rate is nearly zero for the first $\sim 3$ Gyr, and its resulting $[\mathrm{O} / \mathrm{Fe}]$ track is nearly flat over this interval, even though the smooth model track has dropped from $[\mathrm{O} / \mathrm{Fe}]=0.45$ to $[\mathrm{O} / \mathrm{Fe}] \approx 0.2$. Although we illustrate the SN Ia rates for only a handful of rings, we have found that the variations between nearby rings are highly correlated in our models, suggesting that regions of the Galaxy move coherently in $[\mathrm{O} / \mathrm{Fe}]-[\mathrm{Fe} / \mathrm{H}]$ space; this lends further insight into the streak-like appearance of some sub-populations in Fig. 7.

Because we are analyzing a single hydrodynamic simulation, we cannot say whether the systematic depression of the SN Ia rate at large radii and early times is a general expectation or a consequence of the specific dynamical history of this galaxy. However, greater fluctuations at large $R_{\mathrm{gal}}$ and small $t$ are a natural consequence of the low SFR. These fluctuations can have a significant impact on $[\mathrm{O} / \mathrm{Fe}]-$ $[\mathrm{Fe} / \mathrm{H}]$ evolution even if their sign varies from galaxy to galaxy. The fact that $>10 \%$ of events are seen at $>10 \mathrm{kpc}$ from their host galaxies in the ASAS-SN bright SN catalog (Holoien et al. 2019) adds qualitative observational support to the argument that SN Ia progenitors may often form at significantly different radii than where

2 From our VICE outputs, the total time derivative of the Fe mass in a given annulus can be obtained by its change across a single timestep. By then subtracting the CCSN contribution (known exactly given our adopted yield and the SFR), approximately correcting for recycling, and adding back that which was lost to star formation and outflows, we obtain a simple estimate of Fe production by SNe Ia. their explosions are observed. We will show below that these SN Ia rate fluctuations lead to the formation of $\alpha$-enhanced intermediate age populations that do not arise in our post-processing radial migration model (see Fig. 13).

Fig. 9 plots the radial gradients of gas phase and stellar abundances, $[\mathrm{O} / \mathrm{H}],[\mathrm{Fe} / \mathrm{H}]$, and $[\mathrm{O} / \mathrm{Fe}]$, for our four $\mathrm{SFH}$ models. For constant SFR, the gas phase abundances closely track the target gradient that we used to set our $\eta\left(R_{\mathrm{gal}}\right)$ profile (see equation 8 ). For a declining SFR the equilibrium abundance is higher than that assumed for equation (8) (see Weinberg et al. 2017), and in the inside-out and outer-burst models the gas phase abundances rise above the target gradient at small $R_{\text {gal }}$ where the decline is fastest. In the late-burst model the inner Galaxy abundances are further boosted by enhanced late-time star formation, and a similar effect is seen at $R_{\text {gal }}=6 \mathrm{kpc}$ in the outer-burst model. In accretion-induced starbursts, re-enrichment can briefly produce super-equilibrium abundances in the gas-phase, which then decay back to the equilibrium abundance as the SFR declines (Johnson \& Weinberg 2020).

The stellar metallicity gradients are shallower than in the gas phase, and they are similar in the four models. The 16th-84th percentile range at each radius is large, typically $0.3-0.5 \mathrm{dex}$, and typically larger in $[\mathrm{Fe} / \mathrm{H}]$ than $[\mathrm{O} / \mathrm{H}]$. The mode of the stellar metallicity distribution is a noisy quantity in our 0.1-kpc rings, so points in Fig. 9 show the median of the distributions. The trends for the mode are slightly steeper, as expected given the change in shape of the metallicity distribution functions (see \$3.2), and closer to the target gradient shown by the black solid line. We do not include observational data in this figure, but the target gradient itself is observationally motivated, so Fig. 9 implies that our models, by design, give a reasonable match to Milky Way abundance gradients.

Median $[\mathrm{O} / \mathrm{Fe}]$ values are close to solar at nearly all radii, rising at the smallest $R_{\mathrm{gal}}$ and in some models at the largest $R_{\mathrm{gal}}$. However, the spread in $[\mathrm{O} / \mathrm{Fe}]$ is large at all radii, and typical values depend 

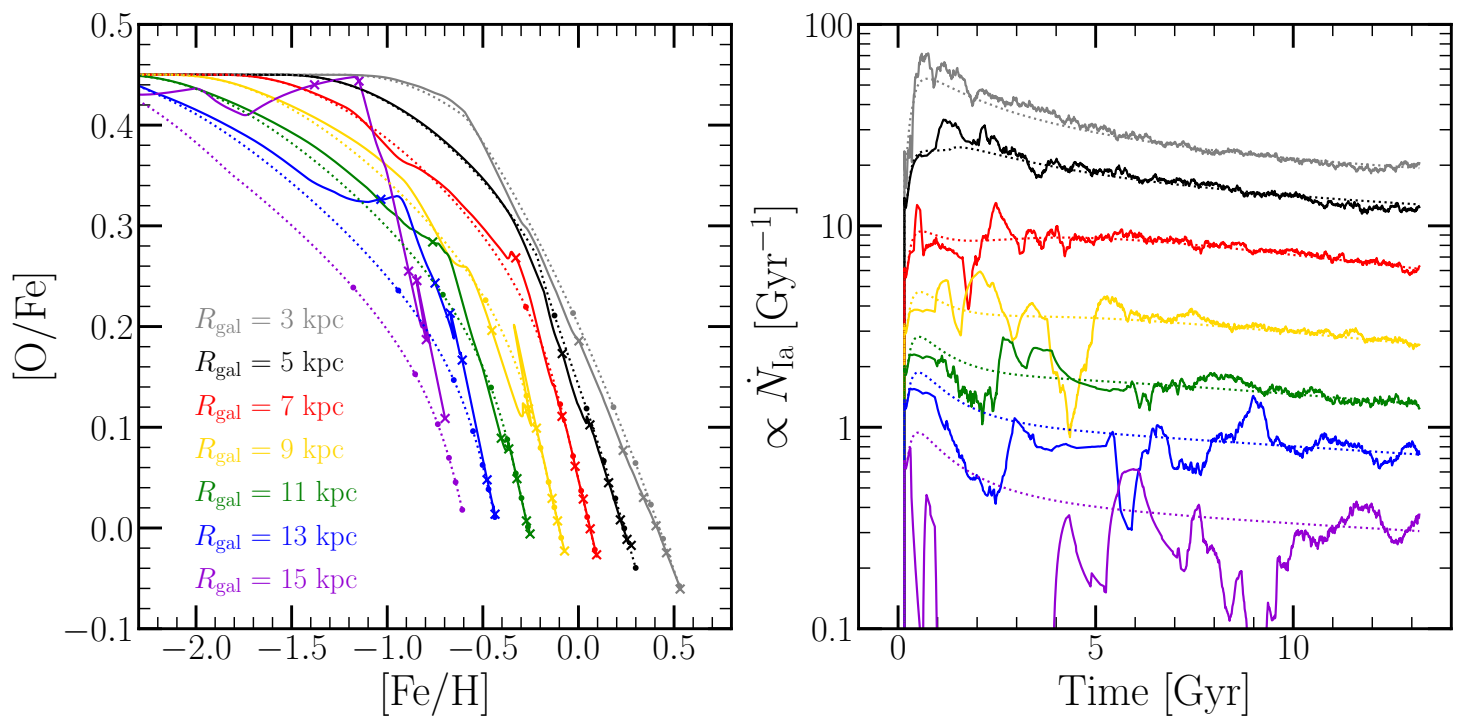

Figure 8. Left: Gas phase evolutionary tracks in the $[\mathrm{O} / \mathrm{Fe}]-[\mathrm{Fe} / \mathrm{H}]$ plane for our inside-out SFH with either post-processing (dotted lines) or diffusion (solid lines) migration models. We plot tracks for seven of our $\Delta R_{\mathrm{gal}}=100 \mathrm{pc}$ rings, colour-coded according to their Galactocentric radius and denoted by the legend in the lower-left. We mark simulation times of 2, 4, 6, 8, 10, and $13.2 \mathrm{Gyr}$ in X's for the diffusion model and points for the post-processing model. Right: As a function of simulation time, a proxy for the SN Ia rate using the total time-derivative of the Fe mass in a given annulus, calculated by subtracting the contribution from recycling and CCSN enrichment and adding back that lost to star formation and outflows. We show these rates for the same rings as in the left-hand panel, multiplying them by various prefactors to improve clarity.

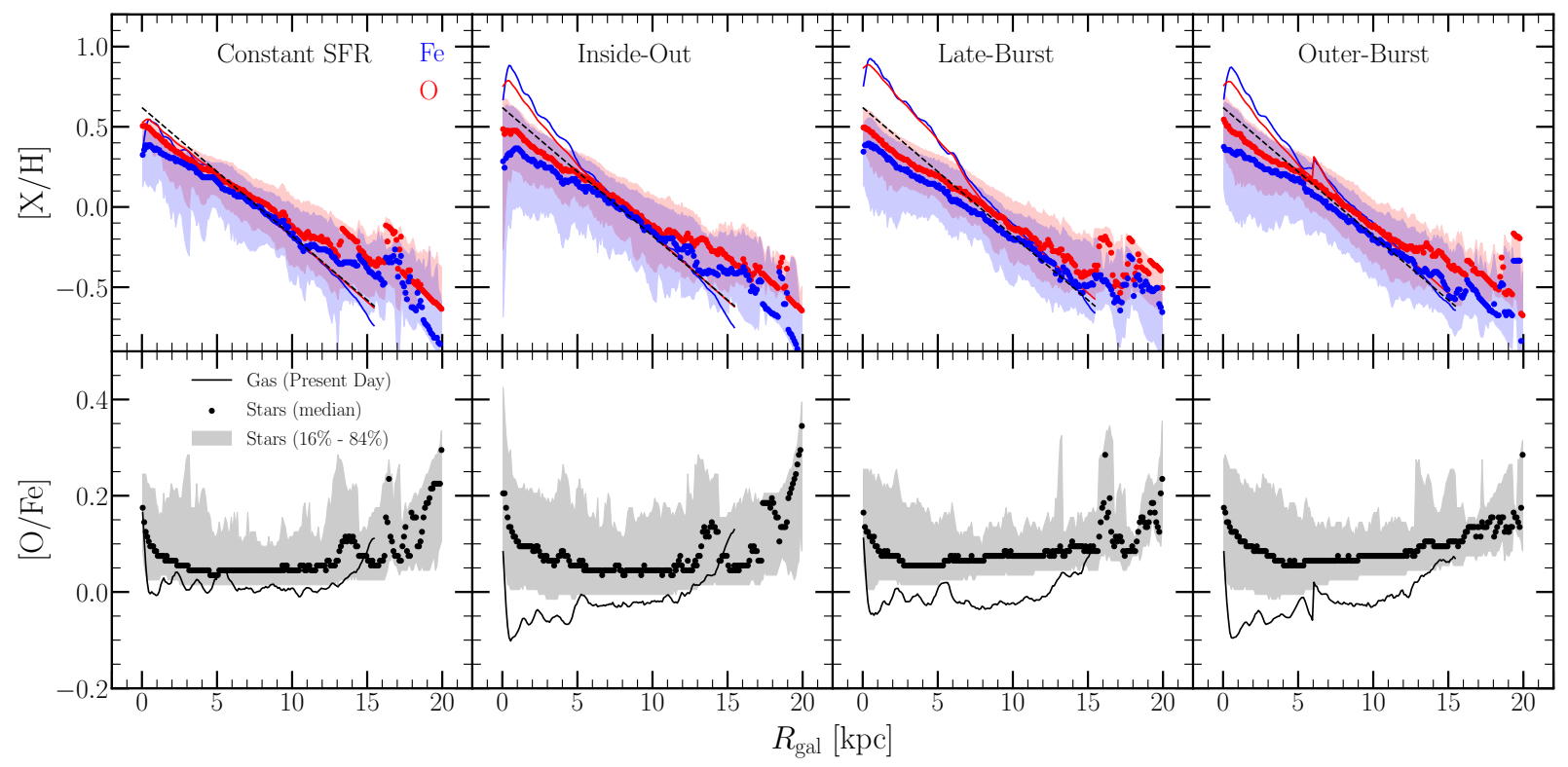

Figure 9. Radial abundance gradients in $[\mathrm{O} / \mathrm{H}]$ (top, red), [Fe/H] (top, blue), and [O/Fe] (bottom) for our four SFHs with diffusion migration - constant (far left), inside-out (left-middle), late-burst (right-middle), and outer-burst (far right). We plot the gas-phase abundance at the present day as a function of Galactocentric radius in solid lines. Points denote the median of the stellar MDF of the 100-pc width ring at a given radius, with shaded regions marking the 16th and 84th percentiles thereof. Black lines in the top panels denote our target $[\alpha / \mathrm{H}]$ gradient of mode $([\alpha / \mathrm{H}])=+0.3$ at $R_{\mathrm{gal}}=4 \mathrm{kpc}$ with a slope of $-0.08 \mathrm{kpc}{ }^{-1}$.

on $|z|$ and $[\mathrm{Fe} / \mathrm{H}]$ as shown in Fig. 7. We present a comparison to observations in $\S 3.3$ below. The larger values of $[\mathrm{O} / \mathrm{Fe}]$ beyond $R_{\text {gal }}=$ $15.5 \mathrm{kpc}$ are expected, since this is the radius at which we shut off star formation. In our models, all stellar populations at these radii migrated there, so they tend to be old and therefore $\alpha$-enhanced. The idea that outer disc populations are dominated by migration was proposed by Roškar et al. (2008b) based on simulation predictions. Radburn-Smith et al. (2012) present observational evidence for this prediction in the observations of the NGC 7793 disc. Although we are not modeling the bulge in this paper, our model predicts the disc stars in these regions to have higher $[\mathrm{O} / \mathrm{Fe}]$ than at larger $R_{\text {gal }}$, in qualitative agreement with observations (see discussion in, e.g., Duong et al. 2019; Griffith et al. 2021).

\subsection{Metallicity Distribution Functions}

Metallicity distribution functions (MDFs) and their variations with Galactic region are a core prediction of GCE models. We have computed distributions of $[\mathrm{Fe} / \mathrm{H}]$ and $[\mathrm{O} / \mathrm{H}]$ using abundances from the 16th data release (DR16; Ahumada et al. 2020; Jönsson et al. 2020) of APOGEE (Majewski et al. 2017). Abundances are determined by the APOGEE Stellar Parameters and Chemical Abundances Pipeline (ASPCAP; Holtzman et al. 2015; García Pérez et al. 2016). We restrict our sample to stars with effective temperatures of $4000 \mathrm{~K}$ $\leq T_{\text {eff }} \leq 4600 \mathrm{~K}$, surface gravities of $1.0 \leq \log g \leq 2.5$, and signalto-noise ratios of at least 100 . These cuts ensure that our sample consists of stars on the upper red giant branch, luminous enough to cover all regions of the disc, while excluding red clump stars to avoid possible abundance offsets between these stars and stars on the giant 
branch. Our observational results are similar to those shown by Hayden et al. (2015), but we use a larger data set, more recent APOGEE observations, and $[\mathrm{O} / \mathrm{H}]$ and $[\mathrm{Fe} / \mathrm{H}]$ rather than $[\alpha / \mathrm{H}]$ and $[\mathrm{M} / \mathrm{H}]$.

The left and right panels of Fig. 10 show $[\mathrm{Fe} / \mathrm{H}]$ distributions from our fiducial inside-out model and the APOGEE data, respectively, in bins of $R_{\text {gal }}$ and $|z|$. The observed midplane distributions $(|z| \leq 0.5$ kpc) show the striking phenomenon first noted by Hayden et al. (2015): a shift from a skew-negative form in the inner Galaxy to a skew-positive form in the outer Galaxy, with a roughly symmetric $[\mathrm{Fe} / \mathrm{H}]$ distribution at the solar circle. The simulation reproduces this behaviour, confirming that realistic radial migration can explain the radial dependence of the MDF shape as conjectured by Hayden et al. (2015) and illustrated in a more idealized simulation by Loebman et al. (2016).

In detail, the MDFs are not as smooth at large $R_{\text {gal }}$ as in the observations; they are not as skewed in the inner and outer disc either. Dots in the lower left panel mark the target metallicities implied by our $\eta\left(R_{\text {gal }}\right)$ prescription in equation (8). The modes of the stellar MDFs track these targets quite closely, except at $R_{\mathrm{gal}}=13-15 \mathrm{kpc}$, where the migrated population is so large compared to the in-situ population that it reshapes the peak of the MDF as well as the tails. The model also predicts a continuing increase of the mode metallicity down to $R_{\text {gal }}=$ $3-5 \mathrm{kpc}$, while the observed mode is the same at $3-5 \mathrm{kpc}$ and $5-7 \mathrm{kpc}$. We do not view this as a serious discrepancy, as it could be reduced by a moderate adjustment of the $\eta\left(R_{\text {gal }}\right)$ recipe so that the metallicity gradient in these regions is flat. We have computed such a comparison case and find that it does indeed reproduce this observational result, though it still underpredicts the skewness at small $R_{\text {gal }}$. Alternatively, the flattening of the observed gradient could be a consequence of more aggressive quenching of star formation than assumed in our models. The surface density of star formation $\dot{\Sigma}_{\star}$ in the Milky Way is known to reach a maximum at $R_{\mathrm{gal}} \approx 4 \mathrm{kpc}$ and decline by a factor of a few at smaller radii (see Fig. 1 of Peek 2009 and Fig. 2 of Fraternali \& Tomassetti 2012 and data therein). Early quenching could cutoff the MDF at high $[\mathrm{O} / \mathrm{H}]$ and $[\mathrm{Fe} / \mathrm{H}]$ if it happens before the ISM reaches equilibrium abundance. Visual inspection of Fig. 8 suggests that this process should occur around $T \approx 6-8 \mathrm{Gyr}$ if the MDF is to peak at $[\mathrm{Fe} / \mathrm{H}] \approx+0.2-0.3$.

Going up from the midplane, the observed MDFs for the four inner annuli $\left(R_{\mathrm{gal}}<11 \mathrm{kpc}\right)$ shift to lower average metallicity, and they converge in location and in shape, being roughly symmetric at $|z|=0.5$ - $1 \mathrm{kpc}$ and mildly skew-positive at $|z|=1-2 \mathrm{kpc}$. Qualitatively, the simulation reproduces this shift of mean metallicity, change of shape, and convergence of distributions at different $R_{\mathrm{gal}}$, although the model does not account for the entirety of the effect. We consider this a significant success of this simulation-based approach because our GCE model is constructed and tuned in $R_{\mathrm{gal}}$ alone, so trends with $|z|$ follow from the combinations of age-metallicity trends, age-velocity trends arising from "upside-down" disc formation and dynamical heating, and correlations between radial migration and vertical energy. Freudenburg et al. (2017) showed that chemical evolution in a vertically settling gas disc could explain the MDF trend with $|z|$ seen by APOGEE in the inner disc, and here we see similar behaviour in a more fully ab initio model. Bird et al. (2020) showed that the dynamics of the h277 simulation leads to good agreement with the observed age-velocity relation, and here we show that this success extends qualitatively to the vertical trends of chemical abundances.

Quantitatively, there are significant differences between the predicted and observed distributions above the midplane. The model MDFs from $|z|=0.5-1 \mathrm{kpc}$ are narrower and more skewed than the observed MDFs, with higher median $[\mathrm{Fe} / \mathrm{H}]$. At $|z|=1-2 \mathrm{kpc}$, the predicted MDFs are not as strikingly converged as the observed MDFs. In both the data and the model, the high- $|z|$ MDFs for the 11 - 13 and 13 - $15 \mathrm{kpc}$ annuli remain closer to their midplane counterparts. The model $[\mathrm{O} / \mathrm{Fe}]-[\mathrm{Fe} / \mathrm{H}]$ distributions in the outer Galaxy appear to show the imprint of a few large migration episodes (see Fig. 7). The smoothness of the observed MDFs at these radii and their similarity across $|z|$ suggests a more vigorous stirring.

Fig. 11 plots distributions of $[\mathrm{O} / \mathrm{H}]$ instead of $[\mathrm{Fe} / \mathrm{H}]$. The appear-

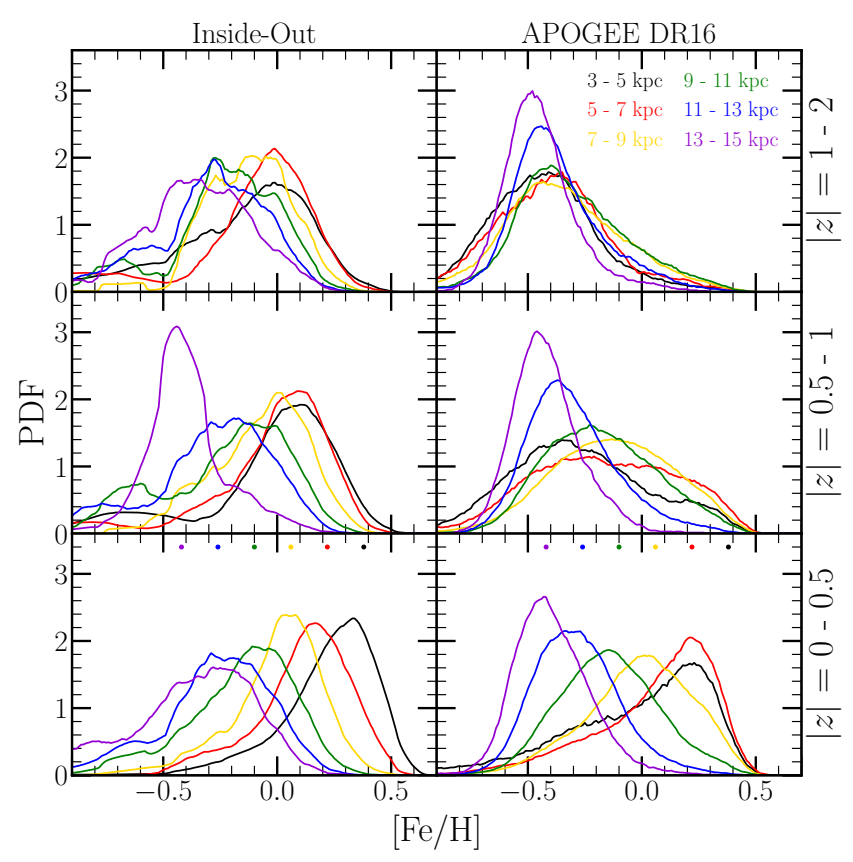

Figure 10. Metallicity distribution functions (MDFs) in $[\mathrm{Fe} / \mathrm{H}]$ predicted by our fiducial, inside-out model (left) and as observed in APOGEE DR16 (right), for stars and simulated stellar populations with present-day $|z|=0-0.5 \mathrm{kpc}$ (bottom), $0.5-1 \mathrm{kpc}$ (middle), and $1-2 \mathrm{kpc}$ (top). MDFs are shown in bins of Galactocentric radius: 3 - $5 \mathrm{kpc}$ (black), 5 - $7 \mathrm{kpc}$ (red), 7 - $9 \mathrm{kpc}$ (yellow), 9 - $11 \mathrm{kpc}$ (green), 11 - $13 \mathrm{kpc}$ (blue), and 13 - $15 \mathrm{kpc}$ (purple). The points near the top of the bottom panels denote what the mode abundance would be if it followed out target gradient of $[\mathrm{Fe} / \mathrm{H}]=+0.3$ at $R_{\text {gal }}=4 \mathrm{kpc}$ with a slope of $-0.08 \mathrm{kpc}^{-1}$ exactly, assuming the inner radius of each bin (i.e. there is no point plotted for $15 \mathrm{kpc}$ ). All distributions are smoothed with a box-car width of $[\mathrm{Fe} / \mathrm{H}] \pm 0.1$ for clarity.

ance is quite similar to Fig. 10, which is unsurprising but non-trivial given the different timescales of CCSN and SN Ia enrichment. The agreement and disagreement between the model and data are similar, though the discrepancy for $|z|=0.5-1 \mathrm{kpc}$ is somewhat clearer in $[\mathrm{O} / \mathrm{H}]$. The model's outer Galaxy MDFs are less irregular in $[\mathrm{O} / \mathrm{H}]$ than in $[\mathrm{Fe} / \mathrm{H}]$, an indication that some of the structure in the $[\mathrm{Fe} / \mathrm{H}]$ distributions is caused by the large fluctuations in the SN Ia rate as seen in Fig. 8. We have confirmed this conjecture by computing $[\mathrm{Fe} / \mathrm{H}] \mathrm{MDF}$ for the post-processing radial migration prescription, finding that they are indeed more smooth at $R_{\mathrm{gal}}=11 \mathrm{kpc}$.

The MDF predictions for our other SFH scenarios - constant SFR, late-burst, and outer-burst - are different in detail, but they show the same qualitative trends as those in Figs. 10 and 11. Our findings on the radial and vertical trends of the MDF are also similar to those of Loebman et al. (2016), who use a galaxy simulation evolved from rotating gas in a dark matter halo rather than cosmological initial conditions. The qualitative similarity of our results across different models implies that the radial and vertical trends are a generic effect of radial migration, upside-down disc formation, and dynamical heating in a galaxy with realistic abundance gradients and time evolution.

\section{$3.3[\mathrm{O} / \mathrm{Fe}]$ distributions in Bins of $[\mathrm{Fe} / \mathrm{H}]$}

Fig. 12 compares our predicted $[\mathrm{O} / \mathrm{Fe}]$ distributions in Galactic zones to those recently published by Vincenzo et al. (2021a). ${ }^{3}$ As in our $\S 3.2$, they use APOGEE DR16 with similar cuts on effective temperature, surface gravity, and signal-to-noise (see their $\S 2$ ). They correct for observational scatter as well as age-dependent and (more importantly) $|z|$-dependent selection in APOGEE to infer the

3 Vincenzo et al. (2021a) present $[\mathrm{Mg} / \mathrm{Fe}]$ distributions in their figures, but they have quantified the $[\mathrm{O} / \mathrm{Fe}]$ as well, and we use the latter here. 


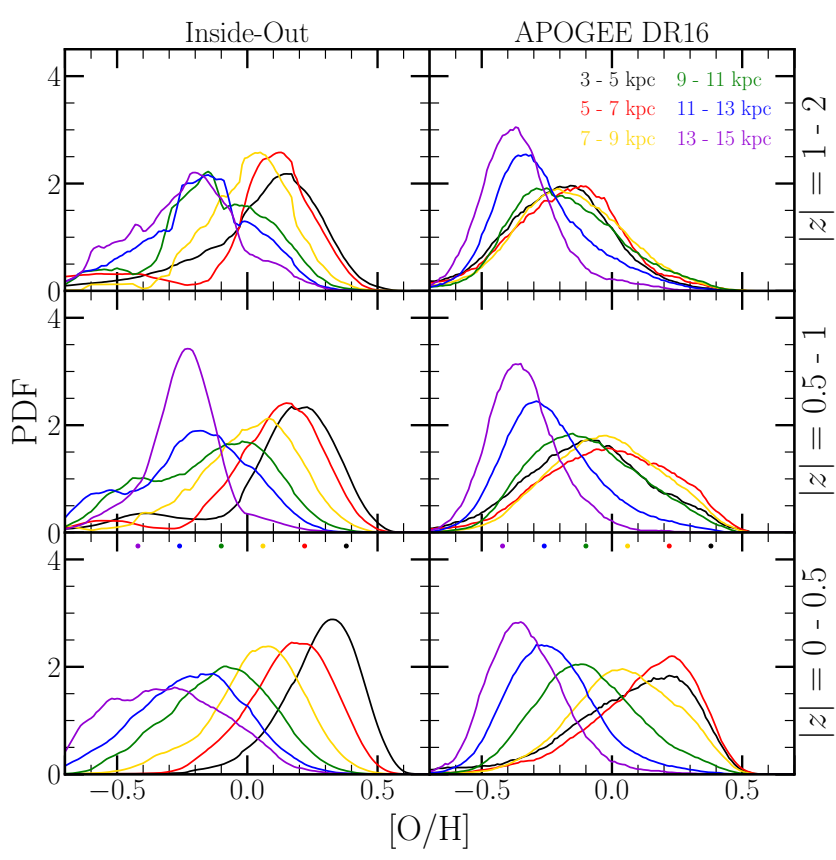

Figure 11. The same as Fig. 10, but for $[\mathrm{O} / \mathrm{H}]$.

intrinsic distribution of $[\mathrm{O} / \mathrm{Fe}]$ in bins of $[\mathrm{Fe} / \mathrm{H}]$ that would be found for an unbiased sample of long-lived disc stars. At a given $R_{\text {gal }}$ and $[\mathrm{Fe} / \mathrm{H}]$, Vincenzo et al. (2021a) fit a model comprised of two Gaussians in $[\mathrm{O} / \mathrm{Fe}]$, one each for the high- $\alpha$ and low- $\alpha$ populations. The $|z|$-dependence of the distribution follows from the empirical scale heights of these two populations, taken from Bovy et al. (2016). Dotted curves in Fig. 12 show these models integrated over the corresponding ranges in $|z|$.

Solid histograms in Fig. 12 show distributions in 0.4-dex bins of $[\mathrm{O} / \mathrm{Fe}]$ for our fiducial inside-out model. For clarity we have chosen to focus our comparison on two bins of $[\mathrm{Fe} / \mathrm{H}]$, one just above solar metallicity $(0.0 \leq[\mathrm{Fe} / \mathrm{H}] \leq 0.2)$ and one at sub-solar metallicity $(-0.4 \leq[\mathrm{Fe} / \mathrm{H}] \leq-0.2)$ where the bimodality of $[\mathrm{O} / \mathrm{Fe}]$ is most pronounced. Results for our other models are qualitatively similar, and these two $[\mathrm{Fe} / \mathrm{H}]$ bins illustrate the range of model successes and failure.

Beginning with the $[\mathrm{Fe} / \mathrm{H}]=0-0.2$ bin, we see that the model roughly reproduces the observed width of the $[\mathrm{O} / \mathrm{Fe}]$ distribution. In the midplane $(|z| \leq 0.5 \mathrm{kpc})$ zones it predicts the correct skew-positive shape, though the peak of the observed distribution is sharper than the model prediction. However, the model histograms shift towards higher $[\mathrm{O} / \mathrm{Fe}]$ with increasing $|z|$ while the peak of the observationally inferred distributions stays fixed, a discrepancy that is most obvious in the $R_{\text {gal }}=3-5 \mathrm{kpc}$ and $5-7 \mathrm{kpc}$ bins. The constancy of the observed peak is partly a consequence of the Vincenzo et al. (2021a) fitting procedure, which assumes that the location of the high- $\alpha$ and low- $\alpha$ Gaussians stay constant at a given $R_{\text {gal }}$ and $[\mathrm{Fe} / \mathrm{H}]$ and only their relative amplitudes change with $|z|$. We have gone back to the raw data histograms fit by Vincenzo et al. (2021a), and while they do allow some increase in model $[\mathrm{O} / \mathrm{Fe}]$ with $|z|$, they do not allow a shift as large as that predicted by our model. At $|z|=1-2$ and $R_{\text {gal }}=$ $3-5$ and $5-7 \mathrm{kpc}$, the number of stars contributing to the fit is 31 and 17 respectively, so while the shape of the distribution is not well constrained, the centroid is robustly determined.

This discrepancy could reflect differences in the dynamical heating history of the h277 simulation and the Milky Way. With its most recent major merger occurring at $z \approx 3$, this galaxy was previously selected for investigation for its quiescent merger history (e.g. Zolotov et al. 2012). N-body models for the tidal disruption of the Sagittarius dwarf galaxy suggest repeated pericentric passages at 1 - 2 Gyr intervals (Law \& Majewski 2010), which could trigger episodes of infall and star formation (e.g. Ruiz-Lara et al. 2020). These pericentric passages might also heat low- $\alpha$ disc populations to higher $|z|$, an effect absent in h277. Alternatively, we have investigated the impact of changing our $\eta\left(R_{\mathrm{gal}}\right)$ prescription to become constant within $R_{\mathrm{gal}}=$ $5 \mathrm{kpc}$, as discussed previously in $\S 3.2$ in the context of the $[\mathrm{Fe} / \mathrm{H}]$ gradient. This change also dampens the predicted trend of $[\mathrm{O} / \mathrm{Fe}]$ with $|z|$ by bringing the inner Galaxy's $[\mathrm{O} / \mathrm{Fe}]-[\mathrm{Fe} / \mathrm{H}]$ tracks closer together (see Figs. 7 and 8). We find that this change can account for some but not all of the discrepancy, shifting the peak of the distribution down by $\sim 0.05$ dex in the upper left panel of Fig. 12 .

Turning to the $-0.4 \leq[\mathrm{Fe} / \mathrm{H}] \leq-0.2$ bin, the model shows partial but by no means complete success. It does reproduce the breadth of the $[\mathrm{O} / \mathrm{Fe}]$ distribution at this intermediate metallicity, and in nearly all $R_{\mathrm{gal}}-|z|$ zones it predicts skewness of the correct sign. At $R_{\mathrm{gal}}=3$ - $5 \mathrm{kpc}$, the model predicts mode $([\mathrm{O} / \mathrm{Fe}]) \approx 0.3$, while the observed mode is at $[\mathrm{O} / \mathrm{Fe}] \approx 0.22$. This discrepancy is affected by our choice of $\mathrm{CCSN}$ yields, which produces an $[\mathrm{O} / \mathrm{Fe}]$ plateau at +0.45 . While this value is consistent with some observational data (e.g. the Ramírez, Allende Prieto \& Lambert 2013 data set modeled by Andrews et al. 2017), APOGEE measurements place the plateau at $[\mathrm{O} / \mathrm{Fe}] \approx+0.3$, so it is not surprising that our model overpredicts $[\mathrm{O} / \mathrm{Fe}]$ at low metallicity. Unfortunately, uncertainties in the observed abundance scales and the theoretical CCSN elemental yields remain an obstacle to sharp GCE model tests.

The most significant discrepancy with data is for $R_{\mathrm{gal}}=7-9 \mathrm{kpc}$, where the observations show a clearly bimodal $[\mathrm{O} / \mathrm{Fe}]$ distribution in all three $|z|$ ranges but the model predicts bimodality only near the midplane. This bimodality is also evident in the raw data histograms prior to model fitting and correction for selection effects (see Figs. 10 and 11 of Vincenzo et al. 2021a). The idea that radial migration could give rise to an $[\alpha / \mathrm{Fe}]$ dichotomy was proposed by Schönrich \& Binney (2009a), who noted that the evolutionary tracks at any given $R_{\text {gal }}$ would produce most stars at low $[\mathrm{O} / \mathrm{Fe}]$ and that radial mixing of these populations would produce a low- $\alpha$ "sequence" that is a superposition of these evolutionary endpoints. Nidever et al. (2014) explored this superposition scenario in the context of APOGEE observations, and Sharma et al. (2020) have recently implemented a detailed parameterized scenario matched to APOGEE. Although the superposition effect clearly operates in our model, as shown in Fig. 7, the model produces too many stars at intermediate $[\mathrm{O} / \mathrm{Fe}]$, so there is no clear minimum between the high- $\alpha$ and low- $\alpha$ peaks. As argued by Vincenzo et al. (2021a), the generic problem is that one-zone models with smooth evolutionary histories always produce most stars near the low $[\alpha / \mathrm{Fe}]$ endpoints and a much smaller peak at the high- $\alpha$ plateau, so one cannot superpose such models in a way that produces strong bimodality. The Sharma et al. (2020) model seems like a possible counter-example, but they adopt parameterized evolutionary tracks and do not demonstrate that these can be drawn from a self-consistent chemical evolution model.

The simplest way to produce stronger $[\alpha / \mathrm{Fe}]$ bimodality in our models would be to adopt a two-phase star formation model like that envisioned in two-infall scenarios of chemical evolution (e.g. Chiappini et al. 1997, 2001; Romano et al. 2010; Grisoni et al. 2017; Noguchi 2018; Palla et al. 2020; Spitoni et al. 2016, 2018, 2019, 2020, 2021). These scenarios turn down the SFR while the ISM abundances pass through the intermediate $[\alpha / \mathrm{Fe}]$ regime. Other scenarios such as large early starbursts (Clarke et al. 2019) or reverse [Fe/H] evolution with late-time outflows (Weinberg et al. 2017) could also enhance bimodality, but the late starbursts considered here are insufficient on their own. We will explore these alternative scenarios for the origin of bimodality in future work.

\subsection{The Age- $[\alpha / \mathrm{Fe}]$ Relation}

In this section, we assess the predicted age- $[\mathrm{O} / \mathrm{Fe}]$ relations of our models, using the results of Feuillet et al. (2019) as the observational benchmark. Their stellar age measurements are based on isochrone matching, using APOGEE DR14 stars for which parallax measurements are available from Gaia (Abolfathi et al. 2018; Gaia Collaboration et al. 2018). With their spatial and quality cuts, the final 


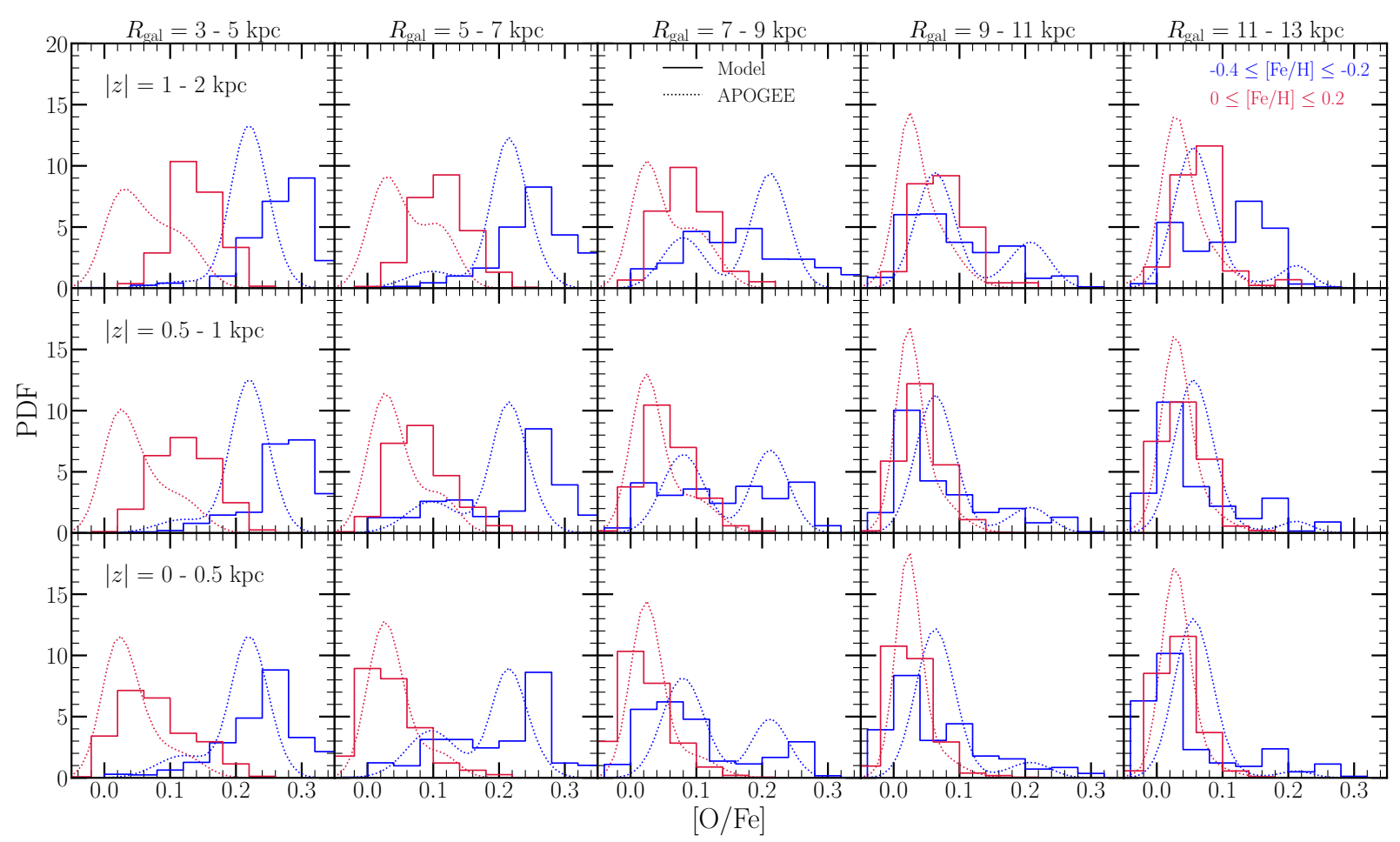

Figure 12. Predicted distributions in $[\mathrm{O} / \mathrm{Fe}]$ in 15 Galactic regions and in two bins in $[\mathrm{Fe} / \mathrm{H}]$. Columns correspond to bins in $R_{\text {gal }}$, denoted at the top of each column. Rows correspond to bins in $|z|$, denoted in text in the left-hand column. Distributions are color-coded according to the [Fe/H] the sample is drawn from, denoted by the legend in the upper right panel. Solid lines represent that predicted by our inside-out SFH in $\Delta[\mathrm{O} / \mathrm{Fe}]=0.04$ bins, while dashed lines correspond to the fits to the APOGEE DR16 data presented in Vincenzo et al. (2021a), which quantify the intrinsic distributions accounting for observational uncertainties and the APOGEE selection function.

sample consisted of 77,562 stars. In bins of [O/Fe], they assume a Gaussian distribution of log age, fitting the mean (or equivalently, the median) and standard deviation to the stars in that bin. The choice of a Gaussian distribution is driven by simplicity, enabling estimates of mean and spread for data with significant age errors, but because our model predicts non-Gaussian log age distributions at fixed $[\mathrm{O} / \mathrm{Fe}]$, the comparison to data is not free of nuance. We base most of our model comparisons below on the mass-weighted median age, simply denoting the 50th percentile of the mass-weighted age distribution of some subsample.

In the left-hand set of panels of Fig. 13, we compare the age-[O/Fe] relation in the solar neighbourhood $\left(R_{\mathrm{gal}}=7-9 \mathrm{kpc}\right.$ and $|z| \leq 0.5$ $\mathrm{kpc})^{4}$ predicted by our four migration models to the Feuillet et al. (2019) measurements, shown in red triangles. We mark the massweighted median age in bins of $[\mathrm{O} / \mathrm{Fe}]$ in each model with black squares, and plot for reference in the background each individual stellar population in the solar neighbourhood, colour-coded according to its Galactocentric radius of birth.

The median age- $[\mathrm{O} / \mathrm{Fe}]$ trend is similar for all four migration prescriptions, and the model prediction is in reasonable agreement with the APOGEE measurements. From the colour-coding in the postprocessing model, one can see that the predicted relation is insensitive to the chemical evolution parameters across the range represented in our Galactic radial zones, differing only in the precise value of $[\mathrm{O} / \mathrm{Fe}]$ reached at late times. Nonetheless, these differences are large enough that radial mixing causes a large spread in age at low values of $[\mathrm{O} / \mathrm{Fe}]$, where the median trend itself becomes shallow. Nearly all high $[\mathrm{O} / \mathrm{Fe}]$ stars are old. The spread is similar, but not identical, among the four migration prescriptions, and it is in reasonable agreement with the observationally inferred spread. This agreement is a significant suc-

4 In order to avoid confusion, we distinguish between solar "annulus" and solar "neighbourhood" by defining the solar neighbourhood to be the solar annulus population at $|z| \leq 0.5 \mathrm{kpc}$. This definition should approximate a sample within a spherical radius of $\sim 0.5 \mathrm{kpc}$ around the Sun, similar to typical observational definitions. cess of the migration predicted by the h277 simulation in concert with our GCE model.

Although their median trends and characteristic spreads are similar, the diffusion and linear migration models show a marked diffrence from the post-processing and sudden models, predicting populations of young ( $\$ 4 \mathrm{Gyr})$ and intermediate age $(4-7 \mathrm{Gyr}) \alpha$-enhanced stars $([\mathrm{O} / \mathrm{Fe}] \approx+0.1-0.2)$ in the solar neighbourhood, which formed at large $R_{\mathrm{gal}}$ with $[\mathrm{O} / \mathrm{Fe}]$ values well above the main trend for their age. They arise from the large fluctuations in the SN Ia rate at large $R_{\text {gal }}$ shown in Fig. 8 and discussed in $\S 3.1$, which occur when stellar populations migrate to different radii before producing most of their SNe Ia. When the SN Ia Fe production rate fluctuates downward, $[\mathrm{O} / \mathrm{Fe}]$ fluctuates upward in the ISM, and the stars that form from it inherit such a composition. These stars then migrate to the solar annulus. The presence of young and intermediate-age $\alpha$ enhanced stars in APOGEE has been demonstrated using ages based on carbon-to-nitrogen ratios (Martig et al. 2016) calibrated against the asteroseismic ages of the APOKASC catalog (Pinsonneault et al. 2014), and with the asteroseismic ages directly (Silva Aguirre et al. 2018).

Silva Aguirre et al. (2018) demonstrate that these stars have kinematics similar to the rest of the high- $\alpha$ population, and they suggest that they are in fact old stars "rejuvenated" by mergers or mass transfer events. In a sample of 51 young, $\alpha$-rich red giants, Hekker \& Johnson (2019) demonstrate that a portion of these stars have carbonto-nitrogen ratios consistent with mass transfer events, but that others do not, indicating that they are either truly young stars or the result of mergers on the main sequence. Weinberg et al. (2017) and Johnson $\&$ Weinberg (2020) proposed that young $\alpha$-rich stars could arise in bursts of star formation that enhance the rate of CCSN enrichment. The explanation suggested here is in some sense the opposite: the stars are not so much $\alpha$-rich as they are Fe-poor because of the deficit in $\mathrm{SNe}$ Ia events. In our diffusion model, $\sim 0.2 \%$ of solar neighbourhood stars with $0.1 \leq[\mathrm{O} / \mathrm{Fe}] \leq 0.2$ have ages below $4 \mathrm{Gyr}$, but $\sim 13.9 \%$ have ages below $7 \mathrm{Gyr}$.

The right panels of Fig. 13 compare the model predictions of our 

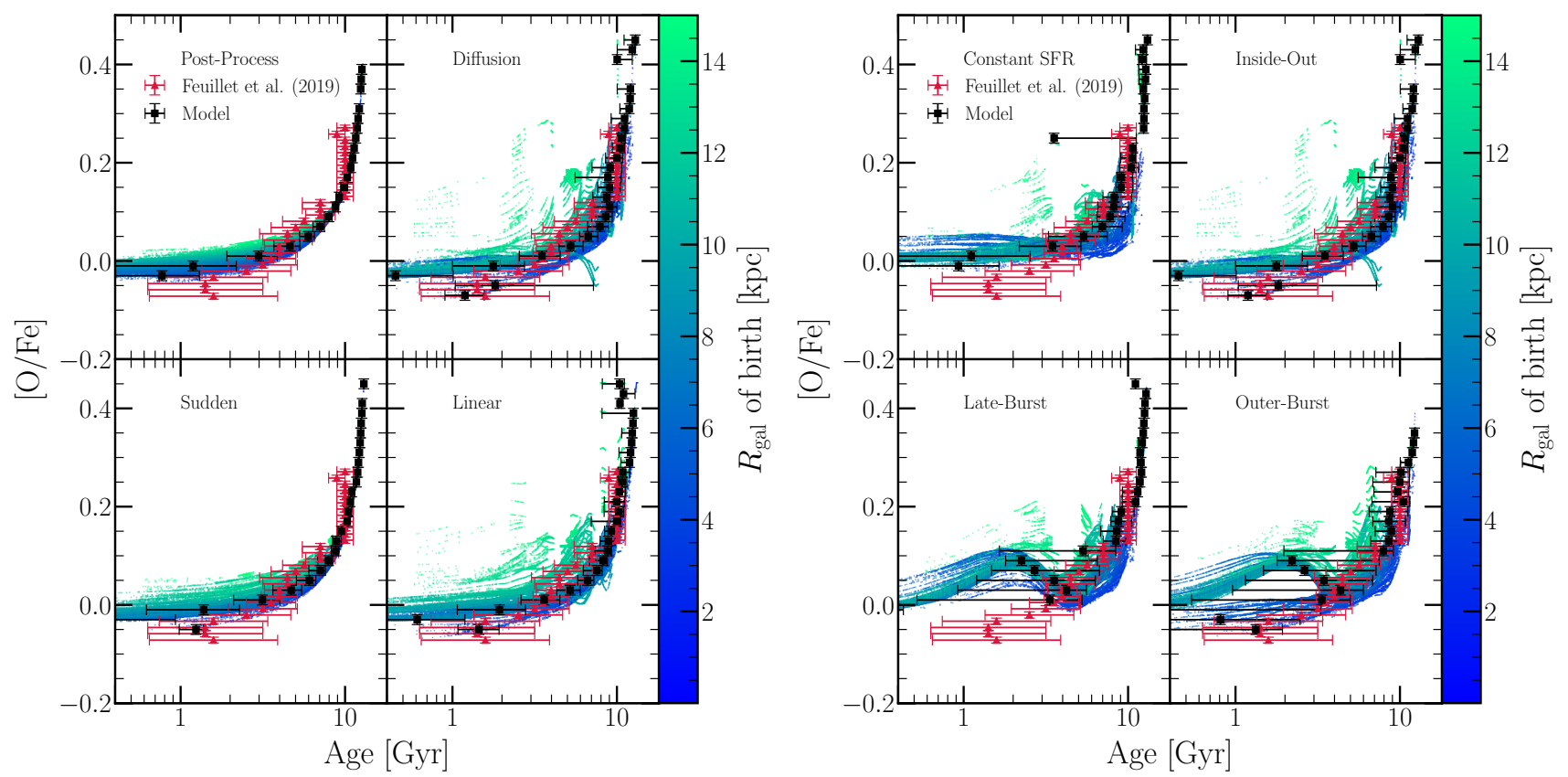

Figure 13. Left: A comparison of the predicted age- $[\mathrm{O} / \mathrm{Fe}]$ relation for the solar neighbourhood $\left(\boldsymbol{R}_{\mathrm{gal}}=7-9 \mathrm{kpc}\right.$ and $\left.|z|=0-0.5 \mathrm{kpc}\right)$ between the post-processing (upper left), diffusion (upper right), sudden (lower left), and linear (lower right) migration models, assuming our inside-out SFH. Right: The same as the left-hand panels, instead comparing the impact of our constant (upper left), inside-out (upper right), late-burst (lower left), and outer-burst (lower right) SFHs, assuming diffusion migration. In all panels, red triangles and error bars denote the observed median age and dispersion thereof in bins of $[\mathrm{O} / \mathrm{Fe}]$ as reported by Feuillet et al. (2019); here we include only their bins containing at least 15 stars. Black squares denote the mass-weighted median age in $0.02-\mathrm{dex}$ bins in [O/Fe] predicted by our models, with error bars denoting the 16th and 84th percentiles of the mass-weighted age distribution in those bins. Points in the background denote each individual stellar population from the model with a final position in the solar neighbourhood, colour-coded according to their Galactocentric radius of birth.

four different SFHs, all assuming the diffusion migration prescription, with the same plotting scheme and colour-coding as in the left panels. The predicted trend for the constant SFR model is similar to that of the inside-out model, but the agreement with data is slightly worse because with a constant SFR the evolutionary tracks do not extend below solar $[\mathrm{O} / \mathrm{Fe}]$. The starburst models, on the other hand, predict a $0.05-0.1$ dex upward fluctuation in $[\mathrm{O} / \mathrm{Fe}]$ at ages of $1-3 \mathrm{Gyr}$ due to the perturbed ratio of core-collapse to Type Ia supernova rates (Johnson \& Weinberg 2020). In the outer-burst model, stars formed at $R_{\mathrm{gal}}>6 \mathrm{kpc}$ follow this perturbed track while stars from the inner Galaxy follow the original track. These models are motivated by observational results which provide empirical evidence for elevated recent star formation (Mor et al. 2019; Isern 2019). However, in conjunction with our chemical evolution prescriptions they produce clear disagreement with the age-[O/Fe] distributions of Feuillet et al. (2019) and Miglio et al. (2021). This disagreement could indicate that the starbursts were more limited in Galactic radius than we have assumed, or that some other ingredient is missing from our models. The discrepancy in these panels also implies that if young $\alpha$-rich stars do arise in a starburst, then it must remain sufficiently localized that the resultant stellar populations remain outliers from an otherwise monotonic age- $[\alpha / \mathrm{Fe}]$ relation.

Fig. 14 extends the comparison of our baseline, inside-out model and the observations of Feuillet et al. (2019) to other Galactic regions. The predicted median age- $[\mathrm{O} / \mathrm{Fe}]$ trend is nearly independent of location, as we can see by comparing the black model points in each panel to the black line, which shows the solar neighbourhood prediction. Feuillet et al. (2019) report ages for $\alpha$-rich stars that are younger at large $R_{\text {gal }}$ and high $|z|$, though in most cases only by $\sim 20 \%$. Our model predicts a larger population of intermediate-age $\alpha$ rich stars at large $R_{\text {gal }}$, but this effect is not large enough to reproduce the observed trend. None of our model variants reproduce this trend of age-[O/Fe] offset with $|z|$, so if the observational result is correct then it points to a missing ingredient in the model. As with the overpredicted $[\mathrm{O} / \mathrm{Fe}]$ ratios at small $R_{\text {gal }}(\S 3.3)$, dynamical stirring of younger disc populations to higher $|z|$ by the Sagittarius dwarf could play a role in resolving this discrepancy. However, Fig. 14 shows that the model does not have a reservoir of younger high- $\alpha$ stars at low- $|z|$ available to be stirred, so some additional change would likely be needed. Furthermore, our model predicts the 16th-84th percentile ranges of the age distribution at fixed $[\mathrm{O} / \mathrm{Fe}]$ to increase for $[\mathrm{O} / \mathrm{Fe}] \gtrsim$ +0.1 stars, at least in part due to the higher frequency of young and intermediate-age $\alpha$-rich stars. This increase in the intrinsic scatter of the relation is simply a consequence of the SN Ia rate variability having a higher amplitude at large $R_{\text {gal }}$ (see discussion in $\S 3.1$ ).

In this paper, Fe is the only element that we consider with a delayed nucleosynthetic source. Nonetheless, we expect stellar migration to induce similar variability and scatter for other elements with delayed sources. Elements such as strontium ( $\mathrm{Sr}$ ) are produced by the $s$-process in AGB stars on a timescale intermediate between CCSN and SN Ia enrichment (see Fig. 5 of Johnson \& Weinberg 2020), and elements with a large contribution from low mass AGB stars could trace longer timescales. The DTD for $r$-process elements such as europium $(\mathrm{Eu})$ is uncertain because the relative importance of prompt sources (e.g., collapsars) and delayed sources (e.g., neutron star mergers) remains poorly constrained (Côté et al. 2019; Mishenina et al. 2019; Siegel 2019; Vincenzo et al. 2021b). Using hydrodynamical simulations from the Auriga project (Grand et al. 2017), van de Voort et al. (2020) indeed find that the intrinsic scatter in $r$-process abundances increases for models with longer characteristic delay times. Future comparisons of observed trends between age and abundance ratios with the predictions of models like those presented here could better isolate missing ingredients of the models, as well as potentially improving understanding of the nucleosynthetic sources.

\subsection{The Age-Metallicity Relation}

Although the AMR is usually formulated in terms of $[\mathrm{Fe} / \mathrm{H}]$, it is also interesting to quantify the age- $[\mathrm{O} / \mathrm{H}]$ relation because it is not affected by SN Ia enrichment. The extent to which the two AMRs differ indicates the extent to which the delayed timescale and impact of migration on SN Ia enrichment are important in shaping the age$[\mathrm{Fe} / \mathrm{H}]$ relation. Fig. 15 presents the age- $[\mathrm{O} / \mathrm{H}]$ relation predicted by our constant SFR model for the $|z| \leq 0.5 \mathrm{kpc}$ population at $R_{\mathrm{gal}}=$ 


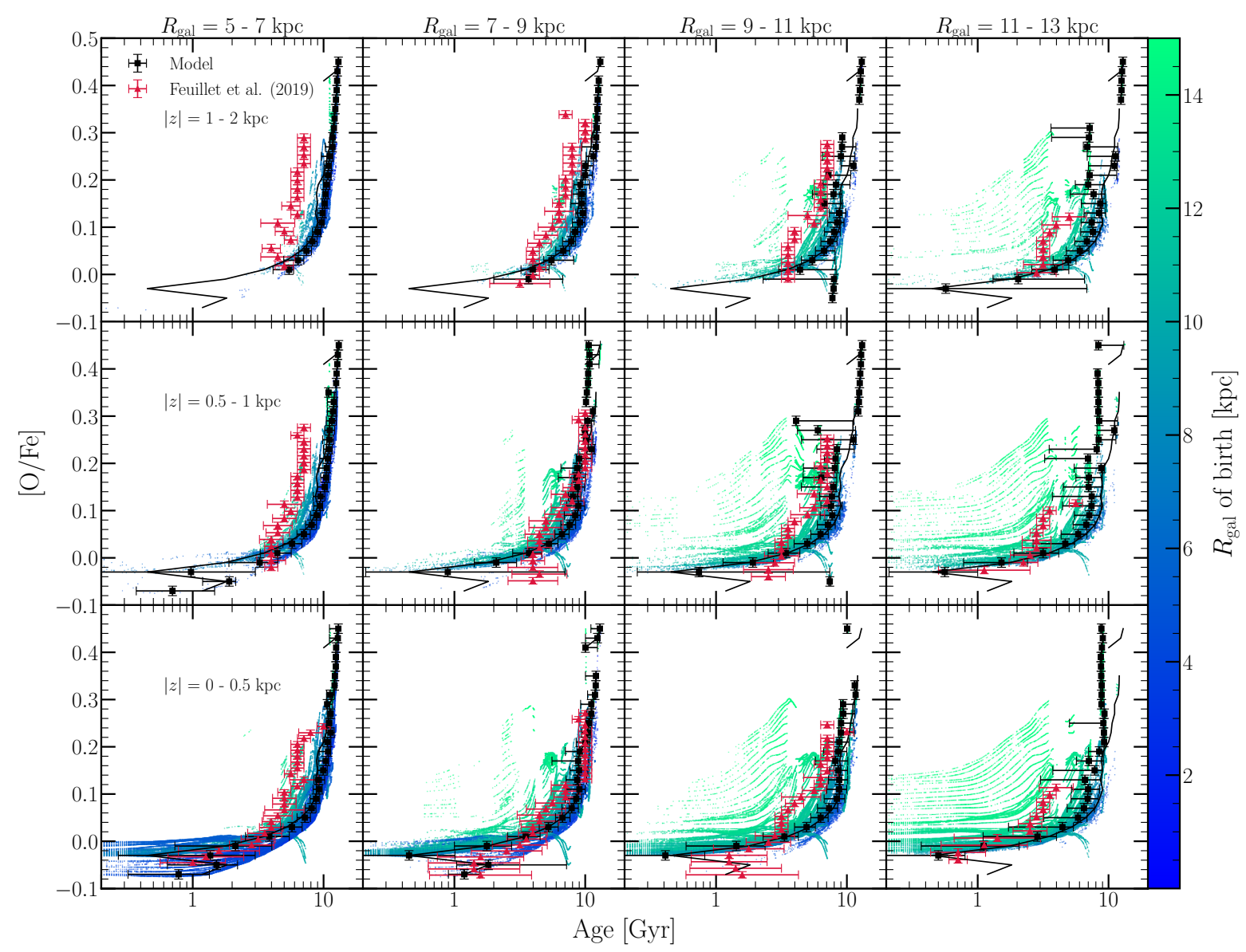

Figure 14. The age- $[\mathrm{O} / \mathrm{Fe}]$ relation in 12 Galactic regions predicted by our inside-out $\mathrm{SFH}$. Bins in Galactocentric radius are shown in columns, and labeled at the top. Bins in the disc midplane distance $|z|$ are shown in rows, noted in the left-hand column. Red triangles, black squares, error bars, and background points are as in Fig. 13 for the corresponding Galactic region. The solid black line connects the black squares in the bottom, left-middle panel, and is replicated elsewhere for reference.

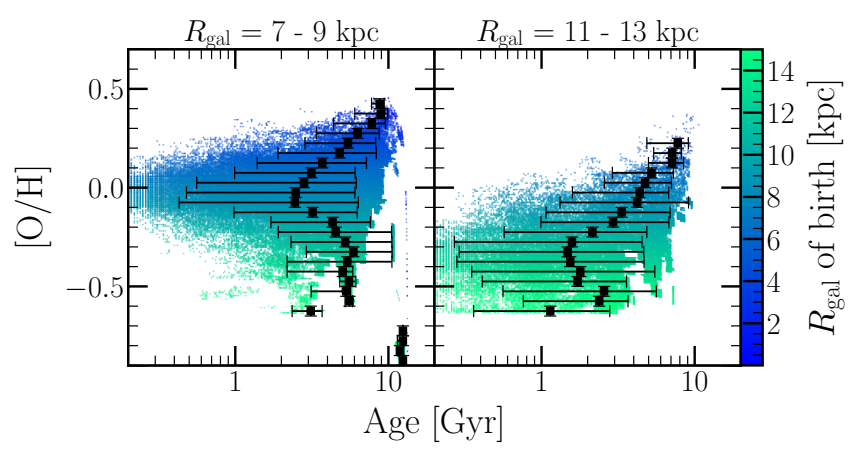

Figure 15. The age- $[\mathrm{O} / \mathrm{H}]$ relation predicted by our constant SFR model for $R_{\text {gal }}=7-9 \mathrm{kpc}$ (left) and $11-13 \mathrm{kpc}$ (right). Each panel plots only the $|z| \leq 0.5$ $\mathrm{kpc}$ population. The colored points in the background and the black squares with error bars are as in Fig. 13, but with the model prediction quantified in bins of $\Delta[\mathrm{O} / \mathrm{H}]=0.05$.

7 - 9 and $11-13 \mathrm{kpc}$. The black squares with error bars denote the mass-weighted median age as in $\S 3.4$, and we again plot the individual stellar populations in the background for reference, colour-coded according to their Galactocentric radius of birth. We omit the Feuillet et al. (2019) measurements from this figure but present observational comparisons for our more empirically motivated inside-out SFH model below. The constant SFR model allows us to isolate the effects of stellar migration from the time-dependent SFR.

The intrinsic scatter in the observed AMR has previously been interpreted as evidence for radial mixing (Edvardsson et al. 1993; Sellwood \& Binney 2002). In the solar neighbourhood, Feuillet et al. (2018) demonstrate that the most metal-rich stars tend to be signif- icantly older than solar metallicity stars. Using the Weinberg et al. (2017) analytic models of one-zone chemical evolution coupled to a simplified recipe for radial mixing, they argue that this non-monotonic trend is the result of old stars born at small $R_{\text {gal }}$ where the equilibrium abundance is high. Only the old stars are able to migrate to the solar neighbourhood due to the time required for such a change in their orbital radius.

Fig. 15 demonstrates this behaviour for our simulation-motivated radial migration prescription. Although it is observationally advantageous to measure age in bins of abundance because the abundance measurements are much more precise, it is conceptually easier to understand the model behaviour in terms of abundance spread at a given age. In both regions plotted, the youngest stars form with a composition inherited from the local ISM, which in this model is reflective of the late-time equilibrium abundance. At a given age, only stars born in a well-defined region of the Galaxy will have had adequate time to migrate to a given present-day radius. Since a range of $R_{\text {gal }}$ maps directly to a range of metallicity once the ISM is close to the equilibrium abundance, this necessitates a maximum width of the $[\mathrm{O} / \mathrm{H}]$ distribution at fixed age. With increasing age, the $[\mathrm{O} / \mathrm{H}]$ distribution then gets wider because any given present-day radius samples stars formed at a wider range of $R_{\mathrm{gal}}$. We see this effect in Fig. 15, with the colour-coding of the background points making it clear that stellar migration is the culprit. In the outer Galaxy, virtually all old stars added by migration lie above the local equilibrium metallicity. In the $R_{\text {gal }}=11-13 \mathrm{kpc}$ region, our constant SFR model predicts a nearly monotonic increase of median population age with increasing $[\mathrm{O} / \mathrm{H}]$, from $[\mathrm{O} / \mathrm{H}]=-0.5$ to +0.3 . This trend is entirely backwards from that expected by simple one-zone models of GCE.

Fig. 16 compares the age- $[\mathrm{O} / \mathrm{H}]$ (top) and age-[Fe/H] (bottom) relations predicted by our inside-out SFH model for the solar neigh- 


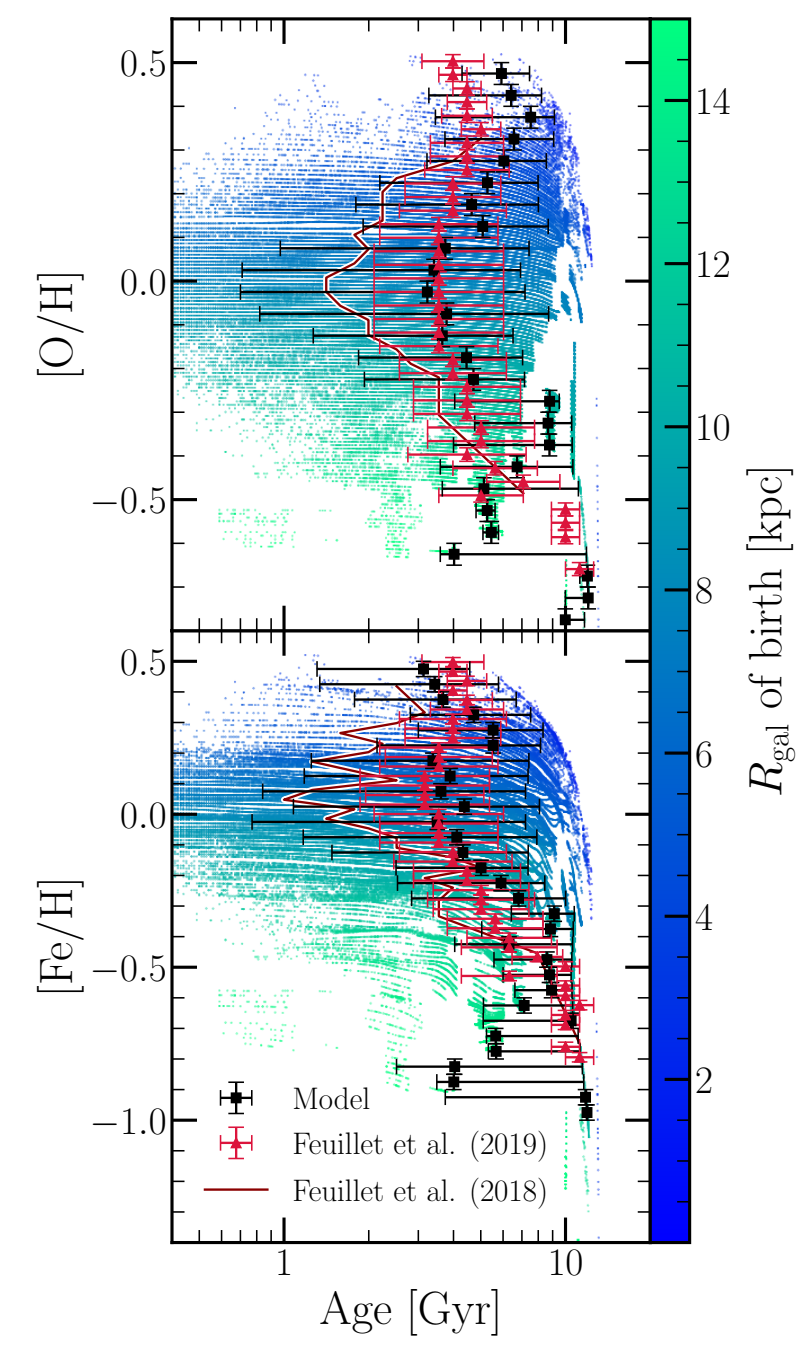

Figure 16. The age- $[\mathrm{O} / \mathrm{H}]$ (top) and age- $[\mathrm{Fe} / \mathrm{H}]$ (bottom) relations for the solar neighbourhood (i.e. $R_{\text {gal }}=7-9 \mathrm{kpc},|z| \leq 0.5 \mathrm{kpc}$ ) as predicted by the fiducial model. Red triangles, black squares, error bars, and background points are as in Fig. 13, but with the model prediction quantified in bins of $\Delta[\mathrm{O} / \mathrm{H}]=\Delta[\mathrm{Fe} / \mathrm{H}]$ $=0.05$. For comparison, we plot the Feuillet et al. (2018) measurements in a dark red line, omitting the associated uncertainties for visual clarity.

bourhood $\left(R_{\mathrm{gal}}=7-9 \mathrm{kpc}\right.$ and $\left.|z| \leq 0.5 \mathrm{kpc}\right)$ to the measurements from Feuillet et al. (2019). For reference, we add the solid dark red line, which denotes the AMR measured by Feuillet et al. (2018). Although our model shows reasonable agreement with the Feuillet et al. (2019) measurements in the solar annulus, Feuillet et al. (2018) report ages for solar metallicity that are considerably younger $(\sim 1-2 \mathrm{Gyr}$ as opposed to $\sim 3-4 \mathrm{Gyr}$ ). The origin of this difference is not clear, nor is it clear which AMR measurement is more reliable (D. Feuillet, private communication). Predictions for the inside-out SFH are similar to those shown previously for the constant SFR model, demonstrating that radial migration plays a larger role than the detailed $\mathrm{SFH}$ in determining the qualitative form of the AMR. The age- $[\mathrm{O} / \mathrm{H}]$ and age- $[\mathrm{Fe} / \mathrm{H}]$ relations are similar for both observations and models, a non-trivial result given the different time profile of SN Ia enrichment. Agreement between the model and the data is somewhat better for $[\mathrm{O} / \mathrm{H}]$ than for $[\mathrm{Fe} / \mathrm{H}]$. The model predicts a large spread in age at fixed metallicity, comparable to but somewhat larger than the spread esimated by Feuillet et al. (2019).

Fig. 17 compares the predictions of the inside-out (top) and lateburst (bottom) SFH models to the Feuillet et al. (2019) age-[Fe/H] relation in four $R_{\text {gal }}$ annuli, always with $|z| \leq 0.5 \mathrm{kpc}$. While neither model reproduces the data perfectly, the agreement is better overall for the late-burst model, a difference that is more evident from looking at all four annuli rather than the solar neighbourhood alone. The lateburst of star formation reduces the median age of $[\mathrm{Fe} / \mathrm{H}] \approx 0$ stars, typically by $1-2$ Gyr. The median age of the highest metallicity stars increases because the enhanced pristine gas accretion that fuels the starburst (see Fig. 4) dilutes the ISM metallicity, an effect that is evident in the tracks for a given birth $R_{\mathrm{gal}}$ in the lower panels. Stars from the inner Galaxy that form during the burst do so at roughly solar metallicity rather than appearing in the $[\mathrm{Fe} / \mathrm{H}]=0.2-0.5$ bins. With reduced median age at $[\mathrm{Fe} / \mathrm{H}] \approx 0$ and increased median at $[\mathrm{Fe} / \mathrm{H}]>0.2$, the late-burst model more consistently reproduces the $\mathrm{C}$-shaped AMR that is the most striking qualitative feature of the observations. The CCSN and SN Ia associated with the burst also boost the evolutionary tracks of the inner Galaxy to higher $[\mathrm{Fe} / \mathrm{H}]$ at late times, so the predicted age distribution at high $[\mathrm{Fe} / \mathrm{H}]$ is bimodal, especially at $R_{\text {gal }}=5-7 \mathrm{kpc}$. The Feuillet et al. (2019) results do not support this dichotomy, but they model the observations with the assumption of a Gaussian log age distribution, so it is worth returning to the data with the possibility of bimodal age distributions in mind.

The outer-burst model mitigates this issue since it lacks the burst at small $R_{\text {gal }}$, predicting ages for the most metal-rich stars more in line with the Feuillet et al. (2019) measurements. We illustrate this difference in Fig. 18 in Appendix A for the age-[O/H] relation. The detailed form of the AMR in our models is sensitive to the nature of the recent starburst, and its true form was likely more complicated than our parameterization. Fig. 18 also includes a comparison of the inside-out and late-burst model predictions, now without the effect of SN Ia enrichment. The late-burst model improves upon the insideout model in age- $[\mathrm{O} / \mathrm{H}]$ for the same reasons as in age- $[\mathrm{Fe} / \mathrm{H}]$, but perhaps more clearly when looking bin-by-bin.

The late-burst model is motivated by the star formation histories inferred empirically by Isern (2019) and Mor et al. (2019). Adding this late time enhancement to star formation improves agreement with the Feuillet et al. (2019) data, but it is not enough to reproduce the very young $(<2$ Gyr) median ages of solar metallicity stars found by Feuillet et al. (2018). If future analyses confirm the 2018 ages rather than the 2019 ages, they would require a later and more extreme starburst to explain them. As discussed in $\S 3.4$, the late-burst worsens agreement with the Feuillet et al. $(2018,2019)$ age- $[\mathrm{O} / \mathrm{Fe}]$ relation because of the increased $[\mathrm{O} / \mathrm{Fe}]$ at late times (see Fig. 13). We do not see an obvious way to achieve good simultaneous agreement with the observationally inferred $\mathrm{SFH}$, the age- $[\mathrm{O} / \mathrm{Fe}]$ relation, and the age- $[\mathrm{Fe} / \mathrm{H}]$ relation. Possibly a well-tuned choice of yields and the temporal and radial range of the burst could achieve such agreement; it is possible that the latter is related to the spatial dependence of the age- $[\mathrm{O} / \mathrm{Fe}]$ relation seen in the Feuillet et al. (2019) data (see Fig. 14). Alternatively, an outflow prescription that preferentially removes CCSN ejecta rather than ambient ISM (see, e.g., Vincenzo et al. 2016; Chisholm et al. 2018; Christensen et al. 2018) could mitigate the increase in $[\mathrm{O} / \mathrm{Fe}]$ during the burst (such a prescription is physically plausible, but it would require a wholesale recalibration of our model parameters). For now we conclude that radial migration can naturally explain otherwise puzzling features of the AMR and that evidence for enhanced late-time star formation in the Milky Way is ambiguous. Age-abundance relations are clearly a powerful diagnostic of GCE models, and observational investigations that demonstrate consistency across age determination methods, probe the shape of the age distribution at fixed abundance, and extend still further across the disc are highly desirable.

\section{CONCLUSIONS}

In this paper, we have modeled the Milky Way as a series of concentric rings with $\Delta R_{\text {gal }}=100 \mathrm{pc}$ width, describing each ring as a conventional one-zone chemical evolution model and allowing the exchange of stellar populations between zones to include the impact of stellar migration in the model Galaxy. Though there have been other studies that employ a similar methodology (e.g. Schönrich \& Binney 2009a,b; Kubryk et al. 2015a,b; Sharma et al. 2020), ours and the Minchev et al. $(2013,2014,2017)$ model are the only ones that make use of a hydrodynamical simulation to describe radial mixing. 


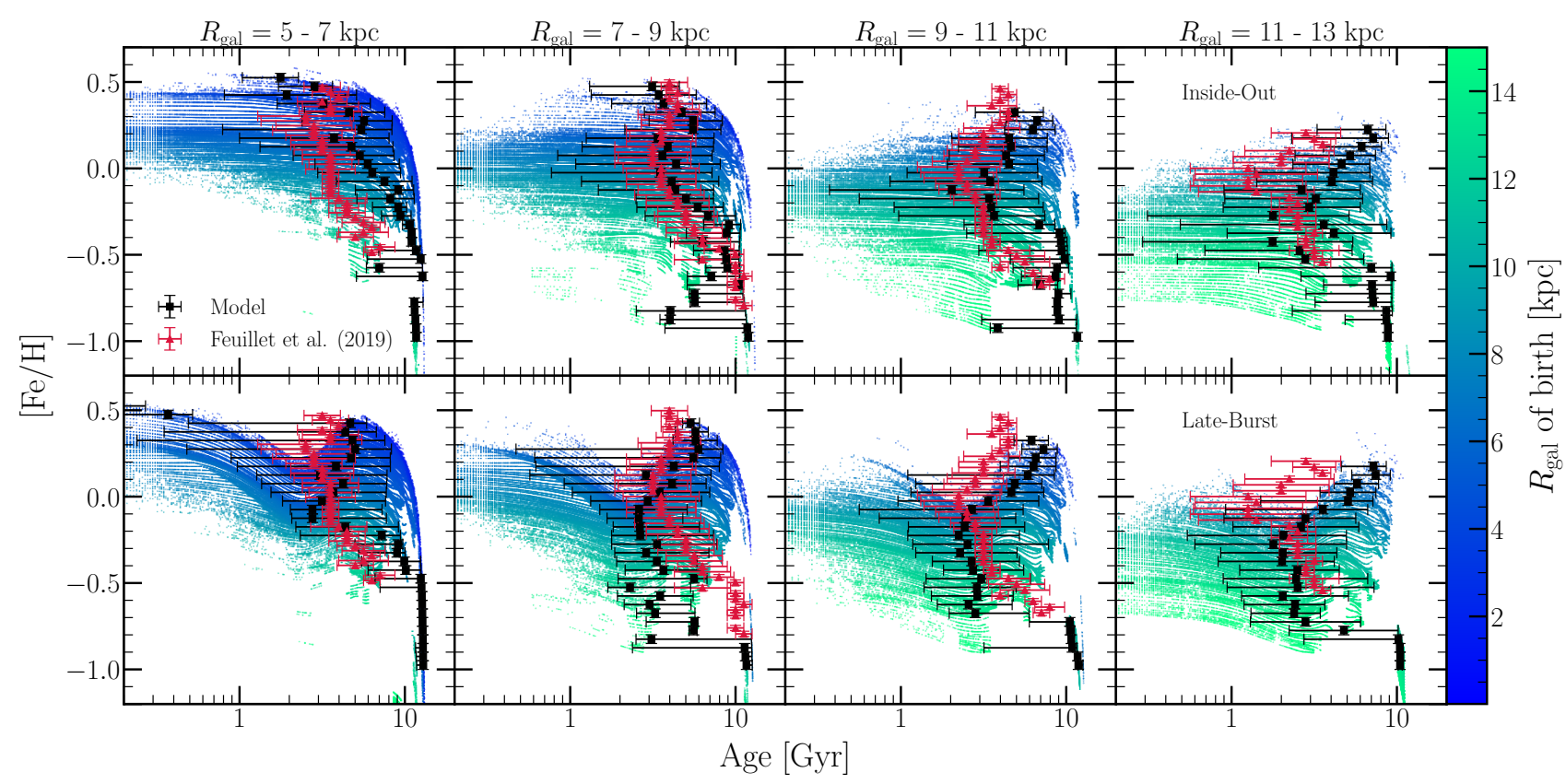

Figure 17. The age-[Fe/H] relation predicted by our inside-out (top) and late-burst (bottom) SFH models for $R_{\mathrm{gal}}=5$ - $7 \mathrm{kpc}$ (left), 7 - $9 \mathrm{kpc}$ (left middle), 9 - 11 $\mathrm{kpc}$ (right middle), and $11-13 \mathrm{kpc}$ (right). Each panel shows only the $|z| \leq 0.5 \mathrm{kpc}$ population. Red triangles, black squares, error bars, and coloured points are as in Fig. 13 for the corresponding Galactic region, but with the model prediction quantified in bins of $\Delta[\mathrm{Fe} / \mathrm{H}]=0.05$.

In our case we use the h277 zoom-in simulation of a Milky Way-like disc galaxy formed from cosmological initial conditions (Christensen et al. 2012; Zolotov et al. 2012; Loebman et al. 2012; Brooks \& Zolotov 2014; Bird et al. 2020). The simulation provides a prescription for radial migration and vertical structure with no free parameters, though there is still a choice to be made about how to model the time dependence of migration for a given stellar population (see $\S 2.2$ ). In our fiducial prescription, a stellar population traverses the $\Delta R_{\text {gal }}$ between its birth and final radius with a $\sqrt{t}$ time-dependence characteristic of diffusion, as in Frankel et al. (2018, 2020). While CCSN nucleosynthetic products are deposited instantaneously in the population's birth annulus, its SN Ia iron production follows a $t^{-1.1}$ delay-time distribution and is therefore spread across all the annuli that it traverses.

We adopt supernova yields from oxygen and iron based on a combination of theoretical and empirical constraints. We base our star formation law on the observed $\dot{\Sigma}_{\star}-\Sigma_{\mathrm{g}}$ relation in local spirals (Bigiel et al. 2010; Leroy et al. 2013; Krumholz et al. 2018), with the redshift dependence suggested by the observations of Tacconi et al. (2018). We choose a radially dependent outflow mass loading factor $\eta\left(R_{\text {gal }}\right)$ to reproduce observations of the Galactic disc metallicity gradient (e.g. Frinchaboy et al. 2013; Hayden et al. 2014; Weinberg et al. 2019). Our fiducial model adopts an inside-out SFH with efolding timescales calibrated to the Sánchez (2020) age gradients of low redshift disc galaxies (see discussion in $\S 2.5$ ). Motivated by the observational results of Mor et al. (2019) and Isern (2019), we construct models that exhibit a recent burst in star formation on top of the baseline model, as well as a constant SFH model for comparison purposes.

We have compared our fiducial inside-out SFH model and its variants to a variety of observations, most of them derived from the SDSS APOGEE survey (Majewski et al. 2017), finding a number of qualitative successes but also some significant qualitative discrepancies.

1. The relative number of high- $\alpha$ and low- $\alpha$ stars and the $[\mathrm{Fe} / \mathrm{H}]$ distribution of these two populations changes systematically with $R_{\text {gal }}$ and $|z|$, in qualitative agreement with the findings of Nidever et al. (2014) and Hayden et al. (2015). See Fig. 7.

2. The $[\mathrm{Fe} / \mathrm{H}]$ and $[\mathrm{O} / \mathrm{H}]$ distributions of stars near the Galactic plane $(|z| \leq 0.5 \mathrm{kpc})$ change shape, from negatively skewed at small $R_{\text {gal }}$ to roughly symmetric in the solar neighbourhood to pos- itively skewed in the outer Galaxy, in agreement with the findings of Hayden et al. (2015) and with our new measurements based on APOGEE DR16. The influence of radial migration on MDF shape agrees with the simplified model presented by Hayden et al. (2015) and with the numerical simulation results of Loebman et al. (2016). See Figs. 10 and 11.

3. Moving up from the midplane, the $[\mathrm{Fe} / \mathrm{H}]$ and $[\mathrm{O} / \mathrm{H}]$ MDFs become more symmetric and less dependent on $R_{\text {gal }}$, again in agreement with the observational findings of Hayden et al. (2015) and the simulation results of Loebman et al. (2016). However, the high $|z|$ MDFs are not a perfect match to the APOGEE data, especially at $|z|=0.5$ - $1 \mathrm{kpc}$ where they remain too skewed and too $R_{\mathrm{gal}}$-dependent. See Figs. 10 and 11.

4. The distributions of $[\mathrm{O} / \mathrm{Fe}]$ in bins of $[\mathrm{Fe} / \mathrm{H}]$ are broad, and their skewness and width change with $R_{\text {gal }}$ and $|z|$ in qualitative agreement with the APOGEE-based measurements of Vincenzo et al. (2021a). However, the model $[\mathrm{O} / \mathrm{Fe}]$ distributions at sub-solar $[\mathrm{Fe} / \mathrm{H}]$ do not reproduce the pronounced bimodality found by Vincenzo et al. (2021a), and the centroid of the model [O/Fe] distribution at supersolar $[\mathrm{Fe} / \mathrm{H}]$ shifts upwards with increasing $|z|$, a trend not seen in the data. See Fig. 12.

5. The trend of median stellar age in bins of $[\mathrm{O} / \mathrm{Fe}]$ agrees with the measurements of Feuillet et al. (2019) in the solar neighbourhood. The width of the $\log ($ age $)$ distribution is narrow at high $[\mathrm{O} / \mathrm{Fe}]$ and broad near solar $[\mathrm{O} / \mathrm{Fe}]$, again in agreement with the data. The model predicts a median age- $[\mathrm{O} / \mathrm{Fe}]$ relation that is nearly constant over the range $R_{\mathrm{gal}}=5-13 \mathrm{kpc}$ and $|z|=0-2 \mathrm{kpc}$, but Feuillet et al. (2019) find a $\sim 20 \%$ reduction in the median age of high $[\mathrm{O} / \mathrm{Fe}]$ stars at high $|z|$. See Figs. 13 and 14.

6. While most stars with $[\mathrm{O} / \mathrm{Fe}] \geq 0.1$ are old, the model predicts a population of young and intermediate-age $\alpha$-rich stars. These stars form in the outer Galaxy $\left(R_{\text {gal }}>10 \mathrm{kpc}\right)$ at times when the SN Ia rate, and thus the iron enrichment, has fluctuated to low values because stellar populations have migrated away before most of their SN Ia have time to explode (see Fig. 8). This mechanism, which is only realized because we track SN Ia enrichment through rings as populations migrate ( $\$ 2.2$ ), offers a novel explanation for the existence of young and intermediate-age $\alpha$-rich stars seen in APOGEE (Chiappini et al. 2015; Martig et al. 2015, 2016; Warfield et al. 2021). See Figs. 13 and 14. 
7. In the solar neighbourhood, the predicted distribution of stellar age at solar $[\mathrm{Fe} / \mathrm{H}]$ or $[\mathrm{O} / \mathrm{H}]$ is broad, and the trend of median age with metallicity is non-monotonic, with both sub-solar and super-solar metallicity stars being older on average than solar metallicity stars. These predictions agree with the observational results of Feuillet et al. (2019), though the age scatter is larger than Feuillet et al. (2019) infer, and the agreement of median trends is better for $[\mathrm{O} / \mathrm{H}]$ than for $[\mathrm{Fe} / \mathrm{H}]$. The old population at super-solar metallicity has migrated from the inner Galaxy, as suggested by Feuillet et al. (2018, 2019), and migration produces a non-monotonic age-metallicity relation throughout the disc. The agreement between predicted and observed age- $[\mathrm{Fe} / \mathrm{H}]$ realtions is noticeably worse at $R_{\text {gal }}=5-7 \mathrm{kpc}$ and somewhat worse at $R_{\text {gal }}=11-13 \mathrm{kpc}$. See Figs. $15-17$.

8. The models with late bursts of star formation, either throughout the disc or at $R_{\text {gal }}>6 \mathrm{kpc}$ only (see Fig. 4), achieve better agreement with the Feuillet et al. (2019) age-[Fe/H] relation over a range of $R_{\text {gal }}$. In particular, these models better reproduce the young median ages of solar metallicity stars and the $\mathrm{C}$-shaped form of the observed relation. However, they predict a $\sim 0.1$-dex uptick of $[\mathrm{O} / \mathrm{Fe}]$ at ages of $\sim 2 \mathrm{Gyr}$ that is not seen in the data. The SFH of these models is empirically motivated (Mor et al. 2019; Isern 2019), and we do not know if there is some variant of our implementation that would preserve their improved agreement with age- $[\mathrm{Fe} / \mathrm{H}]$ while mitigating their mismatch to age- $[\mathrm{O} / \mathrm{Fe}]$. For the other measures listed above, the predictions of these models are qualitatively similar to those of the inside-out model. See Figs. 14 and 17.

All of these predictions are affected by radial migration, and those involving vertical trends also inherit the simulation's predicted correlations between final $|z|$ and the age, birth radius, and final radius of a stellar population. We regard the overall level of agreement with many distinctive features of the Milky Way disc's abundance structure as a significant success of the models. However, at least within the framework explored here, it appears that models with smooth star formation are not able to explain the pronounced bimodality of the observed $[\alpha / \mathrm{Fe}]$ distribution. As discussed by Vincenzo et al. (2021a), we expect that this problem is generic: a one-zone model with a smooth SFH produces an $[\alpha / \mathrm{Fe}]$ distribution that peaks at low values, so it is difficult to create a superposition of such models that has a bimodal distribution.

The most widely explored solution to this problem involves a twophase SFH, with gas accretion resetting the ISM to low metallicity in between the two epochs. Versions of this scenario arise in two-infall GCE models (e.g. Chiappini et al. 1997; Spitoni et al. 2019; Khoperskov et al. 2021) and in cosmological simulations that give rise to bimodal $[\alpha / \mathrm{Fe}]$ (Mackereth et al. 2017; Grand et al. 2018; Buck 2020). An alternative scenario proposed by Clarke et al. (2019), motivated by hydrodynamic simulations, attributes the low- $\alpha$ sequence to an evolutionary track with low star formation efficiency and the high- $\alpha$ sequence to clumpy bursts of star formation that self-enrich with $\alpha$ elements. In a third, possibly fanciful scenario proposed by Weinberg et al. (2017), increased outflow efficiency at late times causes the low- $\alpha$ population to evolve "backwards" to lower $[\mathrm{Fe} / \mathrm{H}]$, after formation of the high- $\alpha$ sequence. Radial migration is likely to reshape the predictions of any of these scenarios, even if it does not fully explain bimodality on its own. These scenarios can be easily realized within our modeling framework by changing the SFH, star formation efficiency, and outflow parameterizations. We intend to explore them in future work, seeking observable signatures that can distinguish these alternative explanations for one of the most striking features of the disc abundance distribution.

The computational speed of our hybrid chemical evolution methodology makes it a valuable complement to calculating chemical evolution within full hydrodynamic cosmological simulations (e.g. Mackereth et al. 2017; Grand et al. 2018; Naiman et al. 2018; Buck 2020; Vincenzo \& Kobayashi 2020). For a given cosmological simulation, we can consider many different choices of yields and chemical evolution parameters, varying them individually to isolate physical effects and exploring parameter space to identify good fits, degeneracies, and persistent discrepancies with data. There are many obvious directions to go in extending this approach. One is to apply it to additional cosmological simulations to understand the impact of different dynamical histories, and to add the ability to model stellar populations accreted from satellites. A second is to consider additional elements that probe different nucleosynthetic pathways; many of these are already incorporated in VICE, and it is easy to add other elements and sources to models. A third is to include treatment of radial gas flows and fountains, both of which have been explored in more idealized GCE models (e.g., Lacey \& Fall 1985; Bilitewski \& Schönrich 2012; Kubryk et al. 2015a,b; Spitoni, Matteucci \& Marcon-Uchida 2013; Pezzulli \& Fraternali 2016; Sharda et al. 2021a,b). More ambitious is to implement treatment of stochastic enrichment and incomplete ISM mixing (e.g. Montes et al. 2016; Krumholz \& Ting 2018; Beniamini \& Hotokezaka 2020), which have so far been little explored in the context of Milky Way disc evolution but which are likely important in understanding the detailed correlations of elemenal abundances (Ting \& Weinberg 2021). As multi-element spectroscopic surveys grow even faster in scope and precision, efficient and flexible theoretical models will be essential for extracting the lessons they have to teach about the origin of elements and the history of the Milky Way.

\section{ACKNOWLEDGEMENTS}

We acknowledge valuable discussion with Jennifer Johnson, Adam Leroy, Grace Olivier, Amy Sardone, Jiayi Sun, Todd Thompson, and other members of the Ohio State Astronomy Gas, Galaxies, and Feedback group, as well as various participants of the SDSS Gotham 2020 virtual collaboration meeting and the virtual 236th meeting of the American Astronomical Society. We thank Diane Feuillet for providing the results of Feuillet et al. (2019) in digital form. This work was supported in part by National Science Foundation grant AST-1909841. D.H.W. is grateful for the hospitality of the Institute for Advanced Study and the support of the W.M. Keck Foundation and the Hendricks Foundation. F.V. acknowledges the support of a Fellowship from the Center for Cosmology and AstroParticle Physics at the Ohio State University.

In this paper we have made use of data from SDSS-IV APOGEE-2 DR16. Funding for the Sloan Digital Sky Survey IV has been provided by the Alfred P. Sloan Foundation, the U.S. Department of Energy Office of Science, and the Participating Institutions.

SDSS-IV acknowledges support and resources from the Center for High Performance Computing at the University of Utah. The SDSS website is www.sdss.org.

SDSS-IV is managed by the Astrophysical Research Consortium for the Participating Institutions of the SDSS Collaboration including the Brazilian Participation Group, the Carnegie Institution for Science, Carnegie Mellon University, Center for Astrophysics | Harvard \& Smithsonian, the Chilean Participation Group, the French Participation Group, Instituto de Astrofísica de Canarias, The Johns Hopkins University, Kavli Institute for the Physics and Mathematics of the Universe (IPMU) / University of Tokyo, the Korean Participation Group, Lawrence Berkeley National Laboratory, Leibniz Institut für Astrophysik Potsdam (AIP), Max-Planck-Institut für Astronomie (MPIA Heidelberg), Max-Planck-Institut für Astrophysik (MPA Garching), Max-Planck-Institut für Extraterrestrische Physik (MPE), National Astronomical Observatories of China, New Mexico State University, New York University, University of Notre Dame, Observatário Nacional / MCTI, The Ohio State University, Pennsylvania State University, Shanghai Astronomical Observatory, United Kingdom Participation Group, Universidad Nacional Autónoma de México, University of Arizona, University of Colorado Boulder, University of Oxford, University of Portsmouth, University of Utah, University of Virginia, University of Washington, University of Wisconsin, Vanderbilt University, and Yale University.

Software: Matplotlib (Hunter 2007); Astropy (Astropy Collaboration et al. 2013, 2018); NumPy (Harris et al. 2020). 


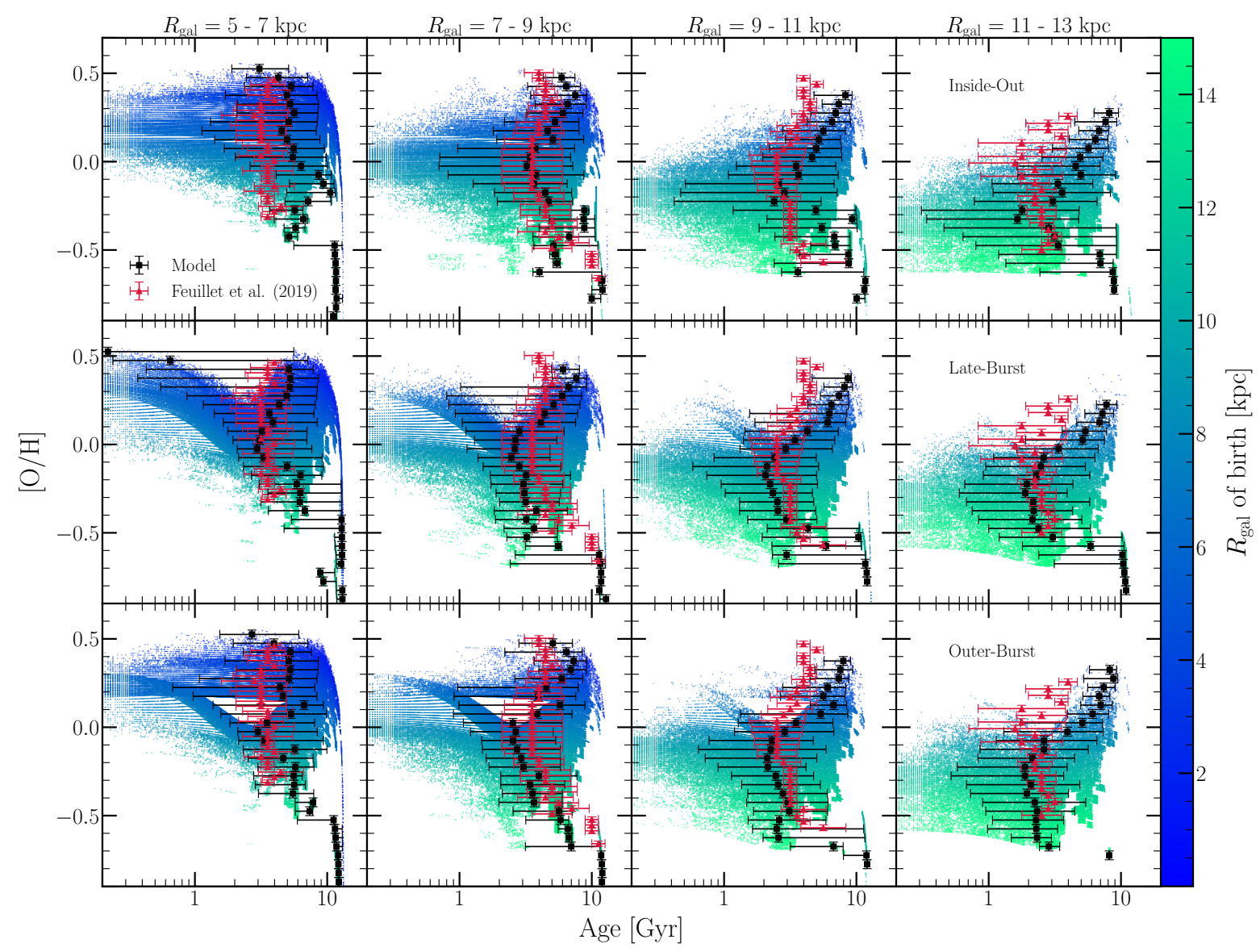

Figure 18. The same as Fig. 17, but for $[\mathrm{O} / \mathrm{H}]$, and with an additional comparison to the outer-burst model in the bottom row.

\section{DATA AVAILABILITY}

VICE is open-source software, and the code that computes abundances for our models is publicly available as well. In its GitHub repository, we provide detailed instructions on how to run our models and variations thereof; information on their computational overhead and other aspects of VICE can be found in Appendix C. All observational data appearing in this paper is publicly available, and is also included with the source code for our models and figures. Our sample of star particles from h277, including those with bulge and halo-like kinematics, is available through VICE, which will download the files automatically the first time it needs them. The rest of the data from h277 can be accessed at https://nbody.shop/data.html.

\section{Appendices}

\section{A THE AGE-[O/H] RELATION}

Fig. 18 presents a comparison of the age- $[\mathrm{O} / \mathrm{H}]$ relation predicted by our inside-out, late-burst, and outer-burst SFHs to the Feuillet et al. (2019) measurements in the same Galactic regions as in Fig. 17. The age- $[\mathrm{O} / \mathrm{H}]$ relation shows a smoother population-averaged trend than the age- $[\mathrm{Fe} / \mathrm{H}]$ relation (see Fig. 17). Affected by the variability in Type Ia supernova rates discussed in $\S 3.1$, the gas-phase Fe abundance at fixed radius fluctuates as a function of simulation time, resulting in higher intrinsic scatter in the age- $[\mathrm{Fe} / \mathrm{H}]$ relation than in age- $[\mathrm{O} / \mathrm{H}]$. We can make similar arguments about the age- $[\mathrm{O} / \mathrm{H}]$ relation as we do for age- $[\mathrm{Fe} / \mathrm{H}]$ in $\S 3.5$ : the late-burst model better reproduces the $\mathrm{C}$-shaped nature of the AMR throughout the disc, particularly beyond the solar neighbourhood. The bin-by-bin comparison is also somewhat more convincing in age $-[\mathrm{O} / \mathrm{H}]$ than in age- $[\mathrm{Fe} / \mathrm{H}]$. The late-burst model improves the agreement in all annuli with the exception of the solar annulus, where it potentially worsens the agree- ment slightly, but both models adequately reproduce the data there anyway. In comparing the late-burst model to the outer-burst model, it is clear the outer-burst model mitigates the very young ages of the most metal-rich stars at $R_{\text {gal }}=5-7 \mathrm{kpc}$ seen in the late-burst model; this is a consequence of the starburst producing a bimodal age distribution at these abundances (see discussion in $\S 3.5$ ).

\section{B NORMALIZING THE STAR FORMATION HISTORY}

In this appendix, we derive a prescription for calculating the prefactors of an adopted star formation history (SFH) for each annulus in our models. As mentioned in $\S 2.5$, this procedure requires a unitless description of the time-dependence of the SFH in each annulus, denoted $f\left(t \mid R_{\text {gal }}\right)$, and a unitless description of the radial dependence of the stellar surface density, denoted $g\left(R_{\text {gal }}\right)$. By additionally selecting a total stellar mass of the present day model Galaxy, the solution to the detailed form of the star formation history $\dot{\Sigma}_{\star}\left(t, R_{\text {gal }}\right)$ is unique. With this approach, we assume that stellar migration does not significantly impact the form of $g\left(R_{\text {gal }}\right)$, an assumption we demonstrate to be accurate in $\S 2.7$.

The surface density of star formation with units of mass per area per time can be expressed in terms of $f\left(t \mid R_{\mathrm{gal}}\right)$ as:

$\dot{\Sigma}_{\star}\left(t, R_{\text {gal }}\right)=\dot{\Sigma}_{\star}\left(t=0, R_{\text {gal }}\right) f\left(t \mid R_{\text {gal }}\right)$

and the present-day radial surface density gradient with units of mass per area as:

$\Sigma_{\star}\left(R_{\text {gal }}\right)=\Sigma_{\star}\left(R_{\text {gal }}=0\right) g\left(R_{\text {gal }}\right)$.

The integral of $\dot{\Sigma}_{\star}$ with time should yield the surface density gradient at a given radius $R_{\text {gal }}$, up to a prefactor accounting for the return of 
stellar envelopes to the interstellar medium (ISM):

$$
\begin{aligned}
\Sigma_{\star}\left(R_{\mathrm{gal}}\right) & =(1-r) \int_{0}^{T} \dot{\Sigma}_{\star}\left(t, R_{\mathrm{gal}}\right) d t \\
& =(1-r) \dot{\Sigma}_{\star}\left(t=0, R_{\mathrm{gal}}\right) \int_{0}^{T} f\left(t \mid R_{\mathrm{gal}}\right) d t \\
\dot{\Sigma}_{\star}\left(t=0, R_{\mathrm{gal}}\right) & =\Sigma_{\star}\left(R_{\mathrm{gal}}\right)\left[(1-r) \int_{0}^{T} f\left(t \mid R_{\mathrm{gal}}\right) d t\right]^{-1} \\
& =\Sigma_{\star}\left(R_{\mathrm{gal}}=0\right) g\left(R_{\mathrm{gal}}\right)\left[(1-r) \int_{0}^{T} f\left(t \mid R_{\mathrm{gal}}\right)\right]^{-1}
\end{aligned}
$$

where $(1-r) \approx 0.6$ is an adequate approximation for a Kroupa IMF (Weinberg et al. 2017, see discussion in their § 2.2). This expression relates the two unkowns introduced by equations (19) and (20). We continue by asserting that the integral of the stellar surface density over the area of the disc should be equal to the present-day stellar mass of the Milky Way:

$$
\begin{aligned}
M_{\star}^{\mathrm{MW}} & =\int_{0}^{R} \Sigma_{\star}\left(R_{\mathrm{gal}}\right) 2 \pi R_{\mathrm{gal}} d R_{\mathrm{gal}} \\
& =\Sigma_{\star}\left(R_{\mathrm{gal}}=0\right) \int_{0}^{R} g\left(R_{\mathrm{gal}}\right) 2 \pi R_{\mathrm{gal}} d R_{\mathrm{gal}} \\
\Sigma_{\star}\left(R_{\mathrm{gal}}=0\right) & =M_{\star}^{\mathrm{MW}}\left[\int_{0}^{R} g\left(R_{\mathrm{gal}}\right) 2 \pi R_{\mathrm{gal}} d R_{\mathrm{gal}}\right]^{-1} .
\end{aligned}
$$

Now plugging equation (22c) into equation (21d), and then that into equation (19) yields the desired result:

$$
\begin{aligned}
& \dot{\Sigma}_{\star}\left(t, R_{\mathrm{gal}}\right)=A f\left(t \mid R_{\mathrm{gal}}\right) g\left(R_{\mathrm{gal}}\right) \\
& A=M_{\star}^{\mathrm{MW}}\left[(1-r) \int_{0}^{R} g\left(R_{\mathrm{gal}}\right) 2 \pi R_{\mathrm{gal}} d R_{\mathrm{gal}} \int_{0}^{T} f\left(t \mid R_{\mathrm{gal}}\right) d t\right]^{-1},
\end{aligned}
$$

where the upper limits should be the maximum radius of star formation and the end time of the simulation $(15.5 \mathrm{kpc}$ and $13.2 \mathrm{Gyr}$ in this paper). This result makes intuitive sense, simply stating that the required normalization of $f\left(t \mid R_{\mathrm{gal}}\right)$ is specified by two things: the total stellar mass of the Galaxy and how steeply the stellar density falls with increasing radius. As long as the assumption that stellar migration does not significantly alter the form of $g\left(R_{\text {gal }}\right)$ is not violated, this procedure can be used to calculate prefactors for future models of disc galaxies.

\section{VICE}

VICE is an open-source python package available for Linux and Mac OS X. ${ }^{1}$ Windows users should install and use VICE entirely within the Windows Subsystem for Linux. It requires python $>=3.6$ and can be installed in a terminal via pip install vice, after which vice --docs will launch a web browser to the documentation at https://vice-astro.readthedocs.io. vice --tutorial will also launch a web browser, but to a jupyter notebook in the GitHub repository intended to familiarize first-time users with VICE's API. Python code that runs the simulations presented in this paper is included as supplementary material in the GitHub repository; they can be run from a terminal without modifying the source code. When taking into account time-dependent stellar migration, VICE requires $\sim 2.5$ CPU-hours per chemical element to compute masses, abundances, and initial and final Galactic regions for $\sim 1.6$ million stellar populations spanning a little over 1,300 timesteps. This estimate was made using a single core with a $3 \mathrm{GHz}$ processor. Although their outputs require only $\sim 235 \mathrm{MB}$ of disc space each, our models as

${ }^{1}$ Install (PyPI): https://pypi .org/project/vice Documentation: https://vice-astro.readthedocs.io Source Code: https://github.com/giganano/VICE.git computed in this paper can require up to $\sim 3 \mathrm{~GB}$ of RAM at any given time owing to the number of timesteps and stellar populations used, but these can be adjusted via command line arguments. Beyond what has been presented in this paper, VICE's capabilities include user-defined, arbitrary functions of time describing star formation and infall histories, star formation laws, outflow prescriptions, SN Ia delay-time distributions, and element-by-element infall metallicities. It allows the IMF to be a user-defined function of stellar mass. It will compute yields from supernova and asymptotic giant branch star nucleosynthesis studies, but allows the user to specify arbitrary mathematical forms for use in chemical evolution models. A complete breakdown of its abilities can be found in the documentation. While providing this level of versatility in a python package, VICE also enjoys a backend implemented in ANSI/ISO C, providing it with the powerful computing speeds of a compiled library; with typical parameters, yield calculations and one-zone model integrations require only a fraction of a second.

\section{REFERENCES}

Abolfathi B., et al., 2018, ApJS, 235, 42

Adams S. M., Kochanek C. S., Gerke J. R., Stanek K. Z., Dai X., 2017, MNRAS, 468, 4968

Adibekyan V. Z., Sousa S. G., Santos N. C., Delgado Mena E., González Hernández J. I., Israelian G., Mayor M., Khachatryan G., 2012, A\&A, 545, A32

Ahumada R., et al., 2020, ApJS, 249, 3

Andrews B. H., Weinberg D. H., Schönrich R., Johnson J. A., 2017, ApJ, 835, 224

Asplund M., Grevesse N., Sauval A. J., Scott P., 2009, ARA\&A, 47, 481

Astropy Collaboration et al., 2013, A\&A, 558, A33

Astropy Collaboration et al., 2018, AJ, 156, 123

Badenes C., et al., 2018, ApJ, 854, 147

Basinger C. M., Kochanek C. S., Adams S. M., Dai X., Stanek K. Z., 2020, arXiv e-prints, p. arXiv:2007.15658

Belfiore F., Vincenzo F., Maiolino R., Matteucci F., 2019, MNRAS, 487, 456 Beniamini P., Hotokezaka K., 2020, MNRAS, 496, 1891

Bensby T., Feltzing S., Lundström I., 2003, A\&A, 410, 527

Bigiel F., Leroy A., Walter F., Blitz L., Brinks E., de Blok W. J. G., Madore B., 2010, AJ, 140, 1194

Bilitewski T., Schönrich R., 2012, MNRAS, 426, 2266

Bird J. C., Kazantzidis S., Weinberg D. H., 2012, MNRAS, 420, 913

Bird J. C., Kazantzidis S., Weinberg D. H., Guedes J., Callegari S., Mayer L., Madau P., 2013, ApJ, 773, 43

Bird J. C., Loebman S. R., Weinberg D. H., Brooks A., Quinn T. R., Christensen C. R., 2020, arXiv e-prints, p. arXiv:2005.12948

Bland-Hawthorn J., Gerhard O., 2016, ARA\&A, 54, 529

Blanton M. R., et al., 2017, AJ, 154, 28

Bournaud F., Elmegreen B. G., 2009, ApJ, 694, L158

Bournaud F., Elmegreen B. G., Martig M., 2009, ApJ, 707, L1

Bovy J., Rix H.-W., Schlafly E. F., Nidever D. L., Holtzman J. A., Shetrone M., Beers T. C., 2016, ApJ, 823, 30

Brook C. B., et al., 2012, MNRAS, 426, 690

Brooks A. M., Zolotov A., 2014, ApJ, 786, 87

Brooks A. M., Papastergis E., Christensen C. R., Governato F., Stilp A., Quinn T. R., Wadsley J., 2017, ApJ, 850, 97

Brown J. S., et al., 2019, MNRAS, 484, 3785

Buck T., 2020, MNRAS, 491, 5435

Buck T., Obreja A., Macciò A. V., Minchev I., Dutton A. A., Ostriker J. P., 2020, MNRAS, 491, 3461

Buck T., Rybizki J., Buder S., Obreja A., Macciò A. V., Pfrommer C., Steinmetz M., Ness M., 2021, arXiv e-prints, p. arXiv:2103.03884

Bundy K., et al., 2015, ApJ, 798, 7

Chiappini C., Matteucci F., Gratton R., 1997, ApJ, 477, 765

Chiappini C., Matteucci F., Romano D., 2001, ApJ, 554, 1044

Chiappini C., et al., 2015, A\&A, 576, L12

Chieffi A., Limongi M., 2004, ApJ, 608, 405

Chieffi A., Limongi M., 2013, ApJ, 764, 21

Chisholm J., Tremonti C., Leitherer C., 2018, MNRAS, 481, 1690

Christensen C., Quinn T., Governato F., Stilp A., Shen S., Wadsley J., 2012, MNRAS, 425, 3058

Christensen C. R., Brooks A. M., Fisher D. B., Governato F., McCleary J., Quinn T. R., Shen S., Wadsley J., 2014a, MNRAS, 440, L51 
Christensen C. R., Governato F., Quinn T., Brooks A. M., Shen S., McCleary J., Fisher D. B., Wadsley J., 2014b, MNRAS, 440, 2843

Christensen C. R., Davé R., Governato F., Pontzen A., Brooks A., Munshi F., Quinn T., Wadsley J., 2016, ApJ, 824, 57

Christensen C. R., Davé R., Brooks A., Quinn T., Shen S., 2018, ApJ, 867, 142

Clarke A. J., et al., 2019, MNRAS, 484, 3476

Côté B., et al., 2019, ApJ, 875, 106

Cristallo S., et al., 2011, ApJS, 197, 17

de los Reyes M. A. C., Kennicutt Robert C. J., 2019, ApJ, 872, 16

Duong L., Asplund M., Nataf D. M., Freeman K. C., Ness M., Howes L. M., 2019, MNRAS, 486, 3586

Edvardsson B., Andersen J., Gustafsson B., Lambert D. L., Nissen P. E., Tomkin J., 1993, A\&A, 500, 391

Eisenstein D. J., et al., 2011, AJ, 142, 72

Ellison S. L., Lin L., Thorp M. D., Pan H.-A., Scudder J. M., Sánchez S. F., Bluck A. F. L., Maiolino R., 2021, MNRAS, 501, 4777

Ertl T., Janka H. T., Woosley S. E., Sukhbold T., Ugliano M., 2016, ApJ, 818, 124

Feuillet D. K., et al., 2018, MNRAS, 477, 2326

Feuillet D. K., Frankel N., Lind K., Frinchaboy P. M., García-Hernández D. A., Lane R. R., Nitschelm C., Roman-Lopes A. r., 2019, MNRAS, 489, 1742

Forbes J., Krumholz M., Burkert A., 2012, ApJ, 754, 48

Frankel N., Rix H.-W., Ting Y.-S., Ness M., Hogg D. W., 2018, ApJ, 865, 96

Frankel N., Sanders J., Rix H.-W., Ting Y.-S., Ness M., 2019, ApJ, 884, 99

Frankel N., Sanders J., Ting Y.-S., Rix H.-W., 2020, ApJ, 896, 15

Fraternali F., Tomassetti M., 2012, MNRAS, 426, 2166

Freudenburg J. K. C., Weinberg D. H., Hayden M. R., Holtzman J. A., 2017, ApJ, 849, 17

Frinchaboy P. M., et al., 2013, ApJ, 777, L1

Fuhrmann K., 1998, A\&A, 338, 161

Gaia Collaboration et al., 2018, A\&A, 616, A1

García-Benito R., et al., 2017, A\&A, 608, A27

García Pérez A. E., et al., 2016, AJ, 151, 144

Gerke J. R., Kochanek C. S., Stanek K. Z., 2015, MNRAS, 450, 3289

González Delgado R. M., et al., 2014, A\&A, 562, A47

Governato F., et al., 2012, MNRAS, 422, 1231

Grand R. J. J., Kawata D., Cropper M., 2012a, MNRAS, 421, 1529

Grand R. J. J., Kawata D., Cropper M., 2012b, MNRAS, 421, 1529

Grand R. J. J., et al., 2017, MNRAS, 467, 179

Grand R. J. J., et al., 2018, MNRAS, 474, 3629

Griffith E., et al., 2021, ApJ, 909, 77

Grisoni V., Spitoni E., Matteucci F., Recio-Blanco A., de Laverny P., Hayden M., Mikolaitis S., Worley C. C., 2017, MNRAS, 472, 3637

Harris C. R., et al., 2020, Nature, 585, 357

Hayden M. R., et al., 2014, AJ, 147, 116

Hayden M. R., et al., 2015, ApJ, 808, 132

Haywood M., Lehnert M. D., Di Matteo P., Snaith O., Schultheis M., Katz D., Gómez A., 2016, A\&A, 589, A66

Hekker S., Johnson J. A., 2019, MNRAS, 487, 4343

Holoien T. W. S., et al., 2019, MNRAS, 484, 1899

Holtzman J. A., et al., 2015, AJ, 150, 148

Hunter J. D., 2007, Computing in Science \& Engineering, 9, 90

Isern J., 2019, ApJ, 878, L11

Izzard R. G., Preece H., Jofre P., Halabi G. M., Masseron T., Tout C. A., 2018, MNRAS, 473, 2984

Jenkins A., 1992, MNRAS, 257, 620

Jenkins A., Binney J., 1990, MNRAS, 245, 305

Johnson J. A., 2019, Science, 363, 474

Johnson J. W., Weinberg D. H., 2020, MNRAS, 498, 1364

Jönsson H., et al., 2020, AJ, 160, 120

Kalberla P. M. W., Kerp J., 2009, ARA\&A, 47, 27

Kalirai J. S., Hansen B. M. S., Kelson D. D., Reitzel D. B., Rich R. M., Richer H. B., 2008, ApJ, 676, 594

Kennicutt Robert C. J., 1998, ApJ, 498, 541

Kennicutt R. C., Evans N. J., 2012, ARA\&A, 50, 531

Kennicutt Robert C. J., de los Reyes M. A. C., 2021, ApJ, 908, 61

Khoperskov S., Haywood M., Snaith O., Di Matteo P., Lehnert M., Vasiliev E., Naroenkov S., Berczik P., 2021, MNRAS, 501, 5176

Kroupa P., 2001, MNRAS, 322, 231

Krumholz M. R., Ting Y.-S., 2018, MNRAS, 475, 2236

Krumholz M. R., Burkhart B., Forbes J. C., Crocker R. M., 2018, MNRAS, 477, 2716

Kubryk M., Prantzos N., Athanassoula E., 2013, MNRAS, 436, 1479
Kubryk M., Prantzos N., Athanassoula E., 2015a, A\&A, 580, A126

Kubryk M., Prantzos N., Athanassoula E., 2015b, A\&A, 580, A127

Lacey C. G., Fall S. M., 1985, ApJ, 290, 154

Law D. R., Majewski S. R., 2010, ApJ, 714, 229

Leroy A. K., Walter F., Brinks E., Bigiel F., de Blok W. J. G., Madore B., Thornley M. D., 2008, AJ, 136, 2782

Leroy A. K., et al., 2013, AJ, 146, 19

Lian J., et al., 2020a, MNRAS, 494, 2561

Lian J., et al., 2020b, MNRAS, 497, 2371

Licquia T. C., Newman J. A., 2015, ApJ, 806, 96

Liu L., Gao Y., Greve T. R., 2015, ApJ, 805, 31

Loebman S. R., Roškar R., Debattista V. P., Ivezić Ž., Quinn T. R., Wadsley J., 2011, ApJ, 737, 8

Loebman S. R., Ivezić Ž., Quinn T. R., Governato F., Brooks A. M., Christensen C. R., Jurić M., 2012, ApJ, 758, L23

Loebman S. R., et al., 2014, ApJ, 794, 151

Loebman S. R., Debattista V. P., Nidever D. L., Hayden M. R., Holtzman J. A., Clarke A. J., Roškar R., Valluri M., 2016, ApJ, 818, L6

Lu Y., Ness M., Buck T., Zinn J., 2021, arXiv e-prints, p. arXiv:2102.12003

Mackereth J. T., et al., 2017, MNRAS, 471, 3057

Madau P., Dickinson M., 2014, ARA\&A, 52, 415

Majewski S. R., et al., 2017, AJ, 154, 94

Maoz D., Graur O., 2017, ApJ, 848, 25

Maoz D., Mannucci F., 2012, Publ. Astron. Soc. Australia, 29, 447

Martig M., Bournaud F., Croton D. J., Dekel A., Teyssier R., 2012, ApJ, 756, 26

Martig M., et al., 2015, MNRAS, 451, 2230

Martig M., et al., 2016, MNRAS, 456, 3655

Matteucci F., Francois P., 1989, MNRAS, 239, 885

Miglio A., et al., 2021, A\&A, 645, A85

Mihalas D., Binney J., 1981, Galactic astronomy. Structure and kinematics

Minchev I., Famaey B., Combes F., Di Matteo P., Mouhcine M., Wozniak H., 2011, A\&A, 527, A147

Minchev I., Famaey B., Quillen A. C., Dehnen W., Martig M., Siebert A., 2012, A\&A, 548, A127

Minchev I., Chiappini C., Martig M., 2013, A\&A, 558, A9

Minchev I., Chiappini C., Martig M., 2014, A\&A, 572, A92

Minchev I., Steinmetz M., Chiappini C., Martig M., Anders F., Matijevic G., de Jong R. S., 2017, ApJ, 834, 27

Mishenina T., Pignatari M., Gorbaneva T., Bisterzo S., Travaglio C., Thielemann F. K., Soubiran C., 2019, MNRAS, 484, 3846

Moe M., Kratter K. M., Badenes C., 2019, ApJ, 875, 61

Montes G., Ramirez-Ruiz E., Naiman J., Shen S., Lee W. H., 2016, ApJ, 830, 12

Mor R., Robin A. C., Figueras F., Roca-Fàbrega S., Luri X., 2019, A\&A, 624, L1

Munshi F., et al., 2013, ApJ, 766, 56

Naiman J. P., et al., 2018, MNRAS, 477, 1206

Nidever D. L., et al., 2014, ApJ, 796, 38

Noguchi M., 2018, Nature, 559, 585

Palla M., Matteucci F., Spitoni E., Vincenzo F., Grisoni V., 2020, MNRAS, 498, 1710

Peek J. E. G., 2009, ApJ, 698, 1429

Pejcha O., Thompson T. A., 2015, ApJ, 801, 90

Pezzulli G., Fraternali F., 2016, MNRAS, 455, 2308

Pinsonneault M. H., et al., 2014, ApJS, 215, 19

Pontzen A., Roškar R., Stinson G., Woods R., 2013, pynbody: N-Body/SPH analysis for python (ascl:1305.002)

Prantzos N., Aubert O., 1995, A\&A, 302, 69

Radburn-Smith D. J., et al., 2012, ApJ, 753, 138

Ramírez I., Allende Prieto C., Lambert D. L., 2013, ApJ, 764, 78

Romano D., Karakas A. I., Tosi M., Matteucci F., 2010, A\&A, 522, A32

Roškar R., Debattista V. P., Stinson G. S., Quinn T. R., Kaufmann T., Wadsley J., 2008a, ApJ, 675, L65

Roškar R., Debattista V. P., Quinn T. R., Stinson G. S., Wadsley J., 2008b, ApJ, 684, L79

Ruiz-Lara T., Gallart C., Bernard E. J., Cassisi S., 2020, Nature Astronomy, 4, 965

Sánchez S. F., 2020, ARA\&A, 58, 99

Schönrich R., Binney J., 2009a, MNRAS, 396, 203

Schönrich R., Binney J., 2009b, MNRAS, 399, 1145

Sellwood J. A., Binney J. J., 2002, MNRAS, 336, 785

Sharda P., Krumholz M. R., Wisnioski E., Acharyya A., Federrath C., Forbes J. C., 2021a, arXiv e-prints, p. arXiv:2102.09733 


\section{J.W. Johnson et al.}

Sharda P., Krumholz M. R., Wisnioski E., Forbes J. C., Federrath C., Acharyya A., 2021b, MNRAS, 502, 5935

Sharma S., Hayden M. R., Bland-Hawthorn J., 2020, arXiv e-prints, p. arXiv:2005.03646

Siegel D. M., 2019, European Physical Journal A, 55, 203

Silva Aguirre V., et al., 2018, MNRAS, 475, 5487

Solway M., Sellwood J. A., Schönrich R., 2012, MNRAS, 422, 1363

Spitoni E., Matteucci F., Marcon-Uchida M. M., 2013, A\&A, 551, A123

Spitoni E., Vincenzo F., Matteucci F., Romano D., 2016, MNRAS, 458, 2541

Spitoni E., Vincenzo F., Matteucci F., 2017, A\&A, 599, A6

Spitoni E., Matteucci F., Jönsson H., Ryde N., Romano D., 2018, A\&A, 612, A16

Spitoni E., Silva Aguirre V., Matteucci F., Calura F., Grisoni V., 2019, A\&A, 623, A60

Spitoni E., Verma K., Silva Aguirre V., Calura F., 2020, A\&A, 635, A58

Spitoni E., et al., 2021, A\&A, 647, A73

Sukhbold T., Ertl T., Woosley S. E., Brown J. M., Janka H. T., 2016, ApJ, 821,38

Sysoliatina K., Just A., 2021, A\&A, 647, A39

Tacconi L. J., et al., 2018, ApJ, 853, 179

Ting Y.-S., Weinberg D. H., 2021, arXiv e-prints, p. arXiv:2102.04992

van de Voort F., Pakmor R., Grand R. J. J., Springel V., Gómez F. A., Marinacci F., 2020, MNRAS, 494, 4867

Vincenzo F., Kobayashi C., 2020, MNRAS, 496, 80

Vincenzo F., Belfiore F., Maiolino R., Matteucci F., Ventura P., 2016, MNRAS, 458,3466

Vincenzo F., Kobayashi C., Yuan T., 2019, MNRAS, 488, 4674

Vincenzo F., Weinberg D. H., Miglio A., Lane R. R., Roman-Lopes A., 2021a, arXiv e-prints, p. arXiv:2101.04488

Vincenzo F., Thompson T. A., Weinberg D. H., Griffith E. J., Johnson J. W., Johnson J. A., 2021b, arXiv e-prints, p. arXiv:2102.04920

Wadsley J. W., Stadel J., Quinn T., 2004, New Astron., 9, 137

Warfield J. T., et al., 2021, AJ, 161, 100

Weinberg D. H., Andrews B. H., Freudenburg J., 2017, ApJ, 837, 183

Weinberg D. H., et al., 2019, ApJ, 874, 102

Wielen R., Fuchs B., Dettbarn C., 1996, A\&A, 314, 438

Wyse R. F. G., Silk J., 1989, ApJ, 339, 700

Yu S., et al., 2021, arXiv e-prints, p. arXiv:2103.03888

Zolotov A., et al., 2012, ApJ, 761, 71 